Pontifícia Universidade Católica $_{\text {a }}$

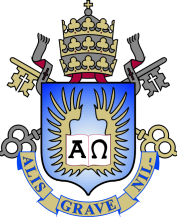

Guilherme Brandão Guglielmo

Cohomologia de Fibrados Flag Homogêneos

Dissertação de Mestrado

Dissertação apresentada como requisito parcial para obtenção do grau de Mestre pelo Programa de Pós-graduação em Matemática, do Departamento de Matemática da PUC-Rio.

Orientador: Prof. David Francisco Martínez Torres 


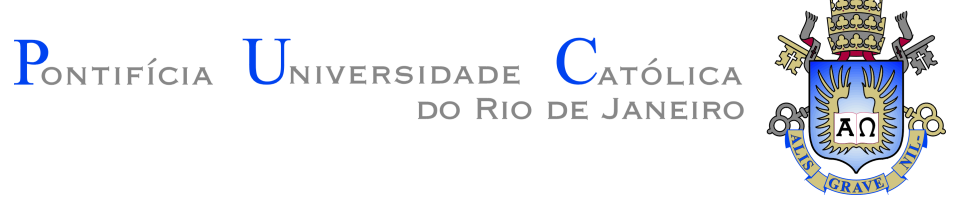

Guilherme Brandão Guglielmo

\title{
Cohomologia de Fibrados Flag Homogêneos
}

Dissertação apresentada como requisito parcial para obtenção do grau de Mestre pelo Programa de Pós-graduação em Matemática da PUC-Rio. Aprovada pela Comissão Examinadora abaixo:

\author{
Prof. David Francisco Martínez Torres \\ Orientador \\ Departamento de Matemática - PUC-Rio
}

Prof. Carlos Tomei

Departamento de Matemática - PUC-Rio

Prof. Ivan Struchiner

Departamento de Matemática - USP

Prof. María Amelia Salazar Pinzón

Departamento de Matemática Aplicada - UFF

Rio de Janeiro, 26 de Fevereiro de 2021 
Todos os direitos reservados. A reprodução, total ou parcial do trabalho, é proibida sem a autorização da universidade, do autor e do orientador.

Guilherme Brandão Guglielmo

Graduou-se em licenciatura em Matemática pela Universidade Federal Fluminense (Rio de Janeiro, Brasil)

Ficha Catalográfica

GUGLIELMO, G. B.

Cohomologia de Fibrados Flag Homogêneos / Guilherme Brandão Guglielmo; orientador: David Francisco Martínez Torres. - 2021.

146 f: il. color. ; $30 \mathrm{~cm}$

Dissertação (mestrado) - Pontifícia Universidade Católica do Rio de Janeiro, Departamento de Matemática, 2021.

Inclui bibliografia

1. Matemática - Teses. 2. Geometria, Topologia - Teses. 3. Fibrado flag homogêneo. 4. Cohomologia equivariante. 5. $G^{*}$-módulo. 6. Modelo de Cartan. I. Martínez Torres, David.. II. Pontifícia Universidade Católica do Rio de Janeiro. Departamento de Matemática. III. Título. 


\section{Agradecimentos}

Ao meu orientador Professor David Martínez Torres pela ajuda, paciência estímulo e parceria sem as quais esta dissertação não seria possível.

À banca por aceitarem avaliar essa dissertação.

À minha esposa Carla pelo apoio durante toda a dissetação.

À minha família, pela educação, apoio e compreenção.

Ao meu amigo e padrinho João pelo apoio moral.

Aos meus amigos da PUC-Rio.

Ao CNPq, à Petrobrás e à PUC-Rio, pelos auxílios concedidos, sem os quais este trabalho não poderia ter sido realizado.

O presente trabalho foi realizado com apoio da Coordenação de Aperfeiçoamento de Pessoal de Nível Superior-Brasil (Capes)- Código do Financiamento 001. 


\section{Resumo}

GUGlielmO, G. B.; Martínez Torres, David.. Cohomologia de Fibrados Flag Homogêneos. Rio de Janeiro, 2021. 146p. Dissertação de Mestrado - Departamento de Matemática, Pontifícia Universidade Católica do Rio de Janeiro.

Esta dissertação tem como objetivo exibir uma fórmula para cálcular o anel de cohomologia de um fibrado flag homogêneo de um grupo de Lie $G$ compacto e conexo. Para concluir o resultado é usado a cohomologia equivariante, em particular, sua abordagem mais algébrica. Isto implica introduzir $G^{*}$ módulos e sua teoria equivariante, o que passa também por introduzir a álgebra de Weil, o modelo de Cartan e o homomorfismo característico. A demonstração do resultado também está fortemente baseada nas propriedades algébricas dos toros maximais de $G$.

\section{Palavras-chave}

Fibrado flag homogêneo; Cohomologia equivariante; $G^{*}$-módulo; Modelo de Cartan. 


\section{Abstract}

GUGLielmO, G. B.; Martínez Torres, David. (Advisor). Cohomology of Homogeneous Flag Bundles. Rio de Janeiro, 2021. 146p. Dissertação de Mestrado - Departamento de Matemática, Pontifícia Universidade Católica do Rio de Janeiro.

The purpose of this dissertation is to present a formula for calculating the cohomology ring of a homogeneous flag bundles of a compact and connected Lie $G$ group. To conclude the result, the equivalent cohomology is used, in particular, its more algebraic approach. This implies introducing $G^{*}$ modules and their equivalent theory, which also involves introducing Weil algebra, Cartan's model and characteristic homomorphism. The income statement is also strongly based on the algebraic properties of the maximal torus of $G$.

\section{Keywords}

Homogeneous flag bundle; Equivalent cohomology; $G^{*}$ module; Cartan model. 


\section{Sumário}

$\begin{array}{llr}1 & \text { Introdução } & 9\end{array}$

2 Preliminares $\quad 20$

2.1 Cohomologia de de Rham 20

2.2 Grupos de Lie e Fibrado Principal 23

2.3 Fibrado Associado e Fibrado Flag Homogêneo 30

3 Cohomologia de Fibrados $\quad 34$

3.1 Cohomologia de Fibrados a Partir de Informações Cohomológicas da Base e da Fibra $\quad 35$

$\begin{array}{ll}3.2 & \text { Cohomologia Equivariante Topológica } \\ \end{array}$

4 Identidades Diferenciais: Ação de $G$ e Derivações em $M \quad 44$

$5 \quad G^{*}$-módulos $\quad 49$

$5.1 G^{*}$-módulos e $G^{*}$-álgebras $\quad 54$

5.2 Cohomologia e Homotopias em Cadeia 57

5.3 Ação Livre e a Condição $(\mathbf{C}) \quad 62$

5.4 O Subcomplexo Básico e a Cohomologia Equivariante de $G^{*}$-álgebras 67

6 Álgebra de Weil $\quad 71$

6.1 Álgebra de Weil $\quad 73$

6.2 W a $G^{*}$-álgebra Mais Simples e o Mapa de Chern-Weil 78

$6.3 W^{*}$-módulos $\quad 81$

7 Modelos e o isomorfismo de Mathai-Quillen 83

7.1 Isomorfismo de Mathai-Quillen $\quad 83$

$\begin{array}{lll}7.2 & \text { Modelo de Cartan } & 87\end{array}$

$\begin{array}{lll}\text { 7.3 Cohomologia Equivariante de } W^{*} \text {-módulos } & 90\end{array}$

$\begin{array}{lll}7.4 & \text { O Homomorfismo Característico } & 94\end{array}$

7.5 Cohomologia Equivariante de Espaços Homogêneos 96

8 Cohomologia do Fibrado Flag Homogêneo 99

8.1 Modelo de Cartan, um Duplo Complexo 100

8.2 $H_{G}(A)$ um $S\left(g^{*}\right)^{G}$-módulo e Morfismos de $G^{*}$-módulos 103

$\begin{array}{ll}8.3 \text { Restrição de Grupo } & 106\end{array}$

8.4 Cohomologia do Fibrado Flag Homogêneo 108

$\begin{array}{lr}\text { Apêndices } & 122\end{array}$

$\begin{array}{llr}\text { Apêndice A } & \text { Prova do Teorema 2.2.9 }\end{array}$

$\begin{array}{lll}\text { Apêndice B Teorema Equivariante de de Rham } & 126\end{array}$

$\begin{array}{lll}\text { Apêndice C } & \text { Fórmulas do Capítulo } 7 & 133\end{array}$ 
Apêndice D Sequência Espectral de Complexo Duplo

136

D.1 Construção da Sequência Espectral de Complexos Duplos

136

D.2 O Funtor Graduação

143

Referências bibliográficas 


\section{1 \\ Introdução}

A cohomologia/homologia é um invariante topológico muito importante, pois admite uma natureza algébrica, uma teoria e uma estrutura, isto é, uma axiomática, que por muitas vezes torna razoável ser calculada a cohomologia de certos espaços topológicos. Para ter uma ideia disto, a cohomologia é geralmente muito mais fácil de ser calculada do que os grupos de homotopia. Aqui, por axiomática, estamos considerando os axiomas de Eilenberg-Steenrod e o axioma de excisão talvez seja o que mais auxilia em alguns cálculos e difere do grupo fundamental.

Nesta dissertação trabalharemos, sobre tudo, com uma categoria de conjuntos topológicos específica, a de variedades diferenciáveis. Nela seus morfismos são mapas suaves. A cohomologia mais sugestiva de ser usada neste caso é a cohomologia de de Rham que, podemos ver que, é isomorfa a cohomologia singular. Para ser mais preciso, graças ao teorema de de Rham, sabemos que há um isormofismo entre a cohomologia de de Rham de uma variedade diferencíavel e sua cohomologia singular calculada sobre $\mathbb{R}$.

O resultado que estamos buscando nesta dissertação é uma fórmula para calcular o anel de cohomologia de de Rham de uma variedade diferenciável bem específica, o fibrado flag homogêneo. Mas, para isso, faremos uso de outra cohomologia, a cohomologia equivariante.

$\mathrm{Na}$ teoria de de Rham existem dois grandes resultados que nos motivam: a fórmula de Künneth e o teorema de Leray-Hirsch. Ambos resultados intimamente ligados a teoria de fibrados e que nos dão uma fórmula para se calcular a cohomologia de certos tipos de variedades.

A fórmula de Künneth é um resultado interessante para se cálcular a cohomologia de fibrados triviais. Se tomarmos como hipótese que $M$ e $F$ são variedades diferenciáveis, tendo $M$ uma cobertura finita, então

$$
H^{n}(M \times F)=\underset{p+q=n}{\bigoplus} H^{P}(M) \otimes H^{q}(F)
$$


ou simplesmente

$$
H^{*}(M \times F)=H^{*}(M) \otimes H^{*}(F) .
$$

Na verdade, a hipótese de $M$ ter boa cobertura finita pode não ser necessária, precisamos apenas de alguma hipótese de finitude. Graças a [BT], esta hipótese pode ser substituída por $F$ ter cohomologia de dimensão finita.

Já o teorema de Leray-Hirsch está mais próximo do resultado que objetivamos. A ideia é calcular a cohomologia do fibrado a partir de informações cohomológicas da base e da fibra. O teorema de Leray-Hirsch diz que se considerarmos $\pi: M \rightarrow X$ um fibrado com fibra $F$ e supormos que $X$ admite boa cobertura finita, se houver classes de cohomologia global $e_{1}, \ldots, e_{r}$ em $M$ que quando restrito a cada fibra geram livremente a cohomologia da fibra, isto é, para todo $b \in X i_{b}^{*} e_{1}, \ldots, i_{b}^{*} e_{r}$ (onde $i: \pi^{-1}(b) \rightarrow M$ é a inclusão) é uma base de $H^{*}\left(\pi^{-1}(b)\right)$, então $H^{*}(M)$ é um módulo livre sobre $H^{*}(X)$ com base $\left\{e_{1}, \ldots, e_{r}\right\}$, ou seja,

$$
H^{*}(M) \simeq H^{*}(X) \otimes \mathbb{R}\left\{e_{1}, \ldots, e_{r}\right\} \simeq H^{*}(X) \otimes H^{*}(F)
$$

O resultado que visamos é parecido com este teorema, mas que admite estrutura de álgebra, não apenas de módulo. Uma vez que no teorema de Leray-Hirsher temos $H^{*}(M)$ escrito como combinação de $\left\{e_{1}, \ldots, e_{r}\right\}$ sobre $H^{*}(X)$, buscamos neste trabalho comprender também o produto dos termos $e_{1}, \ldots, e_{r}$, ou seja, polinômios sobre $H^{*}(X)$.

Como comentado anteriormente, a ideia é calcular a cohomologia de um fibrado a partir da cohomologia da base e de informações cohomológicas da fibra, mas como faremos isto exatamente no caso do fibrado flag homogêneo? Bom, para começar, consideremos $G$ um grupo de Lie compacto e conexo e, $\rho: M \rightarrow M / G$ um fibrado principal. Seja $T$ um toro maximal de $G$, isto é, um subgrupo compacto, conexo e abeliano de $G$ que é maximal pela inclusão. Em particular, como $G$ é conexo, a álgebra de Lie de $T$ é uma subálgebra de Cartan. Além disso, temos uma ação natural de $G$ em $G / T$, portanto, temos bem definido o fibrado flag homogêneo como sendo

$$
[\rho]:(M \times G / T) / G \rightarrow M / G, \quad[\rho]([p, q]) \mapsto \rho([p]) .
$$

Contudo, ainda existe uma identificação do fibrado flag homogêneo com 
$M / T$ por meio de um difeomorfismo. Desta forma, pelo diagrama

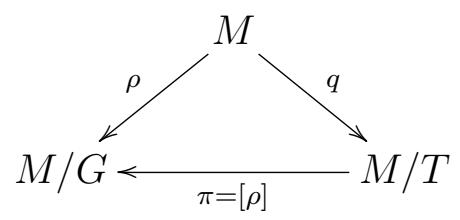

onde $\pi$ leva a órbita de $T$ na órbita de $G$ em $M$, temos que o mapa pullback $\pi^{*}$, nos garante um mapa da cohomologia de $M / G$ em $M / T$. Este mapa que nos permitirá no futuro verificar que a cohomologia de $M / T$ e, portanto, a cohomologia do fibrado flag homogêneo é

$$
H^{*}(M / G)\left[\sigma^{1}, \cdots, \sigma^{r}\right] / I
$$

onde $I$ é um ideal gerado, não diretamente, a partir da base do dual da álgebra de Lie de $T$, assim como cada variável $\sigma^{i}$, ou seja, o anel de cohomologia do fibrado flag homogêneo é um quociente de um anel de polinômios cujos coeficientes estão no anel de cohomologia da base.

Compreender cada termo da fórmula acima é bem complicado e nos dará um pouco de trabalho. Uma das dificuldades é compreender que propriedades especiais tem o toro maximal $T$ que difere de um subgrupo de Lie ordinário de $G$ que permite chegar na conclusão acima. Entretanto, mais trabalhoso e longo que isso é o processo e as discussões que nos levarão a esta fórmula. Boa parte desta dissertação tem o propósito de construir e exibir uma teoria desenvolvida inicialmente por Henri Cartan, a cohomologia equivariante, mais precisamente sua abordagem mais algébrica, a cohomologia equivariante de $G^{*}$-módulos, que nos permitirá concluir a fórmula para a cohomologia de fibrados flag homogêneos.

Mas o que seria $G^{*}$-módulo? Antes de responder esta pergunta é interessante explicar o porquê de expressarmos cohomologia equivariante de $G^{*}$ módulos e não simplesmente cohomologia equivariante. O motivo é que, como veremos nesta dissertação, podemos considerar outra forma de cohomologia equivariante. Vale ressaltar que, sobre certas condições, ambas as cohomologias equivariantes são equivalentes. Este é o teorema equivariante de de Rham e pode ser encontrado no apêndice deste trabalho. Iremos nomear esta outra forma de cohomologia equivariante como sendo a cohomologia equivariante topológica, já que, sua construção depende apenas de estruturas topológicas e variedades diferenciáveis. Esta cohomologia é um pouco mais simples de 
ser construída. A partir de um grupo de Lie $G$ compacto que age livremente em uma variedade $M$, na cohomologia equivariante topológica, denotada $H_{G}^{*}$, espera-se que definamos $H_{G}^{*}(M)$ como

$$
H^{*}(M / G)
$$

o que tem sentido já que $M / G$ é uma variedade, por $G$ agir livremente. Porém, queremos englobar na definição não apenas variedades em que $G$ aja livremente. Desta forma, para generalizar esta ideia, consideramos $E$ um espaço topológico contrátil em que $G$ age livremente e assim, definimos

$$
H_{G}^{*}(M):=H^{*}((M \times E) / G) .
$$

É sempre possível encontrar $E$ que satisfaça as condições, e a construção acima não depende da escolha de $E$. Todavia, a cohomologia equivariante topológica não nos auxiliará muito para chegarmos na fórmula da cohomologia do fibrado flag homogêneo, já que, ela carece de estruturas algébricas, pois não leva em consideração as identidades geométricas/topológicas provenientes da ação de um grupo de Lie em uma variedade diferenciável. Considerando $g$ a álgebra de Lie de $G$, as identidades que estamos comentando são as equações de Weil:

$$
\begin{gathered}
\iota_{i} \iota_{j}+\iota_{j} \iota_{i}=0 \\
\mathcal{L}_{i} \iota_{j}-\iota_{j} \mathcal{L}_{i}=\iota_{[i, j]}=c_{i j}^{k} \iota_{k} \\
\mathcal{L}_{i} \mathcal{L}_{j}-\mathcal{L}_{j} \mathcal{L}_{i}=\mathcal{L}_{[i, j]}=c_{i j}^{k} \mathcal{L}_{k} \\
d \iota_{i}+\iota_{i} d=\mathcal{L}_{i} \\
d \mathcal{L}_{i}-\mathcal{L}_{i} d=0 \\
d^{2}=0 \\
\rho_{a} \circ \mathcal{L}_{i} \circ \rho_{a}^{-1}=\mathcal{L}_{\operatorname{Ad}(a) \xi_{i}} \\
\rho_{a} \circ \iota_{i} \circ \rho_{a}^{-1}=\iota_{A d(a) \xi_{i}}
\end{gathered}
$$

onde $a \in G, \rho_{a}$ é a representação de $G$ em $\Omega(M)$ induzida pela ação de $G$ em $M, d$ a derivada exterior, $A d$ a representação adjunta, $\xi_{1}, \cdots \xi_{n}$ é uma base de $g, \iota_{\xi_{i}}=\iota_{i}$ o produto interior e, por fim, para $\omega \in \Omega(M)$, $\mathcal{L}_{i} \omega=\mathcal{L}_{\xi_{i}} \omega:=\left.\frac{d}{d t}\left(\rho_{\exp t \xi_{i}} \omega\right)\right|_{t=0}$.

Então, aqui podemos voltar a pergunta: o que são $G^{*}$-módulos? $G^{*}$ módulo é, basicamente, uma estrutura algébrica definida de forma a satisfazer 
as equações acima. Para ser mais preciso, começamos primeiro com $G$ um grupo de Lie e $g$ sua álgebra de Lie. A partir de uma base $\xi_{1}, \cdots, \xi_{n}$ de $g$, podemos definir uma super álgebra de Lie $\bar{g}:=g_{-1} \oplus g_{0} \oplus g_{1}$, com $g_{-1}$ o espaco vetorial gerado por $\iota_{1}, \cdots, \iota_{n}, g_{0}$ o espaço gerado por $\mathcal{L}_{1}, \cdots, \mathcal{L}_{n}$ e $g_{1}$ o espaço gerado por $d$. O colchete é definido em termos desta base por:

$$
\begin{gathered}
{\left[\iota_{i}, \iota_{j}\right]=0} \\
{\left[\mathcal{L}_{i}, \iota_{j}\right]=c_{i j}^{k} \iota_{k}} \\
{\left[\mathcal{L}_{i}, \mathcal{L}_{j}\right]=c_{i j}^{k} \mathcal{L}_{k}} \\
{\left[d, \iota_{i}\right]=\mathcal{L}_{i}} \\
{\left[d, \mathcal{L}_{i}\right]=0} \\
{[d, d]=0 .}
\end{gathered}
$$

Com isto, definimos um $G^{*}$-módulo como um super espaço vetorial $A$ com uma representação linear de $G$ em $A$ e um homomorfismo $\bar{g} \rightarrow \operatorname{End}(A)$ que satisfaz:

$$
\begin{gathered}
\left.\frac{d}{d t} \rho(\exp t \xi)\right|_{t=0}=\mathcal{L}_{\xi} \\
\rho(a) \mathcal{L}_{\xi} \rho\left(a^{-1}\right)=\mathcal{L}_{A d_{a} \xi} \\
\rho(a) \iota_{\xi} \rho\left(a^{-1}\right)=\iota_{A d_{a} \xi} \\
\rho(a) d \rho\left(a^{-1}\right)=d .
\end{gathered}
$$

E ainda, com uma construção parecida, podemos definir uma $G^{*}$-álgebra $A$ como uma super álgebra comutativa junto com uma representação $\rho$ de $G$ como automorfismo em $A$ e uma ação de $\bar{g}$ como uma super derivação de $A$ consistente com as equações acima. Repare que uma $G^{*}$-álgebra é uma super álgebra que é um $G^{*}$-módulo em que $G$ age como um automorfismo e $\bar{g}$ como super derivação. Um exemplo que satisfaz estas condições é justamente $\Omega(M)$.

Definimos os morfismos nesta categoria como mapas lineares que comutam com as derivações e com a representação de $G$ em $A$. Além disso, $A$ sendo um $G^{*}$-módulo, ou, $G^{*}$-álgebra, admite estrutura de um complexo cocadeia com operador fronteira $d$, assim definimos a cohomologia de $A, H(A)$ como

$$
H(A, d)
$$

Não surpreendentemente, no caso de $\Omega(M)=A$, a cohomologia $H(A)$ é a mesma que a cohomologia de de Rham de $M$. 
Como comentamos anteriormente, a cohomologia equivariante de $G^{*}$ módulos (ou $G^{*}$-ágebras) nos possibilita trabalhar do ponto de vista puramente algébrico e nos permite estudar estruturas mais simples. Neste sentido é importante detectar de forma infinitesimal a condição da ação de $G$ ser livre para ver como ela age na cohomologia. Desta forma, é interessante trabalhar com uma condição um pouco mais fraca, a condição de ser localmente livre. Esta condição diz que a ação de $G$ em $M$ é localmente livre se para cada $0 \neq \xi \in g$, o campo vetorial que gera o grupo a um parâmtro $t \mapsto \exp -t \xi$ de transformações em $M$ nunca desaparece. Ou seja,

$$
\xi_{M}^{-}(x):=\left.\frac{d}{d t}((\exp -t \xi))(x)\right|_{t=0}
$$

e $\xi_{M}^{-}(x) \neq 0$ para todo $x \in M$. Isto nos permite ter formas diferenciáveis $\theta^{1}, \cdots, \theta^{n}$ a partir de uma base $\xi_{1}, \cdots, \xi_{n}$ de $g$ que satisfaz

$$
\iota_{a} \theta^{b}=\delta_{a}^{b}
$$

Estas são as conhecidas formas de conexão. Outras formas importantes são as formas horizontais que satisfazem para $a=1, \cdots, n$,

$$
\iota_{a} \omega=0 .
$$

É interessante ver que, caso $G$ seja compacto, temos uma métrica Riemanniana na qual $T^{*} M=C \oplus C^{\perp}$, onde $C^{\perp}$ representa as formas horizontais, é uma separação $G$-invariante, isto é, $C$ é $G$-invariante.

Desta forma, procurando generalizar e algebrizar este conceito, definimos a condição $(\mathbf{C})$, isto é, dizemos que uma $G^{*}$-álgebra satisfaz a condição $(\mathbf{C})$ se existem elementos $\theta^{i} \in A_{1}$ que satisfazem $\iota_{a} \theta^{b}=\delta_{a}^{b}$ e que o subespaço $C \subset A_{1}$ gerado por estes elementos é $G$-invariante. Nomearemos esses elementos $\left(\theta^{i}\right)$ 's como elementos de conexão. Vale ressaltar que se $G$ é conexo, $C$ ser $G$ invariante é equivalente a satisfazer

$$
\mathcal{L}_{a} \theta^{j}=-c_{a b}^{j} \theta^{b}
$$

Quando $G$ é compacto e age livremente em $M$ temos o $G$ fibrado principal

$$
\pi: M \rightarrow M / G
$$

O subcomplexo $\pi^{*} \Omega(M / G) \subset \Omega(M)$ é o chamado complexo de formas 
básicas. É possível ver que $\omega$ é uma forma básica se é $G$-invariante e $\iota_{a} \omega=0$. Caso $G$ seja conexo, a $G$-invariância equivale a $\mathcal{L}_{a} \omega=0$. Assim, novamente generalizando o conceito, definimos os elementos básicos de um $G^{*}$-módulo $A$, denotado por $A_{b a s}$, os elementos que satisfazem, de forma semelhante, as condições de uma forma básica. Isto nos garante uma outra cohomologia, a cohomologia básica

$$
H\left(A_{b a s}, d\right):=H_{b a s}(A)
$$

A definição de cohomologia equivariante de $G^{*}$-módulos e $G^{*}$-álgebras é semelhante a definição de cohomologia equivariante topológica. Começamos considerando $E$ uma $G^{*}$-álgebra acíclica que satisfaz a condição $(\mathbf{C})$. Definimos a cohomologia equivariante de uma $G^{*}$-álgebra ou $G^{*}$-módulo a partir do subcomplexo básico $A \otimes E$, isto é,

$$
H_{G}(A):=H_{b a s}(A \otimes E)=H\left((A \otimes E)_{b a s}, d\right) .
$$

Em geral, quando escrevemos cohomologia equivariante de $G^{*}$-módulos, estamos nos referindo tanto a cohomologia equivariante de $G^{*}$-módulos como a de $G^{*}$-álgebras, já que suas definições são semelhantes.

Para a definição estar bem definida é necessário provar a existência de $E$ que satisfaça as hipóteses e que a definição independe da escolha. Neste sentido, a álgebra de Weil

$$
W:=\wedge\left(g^{*}\right) \otimes S\left(g^{*}\right)
$$

é considerada a $G^{*}$-álgebra acíclica mais simples que satisfaz a condição $(\mathbf{C})$. Considerando $x_{1}, \cdots, x_{n}$ uma base de $g^{*}$, podemos ver seus elementos de conexão como sendo $x_{1} \otimes 1, \cdots, x_{n} \otimes 1$.

A simplicidade e descrição de $W$ possibilita uma algebrização que, não só facilita, como, de certa forma, justifica o uso da cohomologia equivarinte de $G^{*}$-módulos. Em outras palavras, com a estrutura algébrica de um $G^{*}$ módulo junto com a teoria desenvolvida pela álgebra de Weil, garantimos uma descrição mais precisa da cohomologia equivariante. Para simbolizar isto, temos uma descrição dos elementos horizontais de $W$ como o grupo de simetrias de $g^{*}$ ou, simplesmente, polinômios em $\mathbb{C}$ com variáveis em $W_{\text {hor }}$, ou seja,

$$
W_{\text {hor }}=\mathbb{C}\left[\mu^{1}, \cdots, \mu^{n}\right]=S\left(g^{*}\right),
$$

onde $\mu^{i}$ são os chamados elementos de curvatura que são elementos de grau 2 
em $W$ que satisfaz que $\iota_{j} \mu^{i}=0$.

De onde surge a pergunta: o que a descrição acima nos auxilia no cálculo de $(A \otimes W)_{b a s}$ ? Aqui entra o isomorfismo de Mathai-Quillen. Considere primeiro $B$ um $G^{*}$-módulo e $A$ um $W^{*}$-módulo, isto é, um $G^{*}$-módulo em que o mapa

$$
W \otimes A \rightarrow A, w \otimes a \mapsto w a
$$

é um morfismo de $G^{*}$-módulos. Podemos criar um automorfismo de $A \otimes B$ que leva $(A \otimes B)_{h o r}$ em $A_{\text {hor }} \otimes B$, este é o isomorfismo de Mathai-Quillen. Este isomorfismo leva $\mathcal{L}_{\xi}$ em $\mathcal{L}_{\xi}$, porém deixa a derivação $d$ um pouco mais complexa, isto é, $d$ é levado em

$$
\sum_{k} d-\mu^{k} \otimes \iota_{k}+\theta^{k} \otimes \mathcal{L}_{k}
$$

onde $\theta^{k}$ são elementos de conexão e $\mu^{k}$ elementos de curvatura de $W$.

Como $\theta^{k}$ são as formas de conexão, considerando $A=W$, temos que o mapa de Mathai-Quillen garante o isomorfismo

$$
(W \otimes B)_{b a s} \rightarrow\left(S\left(g^{*}\right) \otimes B\right)^{G} .
$$

Desta forma, damos origem ao modelo de Cartan para a cohomologia equivariante de $B$ que considera o espaço

$$
C_{G}(B):=\left(S\left(g^{*}\right) \otimes B\right)^{G}
$$

e o operador diferencial

$$
d_{G}: C_{G}(B) \rightarrow C_{G}(B), \quad d_{G}=1 \otimes d_{B}-\mu^{a} \otimes \iota_{a} .
$$

Portanto, o isomorfismo de Mathai-Quillen garante que

$$
\left.H^{*}\left((W \otimes B)_{b a s}\right), d\right)=H^{*}\left(C_{G}(B), d_{G}\right)
$$

Mas o que realmente ganhamos com está nova representação? Para começar, é importante para o nosso resultado final que identifiquemos

$$
H_{G}(\Omega(M))=H^{*}(M / G)
$$

Ora, mas com a nova identificação podemos provar que se $A$ é um $W^{*}$-módulo 
acíclico e $E$ uma $W^{*}$-álgebra acíclica então

$$
H^{*}\left((A \otimes E)_{b a s}\right)=H^{*}\left(A_{b a s}\right) .
$$

Assim, como as formas básicas em $\Omega(M)$ são difeomorfas a $\Omega(M / G)$, temos a identificação que queremos. Além disso, ainda graças a este teorema, é imediato que se $E$ e $F$ são $G^{*}$-álgebras acíclicas que satisfazem a condição $(\mathbf{C})$, então

$$
H_{b a s}^{*}(A \otimes E)=H_{b a s}^{*}(A \otimes F),
$$

mostrando, assim, que a definição da cohomologia equivariante de $G^{*}$ módulo/álgebra está bem definida.

Todavia, o benefício de usar o modelo de Cartan não acaba por aí. Podemos identificar o modelo de Cartan como um bicomplexo e, assim, caso $G$ seja conexo, podemos identificar o primeiro nível da sequência espectral do bicomplexo

$$
C^{p, q}\left(S^{p}\left(g^{*}\right) \otimes A^{q-p}\right)^{G}
$$

como

$$
S^{p}\left(g^{*}\right)^{G} \otimes H^{q-p}(A)
$$

Isto nos permite provar muitas coisas, como o fato de que se um morfismo de grau 0 ,

$$
\rho: A \rightarrow B
$$

de $G^{*}$-módulos induz um isomorfismo na cohomologia standard, ele também induz um isomorfismo na cohomologia equivariante. Mas ainda, graças à teoria de sequências espectrais de complexo duplo, sabemos que a cohomologia do limite da sequência espectral é uma versão graduada da cohomologia equivariante, desta forma, se temos um isomorfismo no primeiro nível de sequências espectrais, temos um isomorfismo entre as cohomologias equivariantes. Assim, considerando $G$ um grupo de Lie compacto e conexo, dado $K$ um subgrupo de Lie de $G$ e $A$ um $G^{*}$-módulo, se provarmos que

$$
S\left(g^{*}\right)^{G} \rightarrow S\left(k^{*}\right)^{K}
$$

é um isomorfismo e, portanto,

$$
S\left(g^{*}\right)^{G} \otimes H(A) \rightarrow S\left(k^{*}\right)^{K} \otimes H(A)
$$


é um isomorfismo, temos que

$$
H_{G}(A) \rightarrow H_{K}(A)
$$

é um isomorfismo.

Então, considerando $T$ o toro maximal de $G$ e $\mathcal{W}$ o grupo de Weil, graças ao teorema de Chevalley que diz que

$$
S\left(g^{*}\right) \rightarrow S\left(t^{*}\right)^{\mathcal{W}} \text { é um isomorfismo, }
$$

temos que

$$
S\left(k^{*}\right)^{K}=S\left(t^{*}\right)^{\mathcal{W}}=S\left(g^{*}\right)^{G},
$$

onde $K$ é o subgrupo de $G$ normalizador de $T$. Assim, é possivel provar que

$$
H_{G}(A) \cong H_{T}(A)^{\mathcal{W}} \quad \text { e } \quad H_{G}(A) \otimes_{S\left(t^{*}\right)^{\mathcal{W}}} S\left(t^{*}\right) \cong H_{T}(A)
$$

Por fim, usando o isomorfismo acima, o isomorfismo de $H^{*}(M / G)$ e $H^{*}(M / T)$ induzida pela submersão

$$
\pi: M / T \rightarrow M / G
$$

que é, basicamente, o mapa do fibrado associado e, ainda, usando os mapas de Chern-Weil (ou simplesmente, homomorfismo característico)

$$
\kappa_{G}: S\left(g^{*}\right)^{G} \rightarrow H^{*}(M / G) \quad \text { e } \quad \kappa_{T}: S\left(t^{*}\right)^{\mathcal{W}} \rightarrow H^{*}(M / T)
$$

prova-se que:

$$
H^{*}(M / T)=H^{*}(M / G)\left[\sigma^{1}, \cdots, \sigma^{r}\right] / I
$$

onde, para $x^{1}, \cdots, x^{r}$ uma base de $t^{*}$ e $p_{i}\left(x^{1}, \cdots x^{r}\right) i=1, \cdots, k$ geradores de $S\left(t^{*}\right)^{\mathcal{W}}$, temos que $\sigma^{j}=\kappa_{T}\left(x^{j}\right)$ e $I$ é o ideal gerado por $\pi^{*}\left(c_{i}\right)-p_{i}\left(\sigma^{1}, \cdots, \sigma^{r}\right)$, $\operatorname{com} c_{i}=\kappa_{G}\left(p_{1}\right)$.

Esta dissertação tem como objetivo fazer a construção acima detalhada, exibindo as definições e construções minuciosamente até chegarmos na fórmula para a cohomologia de fibrados flag homogêneos. Como o leitor poderá ver, a maior parte do trabalho é alto contido, estando as construções feitas se não no texto corrido, no apêndice. Apenas alguns resultados, como o teorema de Chevalley, serão apenas mencionados e não provados. 
No capítulo 2 são apresentadas definições e algumas construções preliminares no sentido de serem estruturas básicas para o trabalho. No capítulo 3 , é feita uma breve motivação para o trabalho e é apresentada a cohomologia equivariante topológica. No capítulo 4, são provadas as equações de Weil. No capítulo 5, são definidos $G^{*}$-módulos/álgebras, a cohomologia equivariante referente e a condição (C). No capítulo 6, é introduzida a álgebra de Weil e algumas de suas estruturas, enquanto que, no capítulo 7, é apresentado o isomorfismo de Mathai-Quillen, o modelo de Cartan e é feito com detalhes o homomorfismo característico. Por fim, no capítulo 8, usando a teoria de sequência espectral de complexo duplo que está no apêndice, provamos o resultado principal do trabalho, a fórmula para a cohomlogia do fibrado flag associado. 


\section{Preliminares}

\section{1}

\section{Cohomologia de de Rham}

Sejam $M$ uma variedade diferenciável e $T^{*} M:=\cup_{p \in M} T_{p}^{*} M$ seu fibrado cotangente, isto é, a união dos espaços cotangentes a cada ponto $p \in M$. Podemos entender os espaços vetorias $\Omega^{1}(M)$ e $\Omega^{k}(M)$ da seguinte forma:

$$
\begin{gathered}
\Omega^{1}(M):=\left\{\alpha: \mathfrak{X}(M) \rightarrow C^{\infty}(M) \mid \alpha \in C^{\infty}(M)-\text { linear }\right\}, \\
\Omega^{k}(M):=\{\beta: \overbrace{\mathfrak{X}(M) \times \cdots \times \mathfrak{X}(M)}^{(k)} \rightarrow C^{\infty}(M) \mid \begin{array}{c}
\beta \in C^{\infty}(M)-\text { linear } \\
\text { e alternado }
\end{array}\}
\end{gathered}
$$

onde $\mathfrak{X}(M)$ é o conjunto dos campos vetoriais $C^{\infty}$ em $M$. Diremos, neste caso, que $\alpha$ é uma 1 -forma e $\beta$ uma $k$-forma. Esta definição nos garante uma estrutura de álgebra graduada onde o produto é comutativo no sentido graduado, isto é, $\left[\gamma_{1}, \gamma_{2}\right]=\gamma_{1} \cdot \gamma_{2}-(-1)^{i j} \gamma_{2} \cdot \gamma_{1}=0$ se $\gamma_{1} \in \Omega^{i}(M)$ e $\gamma_{2} \in \Omega^{j}(M)$. Em geral, nos refirimos ao produto como produto wedge e escrevemos $\wedge=$. Entretando, podemos ver de outra forma a construção de toda esta estrutura que, de fato, é equivalente. Começamos então por definir uma 1-forma:

Diremos que $\omega$ é uma 1 -forma em $M$ se $\omega: M \rightarrow T^{*} M$ é uma seção suave do fibrado cotangente $\pi: T^{*} M \rightarrow M$. Dada uma carta local $\left(V, x^{1}, \cdots, x^{n}\right)$ em $M$, para todo $p \in V$, podemos escrever a 1 -forma $\omega$ em $p$ como

$$
\omega_{p}=\left.\sum_{i=1}^{n} a_{i}(p) d x^{i}\right|_{p}
$$

onde $\left\{d x^{1}, \cdots, d x^{n}\right\}$ é a base dual de $\left\{\frac{\partial}{\partial x^{1}}, \cdots, \frac{\partial}{\partial x^{n}}\right\}$ em cada ponto $p \in V$.

O conjunto das 1 -forma nos dá uma estrutura de espaço vetorial denotada por $\Omega^{1}(M)\left(=\Gamma\left(\wedge^{1} T^{*} M\right)\right)$.

Dado $\alpha, \beta$ 1-formas em $M$, definindo o produto wedge $\alpha \wedge \beta$ de forma que

$$
(\alpha \wedge \beta)\left(v_{1}, v_{2}\right)=\alpha\left(v_{1}\right) \beta\left(v_{2}\right)-\alpha\left(v_{2}\right) \beta\left(v_{1}\right) .
$$


Isto nos dá uma estrutura de produto, além da construção de uma 2-forma de $M$. Indutivamente, $\alpha, \beta, i, j$-formas, respectivamente, temos que o produto wedge das duas é dado de tal forma que

$$
(\alpha \wedge \beta)\left(v_{1}, \cdots, v_{i+j}\right)=\sum(\operatorname{sgn} \sigma) \alpha\left(v_{\sigma(1))}, \cdots, v_{\sigma(i)}\right) \beta\left(v_{\sigma(i+1)}, \cdots, v_{\sigma(i+j)}\right)
$$

Produzindo uma $(i+j)$-forma e nos permitindo definir o espaço vetorial $\Omega^{i+j}(M)=\Gamma\left(\wedge^{i+j} T^{*} M\right)$ dado pelas $(i+j)$-formas.

Assim, temos a estrutura de álgebra graduada dada por

$$
\Omega^{*}(M)=\bigoplus_{k=0}^{n} \Omega^{k}(M)
$$

onde $\Omega^{0}(M)=C^{\infty}(M)$.

Se $\alpha \in \Omega^{i}(M)$ dizemos que $\alpha$ tem grau $i$, ou, simplesmente escrevemos $\operatorname{deg} \alpha=i$. Além disso, podemos escrever $\Omega(M)$ em vez de $\Omega^{*}(M)$ quando não estivermos preocupados com o grau das formas e, o nomeamos de complexo de de Rham de $M$.

O operador diferencial

$$
d: \Omega^{q}(M) \rightarrow \Omega^{q+1}(M)
$$

é uma derivação de grau 1 e, portanto, uma derivação de grau ímpar. Ele, localmente, satisfaz o seguinte:

- se $f \in \Omega^{0} M$ então $d f=\sum \frac{\partial f}{\partial x^{i}} d x^{i}$

- se $\omega=\sum f^{I} d x^{I}=\sum d f^{I} d x^{I}$, onde $I$ representa um superíndice.

Uma propriedade importante do operador $d$ é que ele é uma antiderivação. Provemos isto no lema:

\section{Lema 2.1.1}

$$
d(\alpha \wedge \beta)=(d \alpha) \wedge \beta+(-1)^{\operatorname{deg} \alpha} \alpha \wedge d \beta
$$

Prova: Basta verificar localmente. Sejam

$$
\alpha=f_{I} d x_{I}, \quad \beta=g_{J} d x_{J}
$$

Assim,

$$
d(\alpha \wedge \beta)=d\left(f_{I} g_{J}\right) d x_{I} \wedge d x_{J}=\left(d f_{I}\right) g_{J} d x_{I} \wedge d x_{j}+f_{I} d g_{J} d x_{I} \wedge d x_{J}=
$$




$$
=(d \alpha) \wedge \beta+(-1)^{\operatorname{deg} \alpha} \alpha \wedge d \beta
$$

O produto no complexo de de Rham é sempre o wedge, então assim como as notações de produto em geral, ocultaremos o wedge. Portanto, em vez de escrever $\alpha \wedge \beta$, escreveremos apenas $\alpha \beta$.

Provemos agora o seguinte:

\section{Teorema 2.1.2}

$$
d^{2}=0
$$

Prova: Basta verificar localmente. Considere $g \in \Omega^{0}(M)$.

$$
d^{2} g=d\left(\sum_{k} \frac{\partial g}{\partial x_{k}} d x_{k}\right)=\sum_{k, l} \frac{\partial^{2} g}{\partial x_{l} \partial x_{k}} d x_{j} d x_{i} .
$$

Assim, pela antissimetria de $d$ e simetria de $\frac{\partial^{2} g}{\partial x_{l} \partial x_{k}}, d^{2} g=0$, logo $d^{2} \omega=d^{2}\left(g_{I} d x_{I}\right)=0$ e $d^{2}=0$.

Podemos então definir:

Definição 2.1.3 A cohomologia de de Rham de grau $k$ de $M$ é dado por

$$
H_{D R}^{k}(M)=\{k \text {-formas fechadas }\} /\{k \text {-formas exatas }\}
$$

onde $\omega \in \Omega^{k}(M)$ é uma forma fechada se $d \omega=0$ e, além disso, é dita exata se $\omega=d \beta$ com $\beta \in \Omega^{k-1}(M)$. Perceba que nesta cohomologia, o operador fronteira é o operador $d$.

Se não houver dúvidas quanto a cohomologia usada, ou, nessecidade de expressar qual cohomologia está sendo empregada, para simplificar a notação, iremos suprimir o subíndice e escrever simplesmente $H^{k}(M)$ ou $H^{*}(M)$.

Considerando $M, N$ variedades diferenciáveis, dado um mapa $f: M \rightarrow N$ $C^{\infty}$, seu pullback $f^{*}: \Omega^{*}(N) \rightarrow \Omega^{*}(M)$, comuta com o operador $d$, como podemos ver em $[\mathrm{Tu}]$. Por isso, mapas na categoria de variedade diferenciáveis induzem mapas no sentido oposto em suas cohomologias. Em outras palavras, o funtor $\Omega^{*}$ é contravariante.

Vejamos um resultado importante e interessante sobre a Cohomologia de de Rham. 
Como em geral em cohomologias, a Cohomologia de de Rham é um invariante homotópico. Para ser mais preciso, por [BT], considere o seguinte teorema:

\section{Teorema 2.1.4 (Axioma de Homotopia para Cohomologia de de Rham)}

Mapas homotópicos induzem o mesmo mapa na cohomologia.

O uso do termo axioma já nos indica que o resultado é uma propriedade esperada para a cohomologia. Na verdade, ele é um dos cinco axiomas de Eilenberg-Steenrod, que são um conjunto de propriedades esperadas em uma cohomologia/homologia. Os axiomas são: Homotopia, Excisão, Dimensão, Aditividade e Exatidão. Vale observar que o axioma de aditividade em nosso caso será visto na fórmula de Künneth (teorema 3.0.1).

Um resultado imediato do axioma de homotopia do teorema anterior é que duas variedades com o mesmo tipo de homotopia tem a mesma cohomologia e, portanto, se $Z$ é um retrato de deformação de $M$, então $Z$ e $M$ têm a mesma cohomologia. Em particular, se $M$ é contrátil, a cohomologia de $M$ é trivial para grau maior que 0 e $\mathbb{R}$ para grau 0 , ou simplesmente, como também iremos dizer, $M$ é acíclico. Repare que também fazemos uso de que a cohomologia de de Rham de um ponto é trivial para grau maior que 0 e $\mathbb{R}$ para grau 0. Este é o axioma de dimensão.

Podemos concluir também o famoso Lema de Poincaré. Pelas ideias acima, temos que $H^{*}\left(\mathbb{R}^{n}\right)=H^{*}(p)$ onde $p$ é um ponto qualquer em $\mathbb{R}^{n}$, ou de forma mais geral, $H^{*}\left(M \times \mathbb{R}^{n}\right)=H^{*}(M)$. O Lema de Poincaré está de acordo com a fórmula de Künneth (teorema 3.0.1) já que

$$
H^{*}\left(M \times \mathbb{R}^{n}\right) \cong H^{*}(M) \otimes H^{*}\left(\mathbb{R}^{n}\right)
$$

também verifica o resultado.

\section{2}

\section{Grupos de Lie e Fibrado Principal}

Antes de começarmos a falar sobre fibrados, façamos algumas considerações sobre grupos de Lie. Em geral, se $G$ é um grupo de Lie, usaremos a notação $g$ para representar sua álgebra de Lie.

Definição 2.2.1 Um grupo $G$ é dito grupo de Lie se tem uma estrutura de variedade diferenciável na qual as operações

$$
p: G \times G \rightarrow G, \quad p(a, b)=a b
$$


$e$

$$
i: G \rightarrow G, \quad i(a)=a^{-1}
$$

$s \tilde{a} o C^{\infty}$.

Nos grupos de Lie, temos ações naturais de $G$ sobre si mesmo, dadas pelas translações à direita e à esquerda, isto é, dado $a \in G, L_{a}: G \rightarrow G$ com $L_{a}(b)=a b$ e $R_{a}: G \rightarrow G$ com $R_{a}(b)=b a$, ambas sendo aplicações $C^{\infty}$.

Os fibrados tangente e cotangente de $G$ podem ser trivializados através das translações, pois, dado $a \in G, L_{a}$ é claramente um difeomorfismo, ou seja, $d\left(L_{a}\right)$ é um isormofismo entre $T_{1} G$ e $T_{a} G$. Desta forma $G \times T_{1} G \rightarrow T G$ é um difeomorfismo. De forma análoga, para o fibrado cotangente, a transposta $d\left(L_{a^{-1}}\right)_{1}^{*}: T_{1}^{*} G \rightarrow T_{a}^{*} G$ é um isomorfismo e define o difeomorfismo $G \times T_{1}^{*} G \rightarrow T^{*} G$. O mesmo vale para a translação à direita.

Um campo vetorial $\xi$ em $G$ é dito invariante à esquerda se para todo $a \in G,\left(L_{a}\right)_{*} \xi=\xi$, isto é, $d\left(L_{a}\right)_{b}(\xi(b))=\xi(a b)$ para todo $b \in G$. A definição é análoga para campo invariante à direita. Repare que os campos invariantes são determinados pelos seus valores no elemento neutro $e \in G$, pois $\xi(a)=d\left(L_{a}\right)_{e}(\xi(e))$. Ou seja, cada elemento em $T_{e} G$ determina um único campo invariante à esquerda (ou à direita). Denotemos $i n v^{R}$ o conjunto dos campos invariantes à direita. Como $\left(R_{a}\right)_{*}$ é linear, o conjunto $i n v^{R}$ é um espaço vetorial. Mais do que isso, é claramente isomorfo a $T_{e} G$.

Definição 2.2.2 Uma álgebra g é dita uma álgebra de Lie se sua operação de produto, denotada $[\cdot, \cdot]$ (ou simplesmente o colchete de Lie), satisfaz as seguintes propriedades:

- Antissimetria. $[\xi, \zeta]=-[\zeta, \xi], \xi, \zeta \in g$

- Identidade de Jacob. $[\xi,[\zeta, \eta]]=[[\xi, \zeta], \eta]+[\zeta,[\xi, \eta]], \xi, \zeta, \eta \in g$.

Em [Mar] temos o seguinte:

Lema 2.2.3 Sejam $\xi$ e $\zeta$ campos invariantes à direita num grupo de Lie G. Então o colchete de Lie $[\xi, \zeta]$ é invariante à direita. O mesmo vale para campos invariantes à esquerda.

Definição 2.2.4 Definimos então a álgebra de Lie de $G, g$, como os campos invariantes à direita, inv ${ }^{R}$, com a operação de colchete de Lie como produto. 
As definições de $G$ e $g$ nos permite uma representação natural de $G$ em $g$, dado pela representação adjunta. Podemos construir, para todo $a \in G$, a função $c_{a}:=L_{a} \circ R_{a^{-1}}$, dessa forma, para $b \in G, c_{a}(b)=a b a^{-1}$. Definimos então a representação adjunta $A d: G \rightarrow G L(g)$ de $G$ em sua álgebra de Lie $g$ como:

$$
A d(a)=d\left(c_{a}\right)_{e}=d\left(L_{a} \circ R_{a^{-1}}\right)_{e}=d\left(R_{a^{-1}} \circ L_{a}\right)_{e}=\left(d R_{a^{-1}}\right)_{a} \circ\left(d L_{a}\right)_{e}
$$

Estamos mais preparados agora para estudar as estruturas de fibrados que queremos.

Uma submersão sobrejetiva $\pi: M \rightarrow X$ é um fibrado, (localmente trivial), com fibra $F$ se, para todo $p \in X$, existe vizinhança aberta $U \subset X$ de $p$ e um difeomorfismo $\Phi_{U}$ tal que

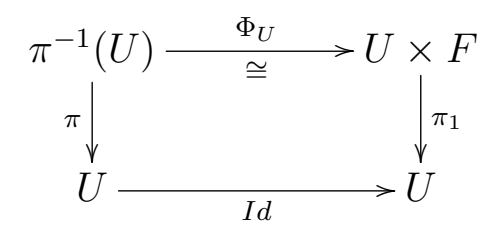

é um diagrama comutativo, onde $\pi_{1}$ é a projeção na primeira coordenada. Dessa forma, dada uma cobertura $\left\{U_{\alpha}\right\}$ de $X$ e difeomorfismos que preservam a fibra $\Phi_{\alpha}: \pi^{-1}\left(U_{\alpha}\right) \rightarrow U_{\alpha} \times F$, temos que para todo $p \in X$, se $p \in U_{\alpha} \cap U_{\beta}$ $\left(U_{\alpha}, U_{\beta} \in\left\{U_{\alpha}\right\}\right)$, então

$$
\phi_{\alpha \beta}(p)=:\left.\Phi_{\alpha} \Phi_{\beta}^{-1}\right|_{\{p\} \times F}
$$

pertence ao subgrupo dos difeomorfismos de $F$, ou simplesmente $\operatorname{Diff}(F)$.

Seja $H<\operatorname{Diff}(F)$ um subgrupo. Diremos que $\pi: M \rightarrow X$ tem grupo estrutural $H$ se existe cobertura $\left\{U_{\alpha}\right\}$ de $X$ e difeomorfismos $\left\{\Phi_{\alpha}\right\}$ tal que $\phi_{\alpha, \beta}(p) \in H$ para todo $p \in U_{\alpha} \cap U_{\beta} \operatorname{com} U_{\alpha}, U_{\beta} \in\left\{U_{\alpha}\right\}$.

Um fibrado principal pode ter uma definição mais geral que independe de uma estrutura diferencial, como em [Hus] que considera apenas grupos topológicos. No nosso caso, consideraremos grupos de Lie, isto é,

Definição 2.2.5 Seja G um grupo de Lie. Um fibrado é chamado de fibrado principal se sua fibra é $G$ e seu grupo estrutural é $G^{R}$, onde $G^{R}:=\left\{R_{a}: G \rightarrow\right.$ $G \mid a \in G\}$, ou seja, o grupo das translações à direita.

Repare que temos um isomorfismo canônico entre $G$ e $G^{R}$, portanto, podemos escrever, sem perda de generalidade, o subgrupo estrutural como 
sendo o próprio $G$.

Teorema 2.2.6 Se $\pi: M \rightarrow X$ é um fibrado principal com fibra $G$, então $G$ age livremente em $M$, isto é, se $\rho$ define a ação de $G$ em $M$, se a $\in G$ e $\rho(a) p=p$ para algum $p \in M$ então $a=e$ (identidade do grupo $G$ ).

Para simplificar a notação, escreveremos simplesmente ap em vez de $\rho(a) p$.

Prova: Considere uma trivialização local $\Phi$, temos então $\pi^{-1}(U) \cong U \times G$. A ação de $G$ em $M$ é dada por ação canônica de $G$ em $X \times G$. Assim, sejam $p \in M \Phi(p)=(v, b) \in X \times G$ e $a \in G$. Temos que $\Phi(a p)=\left(v, R_{a} b\right)$ e $\left(v, R_{a} b\right)=(v, b)$ se e somente se $a=e$, onde $e$ é o elemento neutro de $G$. Assim, o resultado segue.

Definição 2.2.7 Um subgrupo de $G, H<G$, é um subgrupo de Lie se $H$ é uma subvariedade imersa de $G$ tal que as operações p e i restritas a $H$ são $C^{\infty}$ em relação a estrutura de $H$ herdada de $G$.

Em [Mar] podemos ver que se $H$ é um subgrupo fechado de $G$, então $H$ é um subgrupo de Lie, ao mesmo tempo que se $H<G$ é um subgrupo de Lie mergulhado, então $H$ é fechado.

Outro resultado importante que temos é o seguinte:

Teorema 2.2.8 Suponha que $H$ seja um grupo de Lie que age livremente em $M$. Se existe uma estrutura de variedade em $M / H$ tal que $\pi: M \rightarrow M / H$ é uma submersão, então ela é unica a menos de difeomorfismo.

Prova: A prova deste teorema é razoávelmente simples. Considere o seguinte diagrama

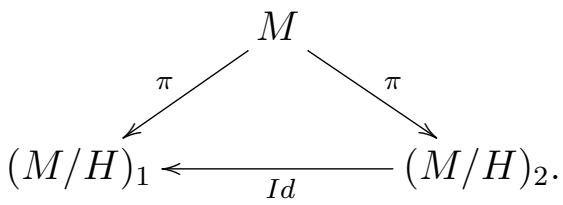

onde $(M / H)_{1}$ e $(M / H)_{2}$ representam apenas atlas diferentes de $M / H$. Para garantir o teorema basta verificar que o mapa identidade $I d$ é $C^{\infty}$. Ora, mas isso é imediato, basta lembrar que, localmente, Id é a composição de seções locais suaves com $\pi$, logo o resultado segue. 
Vale observar que o teorema é mais geral, só usamos na prova que $M / H$ admite estrutura suave tal que $\pi$ é uma submersão. Desta forma, se considerarmos que $M / \sim$ admite uma estrutura tal que $\pi$ é uma submersão, o resultado é válido para qualquer que seja a relação de equivalência $\sim$.

Agora, talvez mais forte que o resultado anterior, temos o teorema:

Teorema 2.2.9 Se Gé um grupo de Lie e $H<G$ é um subgrupo fechado, então existe uma estrutura de variedade em $G / H$ compativel com a topologia quociente na qual a projeção canônica $\pi: G \rightarrow G / H$ é uma submersão.

A demonstração deste teorema é extremamente técnica. A dificuldade maior está em provar que $G / H$ é localmente Euclidiano e que conseguimos obter uma cobertura via sistema de coordenadas para $G / H$. Então podemos dividir a demonstração em duas partes, a primeira é mostrar que $G / H$ tem base enumerável e tem a propriedade de ser Hausdorff. O que, com um pouco de trabalho, podemos mostrar que é consequência do mapa $\pi: G \rightarrow G / H$ ser aberto.

A segunda etapa da demonstração é mostrar que $G / H$ é localmente Euclidiano e que é possivel cobrir $G / H$ por um sistema de coordenadas. A prova disto não é nada simples e usa inicialmente o teorema de Frobenius para construir abertos e mapas que nos permitirá verificar que $G / H$ é uma variedade e $\pi: G \rightarrow G / H$ uma submersão.

Como falamos, a prova é um pouco técnica e longa, então, para não atrapalhar o fluxo deste trabalho, convido o leitor a conferir a prova do resultado no apêndice $\mathrm{A}$.

Perceba que de forma análoga ao teorema 2.2.8, a estrutura diferenciável de $G / H$ que satisfaz o teorema é única a menos de difeomorfismo.

Já vimos que se $\pi: M \rightarrow X$ é um fibrado principal com fibra $G$, então $G$ age livremente em $M$. Agora, o que procuramos saber é o contrário, para ser mais preciso, sobre que condições $G$ agir livremente sobre $M$ nos gera um fibrado principal. Então vejamos o seguinte teorema:

Teorema 2.2.10 Seja G um Grupo de Lie compacto que age livremente em uma variedade $M$, então $M / G$ é uma variedade e $\pi: M \rightarrow M / G$ é um fibrado principal com fibra $G$. 
Uma outra forma de escrever $\pi: M \rightarrow X$ um fibrado principal com fibra e grupo estrutural $G$ é simplesmente $\pi: M \rightarrow X$ um $G$-fibrado principal. Ou seja, no caso do teorema anterior, $\pi: M \rightarrow M / G$ é um $G$-fibrado principal.

Daremos uma ideia da demonstração do teorema 2.2.10. Primeiro tentemos ver $G$ mergulhado em $M$ através de sua órbita em um ponto, isto é, seja $\rho$ a ação de $G$ em $M$, isto nos gera um mapa $\alpha$ de $G \times M$ em $M$ tal que $\alpha(a, p)=\rho(a) p$. Este mapa é suave pois a ação de $\rho$ é suave. Assim, fixando $p \in M$, temos que o mapa $\alpha_{p}: G \rightarrow M$ tal que $\alpha_{p}(a)=\rho(a) p$, também é suave. Afirmamos que este é um mergulho de $G$ em $M$.

De fato, repare primeiro que $\alpha_{p}$ é injetivo, consequência imediata da ação de $G$ ser livre. Outra consequência da ação ser livre é a imersão, mas esta não é tão direta. Seja $\xi \in g \cong T_{e} G$, onde $g$ é a álgebra de Lie de $G$, tal que

$$
d(\alpha)_{e}(\xi)=\left.0 \Rightarrow \frac{d}{d t} \alpha(\exp t \xi)(e)\right|_{t=0}=0
$$

$\left.\operatorname{mas} \frac{d}{d t} \alpha(\exp t \xi)(e)\right|_{t=0}=0 \Rightarrow \xi=0$ pois a ação é livre. Caso contrário, existiria uma vizinhança de 0 tal que $\alpha(\exp t \xi)$ é constante nessa vizinhança.

Assim, como a translação é um difeomorfimos, temos o resultado, isto é, como claramente $\alpha_{\rho(a) p}=\alpha_{p} \circ R_{\rho(a)}$, dado $\chi \in T_{a} G$, temos que

$$
d(\alpha)_{a}(\chi)=d\left(\alpha_{p} \circ R_{\rho(a)}\right)_{e} \circ\left(d R_{\rho\left(a^{-1}\right)}\right)_{\rho(a)}(\chi)=0 .
$$

E como, pelo feito anteriormente, $d\left(\alpha_{p} \circ R_{\rho(a)}\right)_{e}$ é injetivo, $d(\alpha)_{a}(\chi)$ é injetivo.

Vale comentar que essa construção garante que se a ação de $G$ em $M$ é livre, a ação de $G$ em $M$ é localmente livre (definição 5.3.1).

E por fim, como $G$ é compacto e $M$ é hausdorff, $\alpha_{p}$ é um homeomorfismo sobre sua imagem e portanto, um mergulho de $G$ em $M$. Chamemos de $G \cdot p$ a imagem de $G$ por $\alpha_{p}$.

Temos então que $G \cdot p$ é uma subvariedade de $M$, então centrada em $p$, existe uma vizinhança no qual há um sistema de coordenadas $x_{1}, \ldots, x_{m}$ tal que $x_{1}=\ldots=x_{k}=0$ quando restrito a $G \cdot p$, sendo $m$ a dimensão de $M$ e $k$ a dimensão de $G$. Considere $t$ uma seção transversal de $G \cdot p$, ou seja, neste sistema de coordenadas $x_{k+1}=\ldots=x_{m}=0$ quando restrito a $t$. Assim, considere um mapa $\beta: G \times t \rightarrow M \operatorname{com} \beta(a, u)=\rho(a) u$, o que nada mais é 
que o mapa avaliação restrito a $t$.

Note que, como $G$ age livremente em $M$ e em particular em $p$, por [DK] existe uma vizinhança de $p$ em $M$ que é $G$-invariante, isto é, existe uma vizinhança $U$ da órbira de $p, G \cdot p$, que é $G$-invariante. Diminuindo a vizinhança $U$ mencionada acima se necessário, também por [DK], existe um difeomorfismo dado por $\beta$ entre a interseção de uma vizinhança de $p$ com $t$, e $U$, isto é, no sistema de coordenadas locais centrada em $p$, existe $B(0, \delta) \subset \mathbb{R}^{m}$ tal que $t[\delta]=t \cap B(0, \delta)$ e $\beta: G \times t[\delta] \rightarrow U \subset M$ é um difeomorfismo. Assim, $G \times t[\delta] \cong U$ e portanto, $\pi(U) \cong U / G \cong t[\delta]$.

Assim, temos que $\pi(U)=: \Upsilon$ é uma vizinhança aberta de $\pi(p)$ em $M / G$ e também, $\Upsilon \cong t[\delta]$. Consideremos a maximilização de $\left\{\Upsilon_{p} \mid p \in M\right\}$ como o atlas de $M / G$. Mostremos que a mudança de cartas é $C^{\infty}$. Para fazer isso, iremos usar fortemente a relação $\Upsilon \cong t[\delta]$. Sejam $\Upsilon_{1}, \Upsilon_{2}$ cartas em $M / G$. Isso nos gera vizinhanças $U_{1}, U_{2}$ em $M$ e seções $t_{1}, t_{2}$ associadas. Assim, por construção, estudar a mudança de cartas $\Upsilon_{1}, \Upsilon_{2}$ é o mesmo que estudar a transição entre a parte de $t_{1}$ que está contido em $U_{2}$ para a parte de $t_{2}$ que está contido em $U_{1}$, ou seja, $t_{1} \cap U_{2} \rightarrow t_{2} \cap U_{1}$.

Ora, mas $t_{1} \cap U_{2}$ é uma imersão injetiva em $G \times\left(t_{1} \cap U_{2}\right)$ através do mapa $z \rightarrow(e, z)$ e, por construção, $G \times\left(t_{1} \cap U_{2}\right) \cong U_{1} \cap U_{2}$. De forma análoga, temos um mapa $C^{\infty}$ de $t_{2} \cap U_{1}$ em $U_{1} \cap U_{2}$. Logo

$$
t_{1} \cap U_{2} \longrightarrow U_{1} \cap U_{2} \stackrel{\cong}{\longrightarrow} G \times\left(t_{2} \cap U_{1}\right) \stackrel{\pi_{0}}{\longrightarrow} t_{2} \cap U_{1}
$$

onde $\pi_{0}$ é a projeção na segunda coordenada, é uma composição de mapas $C^{\infty}$, portanto $C^{\infty}$.

Concluímos então que $M / G$ é localmente Euclidiana e tem estrutura diferenciável, como $M / G$ é Hausdorff e tem base enumerável (a prova disto é análoga ao feito no teorema 2.2.9 no apêndice A), temos que $M / G$ é uma variedade diferenciável.

Temos que mostrar agora que $\pi: M \rightarrow M / G$ é $C^{\infty}$. Ora, mas consideremos novamente $U$ vizinhança $G$-invariante de $G \cdot p$ e $\Upsilon=\pi(U) \cong t[\delta]$. Chamemos de $\psi$ o difeomorfismo entre $\Upsilon$ e $t[\delta]$. Assim, restrito a $U$ temos a igualdade

$$
\pi=\beta \circ(I d \times \psi) \circ \pi_{0}
$$


pelo diagrama comutativo

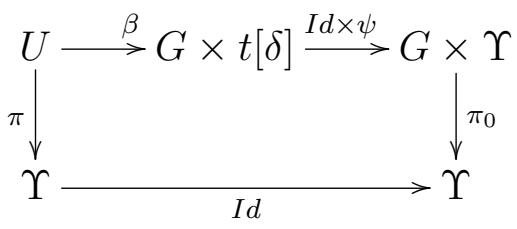

Como a composição é $C^{\infty}, \pi$ é $C^{\infty}$. E finalmente, como as funções de transição também podem ser dadas como composição de mapas suaves como acima, elas são $C^{\infty}$. Logo $\pi: M \rightarrow M / G$ é um $G$-fibrado principal como queríamos.

Vale comentar que na construção acima consideramos a ação de $G$ em $M$ pela esquerda, todavía a construção é análoga para a ação pela direita.

\section{3}

\section{Fibrado Associado e Fibrado Flag Homogêneo}

Agora, considere $\pi: M \rightarrow X$ um $G$-fibrado principal e uma ação de $G$ em uma variedade diferenciável $F$.

Temos então uma ação natural a partir da diagonal de $G \times G$ em $M \times F$, ou, simplesmente uma ação de $G$ em $M \times F$ dado por $a(p, q)=\left(p a, a^{-1} q\right)$ onde $a \in G$ e $(p, q) \in M \times F$. Essa ação é claramente livre, pois a ação de $G$ em $M$ o é, e define uma relação de equivalência em $M \times F$.

Definição 2.3.1 Sejam $\pi: M \rightarrow X$ um fibrado principal com fibra $G$ e que $G$ age em uma variedade $F$ pela esquerda. Definimos o fibrado associado como sendo $[\pi]: P=(M \times F) / G \rightarrow X$, que tem como fibra $F$.

Repare que esta construção está bem definida pois se $(p, q) \sim(s, t)$ em $P$, então $p$ e $s$ estão na mesma fibra em $M, \operatorname{logo}[\pi](p, q)=\pi(p)=\pi(s)=[\pi](s, t)$.

Sejam $\pi: M \rightarrow X$ um $G$-fibrado principal e $G / H$ um espaço homogêneo de $G$. Temos uma ação natural de $G$ em $G / H$ pela multiplicação, então caso $G / H$ tenha uma estrutura de variedade, recaímos nas hipóteses da definição de fibrado associado, nos permitindo definir:

Definição 2.3.2 Um fibrado homogêneo é um fibrado associado no qual a fibra é $G / H$, onde $H$ é um subgrupo de Lie de $G$, isto é, $F=G / H$.

É suficiente que o subgrupo $H$ seja fechado para que $G / H$ seja uma variedade, então poderíamos definir o fibrado homogêneo diretamente supondo que $H$ é fechado, assim como em [Mar]. 
Vale ressaltar que, em geral, iremos trabalhar considerando $G$ um grupo de Lie compacto (e conexo), desta forma, pelo $G$-fibrado principal $\pi: M \rightarrow X$, temos que $M / G$ é uma variedade e podemos identificar de maneira natural, $X$ e $M / G$, isto é,

$$
X \cong M / G
$$

Assim, podemos considerar um $G$-fibrado principal simplesmente como $\pi: M \rightarrow M / G$. Além disso, considerando $G$ compacto, temos que $H$ também é compacto e age em $M$ pela restrição, portanto, $M / H$ também é uma variedade. Mas não uma variedade qualquer, afirmo que ele é um fibrado associado homogêneo, isto é, dado

$$
[\pi]: P=(M \times G / H) / G \rightarrow M / G
$$

o fibrado associado homogêneo, temos que

$$
(M \times G / H) / G \cong M / H
$$

De fato, defina $1_{G / H}$ a origem de $G / H$. Consideremos o diagrama

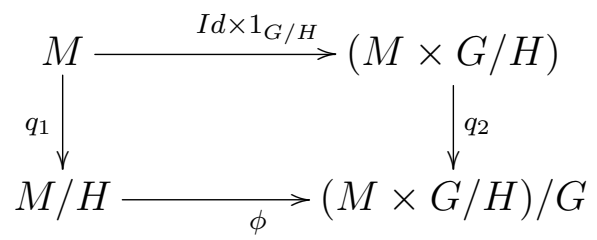

onde $\phi([p])=q_{2} \circ\left(I d \times 1_{G / H}\right)(p)=\left[p \cdot 1_{G / H}\right]$. O mapa está bem definido independente da escolha do elemento na classe de $p$ pois, se $x \in[p]$ então $x=p u$ para algum $u \in H . \operatorname{Logo},\left[x \cdot 1_{G / H}\right]=\left[p u \cdot 1_{G / H}\right]=\left[p \cdot u 1_{G / H}\right]=$ $\left[p \cdot 1_{G / H}\right] \in(M \times G / H) / G$.

Temos que mostrar agora que o mapa é uma bijeção. Mostremos primeiro a sobrejetividade. Considere $[z \cdot x] \in(M \times G / H) / G$. Pela estrutura de grupo, existe $a \in G$ tal que $a^{-1} x=1_{G / H}$. Ou seja,

$$
[z \cdot x]=\left[z \cdot a 1_{G / H}\right]=\left[z a \cdot 1_{G / H}\right]=\left[p \cdot 1_{G / H}\right]
$$

se $p=z a^{-1}$. Logo, $[z \cdot x]$ está na imagem do mapa. Resta provar a injetividade.

Se $\left[p \cdot 1_{G / H}\right]=\left[x \cdot 1_{G / H}\right]$, então existe $a$ tal que $x=p a$ e $a^{-1} \cdot 1_{G / H}=1_{G / H}$. Logo $a \in H$ e $[p]=[x]$, provando assim a injetividade.

Logo o mapa $\phi$ é bijetivo. Além disso, o mapa é feito de forma que 
o diagrama 2.3.1 seja comutativo. Mas ambas as ações são suaves, portanto é possível ver que o mapa $\phi$ é suave. Porém, mais do que isso, afirmo que esse mapa é um difeomorfismo. Em [Mar], o autor faz a passagem de forma semelhante à que construímos acima (no sentido oposto), onde nos garante o difeomorfismo, isto é, $M / H$ é difeomorfo ao fibrado associado homogêneo, ou seja,

$$
M / H \cong(M \times G / H) / G
$$

Assim, podemos simplificar o fibrado associado pelo diagrama

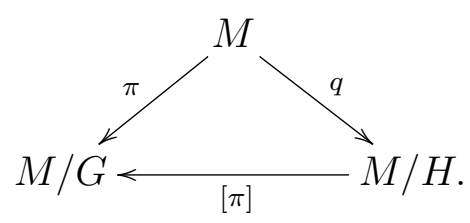

Um exemplo importante de subgrupo de Lie de $G$ fechado é o toro maximal.

Definição 2.3.3 Um toro $T$ é um grupo de Lie compacto, conexo e abeliano. Se o toro $T$ é um subgrupo de Lie de $G$, dizemos que $T$ é um toro em $G$. Em particular, se $T$ não está contido própriamente em nenhum outro toro em $G$, dizemos que $T$ é um toro maximal em $G$.

Vale ressaltar duas observações, caso $G$ seja compacto, $G$ admite toros entre seus subgrupos, incluindo o toro maximal. Mais ainda, caso $G$ seja compacto e conexo, temos que o toro maximal $T$ é um subgrupo de Cartan, isto é, tem como álgebra de Lie uma subálgebra de Cartan, ou seja, se $t$ é a álgebra de Lie de $T$, então $t$ é nilpotente e ele é seu próprio normalizador (se $\xi \in g$, então $[\xi, t] \in t$ se, e somente se, $\xi \in t$ ). De fato, por [Mar], como $G$ é compacto, se $u \subset g$ é uma subálgebra de Cartan, então $u$ é abeliana maximal. Como $t$ é conexo e uma subálgebra de Cartan de $g$ é maximal, $t$ está contido inteiramente em uma subálgebra de Cartan, a denotemos por $u$. Portanto $<\exp u>$ é um grupo abeliano e conexo contido em $G$. Mas $G$ é compacto, portanto o fecho de $<\exp u>$ também está em $G$, além de ser conexo e abeliano. Logo, o fecho de $<\exp u>$ é um toro que contém $T$. Como $T$ é maximal, temos a igualdade e portanto $t=u$.

Podemos, agora, definir o seguinte fibrado:

Definição 2.3.4 Seja G um grupo de Lie compacto e conexo. Um fibrado flag homogêneo é um fibrado homogêneo no qual $H=T$, isto é, a fibra do G-fibrado é $G / T$, onde $T$ é o toro maximal de $G$. 
Considerando então o fibrado principal como sendo $\rho: M \rightarrow M / G$ o diagrama

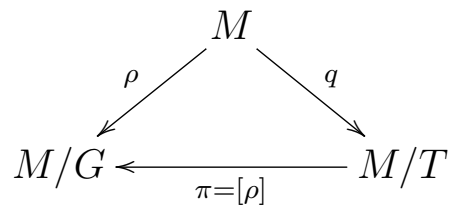

ilustra bem o comportamento do fibrado flag homogêneo, temos que o mapa $\pi$ projeta a órbita de $T$ em $M$ na órbita de $G$ em $M$ como uma submersão. 


\section{3}

\section{Cohomologia de Fibrados}

Neste capítulo vamos dissertar sobre a cohomologia de fibrados. Mas, muito mais do que isto, este capítulo, de certa forma, vai motivar o resultado que buscamos neste trabalho.

Um dos primeiros resultados que podemos observar ao estudar a cohomologia de fibrados vetoriais é a fórmula de Künneth que podemos ver em [BT], o enunciado diz o seguinte:

Teorema 3.0.1 (Fórmula de Künneth) Seja $M$ uma variedade diferenciavel que admite boa cobertura finita e seja $F$ uma variedade diferenciável. Então

$$
H^{n}(M \times F)=\underset{p+q=n}{\oplus} H^{P}(M) \otimes H^{q}(F) .
$$

A hipótese de $M$ ter boa cobertura finita pode ser omitida se supormos que $F$ tenha cohomologia de dimensão finita.

O resultado pode parecer não tão interessante na perspectiva de fibrados por se restringir a fibrados triviais, porém, mais geralmente, esta fórmula pode facilitar o cálculo da cohomologia de variedades que poderia ser mais difícil diretamente.

Exemplo 3.0.2 Considerando que a cohomologia da esfera $\mathbb{S}^{n}$ é igual a $\mathbb{R}$ nos graus 0 e n, e 0 nos outros graus, calculemos a cohomologia de $\mathbb{S}^{1} \times \mathbb{S}^{3}$.

$$
H^{n}\left(\mathbb{S}^{1} \times \mathbb{S}^{3}\right)=\underset{p+q=n}{\bigoplus} H^{P}\left(\mathbb{S}^{3}\right) \otimes H^{q}\left(\mathbb{S}^{1}\right)=
$$

Então,

$$
\begin{aligned}
& \qquad H^{0}\left(\mathbb{S}^{1} \times \mathbb{S}^{3}\right)=H^{0}\left(\mathbb{S}^{1}\right) \otimes H^{0}\left(\mathbb{S}^{3}\right)=\mathbb{R} \otimes \mathbb{R}=\mathbb{R}, \\
& H^{1}\left(\mathbb{S}^{1} \times \mathbb{S}^{3}\right)=H^{1}\left(\mathbb{S}^{1}\right) \otimes H^{0}\left(\mathbb{S}^{3}\right) \oplus H^{0}\left(\mathbb{S}^{1}\right) \otimes H^{1}\left(\mathbb{S}^{3}\right)=(\mathbb{R} \otimes \mathbb{R}) \oplus(\mathbb{R} \otimes 0)=\mathbb{R} . \\
& \text { Como a cohomologia de graus } 1 \text { e } 2 \text { de } \mathbb{S}^{3} \text { é } 0 \text { e } \mathbb{S}^{1} \text { têm cohomologia } 0 \text { para } \\
& \text { graus maiores que } 1 \text {, é fácil ver que }
\end{aligned}
$$

$$
H^{2}\left(\mathbb{S}^{1} \times \mathbb{S}^{3}\right)=0
$$




$$
H^{3}\left(\mathbb{S}^{1} \times \mathbb{S}^{3}\right)=H^{0}\left(\mathbb{S}^{1}\right) \otimes H^{3}\left(\mathbb{S}^{3}\right)=\mathbb{R}
$$

e, por fim,

$$
H^{4}\left(\mathbb{S}^{1} \times \mathbb{S}^{3}\right)=H^{1}\left(\mathbb{S}^{1}\right) \otimes H^{3}\left(\mathbb{S}^{3}\right)=\mathbb{R}
$$

$E$, da mesma forma que $H^{2}\left(\mathbb{S}^{1} \times \mathbb{S}^{3}\right)=0$, é fácil ver que a cohomologia de $\mathbb{S}^{1} \times \mathbb{S}^{3}$ é 0 para graus maiores que 4 . Assim, calculamos a cohomologia de $\mathbb{S}^{1} \times \mathbb{S}^{3}$ facilmente usando a fórmula de Künneth.

Entretanto, o que procuramos, em geral, é escrever, de alguma forma, a cohomologia do fibrado a partir da cohomologia da base e de sua fibra, melhor dizendo, a partir de informações adquiridas da cohomologia da fibra e da variedade base do fibrado. O que, como vamos ver, é possível sobre certas condições.

\section{1 Cohomologia de Fibrados a Partir de Informações Cohomológicas da Base e da Fibra}

De certa forma, o que queremos é que um aspecto puramente geométrico que é a submersão entre um fibrado e sua base traga consequências em cohomologia. Uma das consequências é trivial. Veja:

Consideremos $\pi: M \rightarrow X$ um fibrado. O mapa

$$
\pi^{*}: H^{*}(X) \rightarrow H^{*}(M)
$$

nos define um homomorfismo entre $H^{*}(X)$ e $H^{*}(M)$ e desta forma podemos ver $H^{*}(M)$ como um $H^{*}(X)$-módulo de forma natural, isto é,

$$
H^{*}(X) \times H^{*}(M) \rightarrow H^{*}(M) \quad(x, y) \mapsto \pi^{*}(x) \cdot y
$$

define para $H^{*}(M)$ uma estrutura de $H^{*}(X)$-módulo.

Este é um resultado geral mas não diz muito sobre a cohomologia do fibrado. Um resultado que nos diz bastante sobre a cohomologia de um fibrado a partir de informações cohomológicas da base e da fibra é o poderoso teorema de Leray-Hirsch que também podemos encotrar em [BT], que diz:

Teorema 3.1.1 (Leray-Hirsch) Consideremos $\pi: M \rightarrow X$ um fibrado com fibra $F$ e suponhamos que $X$ admite boa cobertura finita. Se houver classes de cohomologia global $e_{1}, \ldots, e_{r}$ em $M$ que quando restrito a cada fibra geram livremente a cohomologia da fibra, isto é, para todo $b \in X i_{b}^{*} e_{1}, \ldots, i_{b}^{*} e_{r}$ (onde 
$i: \pi^{-1}(b) \rightarrow M$ é a inclusão) é uma base de $H^{*}\left(\pi^{-1}(b)\right)$, então $H^{*}(M)$ é um módulo livre sobre $H^{*}(X)$ com base $\left\{e_{1}, \ldots, e_{r}\right\}$, ou seja,

$$
H^{*}(M) \simeq H^{*}(X) \otimes \mathbb{R}\left\{e_{1}, \ldots, e_{r}\right\} \simeq H^{*}(X) \otimes H^{*}(F)
$$

Exemplo 3.1.2 (Cohomologia de um fibrado esférico) Consideremos

$$
\pi: M \rightarrow X
$$

um fibrado vetorial com fibra de dimensão d e $X$ uma variedade compacta e conexa. O grupo estrutural geralmente é considerado o maior grupo possível, o grupo de difeomorfismos da fibra, porém no nosso caso, como estamos trabalhando com um fibrado vetorial, o grupo que em questão é o de difeomorfismos lineares, ou seja, $G L(d)$. Então, fixando uma métrica Riemanniana, podemos considerar outro fibrado, agora com grupo estrutural $O(d)$. Denotando por $S(M)$, esse é o fibrado de esferas e podemos escrever

$$
S(M) \rightarrow X
$$

Vale ressaltar que podemos ver a fibra como $\mathbb{S}^{d-1}$ já que as órbitas por $O(d)$ são esferas. Dizemos então que um fibrado esférico com fibra $\mathbb{S}^{d-1}$ é orientável se existe uma cobertura aberta $\left\{U_{\alpha}\right\}$ de $X$ e geradores $\left[\sigma_{\alpha}\right]$ de $H^{d-1}\left(\left.S(M)\right|_{U_{\alpha}}\right)$ de forma que $\left[\sigma_{\alpha}\right]=\left[\sigma_{\beta}\right]$ em $H^{d-1}\left(\left.S(M)\right|_{U_{\alpha} \cap U_{\beta}}\right)$. Infelizmente, apesar das sobreposições concordarem, isto não significa que podemos formar uma classe global em cohomologia, pois só significa que, na interseção, elas diferem por uma forma exata.

Entretanto, aqui podemos usar uma outra estrutura, a classe de Euler, que em particular é uma forma global com a dimensão da fibra. Ela mede a existência de uma orientação de cada fibra de $S(M)$ que é localmente constante. Ora, mas

$$
\mathbb{R} \cong H^{d-1}\left(S(M)_{p}\right)
$$

onde $S(M)_{p}$ representa uma fibra de $S(M)$, e, portanto, uma esfera de dimensão $d-1$. Assim, também podemos ver $\mathbb{R}$ a partir da escolha de um gerador de $H^{d-1}\left(\mathbb{S}^{d-1}\right)$. Desta forma, existe $\sigma \in H^{d-1}(S(M))$, pela classe de Euler, tal que $i_{p}^{*} \sigma$ é um gerador de $H^{d-1}\left(\mathbb{S}^{d-1}\right)$, onde $i_{p}: \mathbb{S}^{d-1} \rightarrow S(E)$, caindo assim nas hipóteses do teorema de Leray-Hirsch.

Além disso, $H^{l}\left(S(M)_{p}\right)=0$ se $l \neq 0, d-1$. Desta forma, como podemos ver $1 \in H^{0}(X)$ e $i_{p}^{*} 1$ um gerador de $H^{0}\left(S(M)_{p}\right)$, pelo teorema de Leray-Hirsch, 
temos que

$$
H^{*}(S(M)) \simeq H^{*}(X) \otimes\left(H^{0}\left(\mathbb{S}^{d-1}\right) \oplus H^{d-1}\left(\mathbb{S}^{d-1}\right)\right) \cong H^{*}(X) \otimes \mathbb{R}\{1, \sigma\}
$$

Repare que a equivalência é adequadamente graduada, isto é, $H^{0}(S(M)) \simeq H^{0}(X) \otimes H^{0}\left(\mathbb{S}^{d-1}\right)$ e, para $n \geq d-1, H^{n}(S(M)) \simeq$ $\left(H^{n}(X) \otimes H^{0}\left(\mathbb{S}^{d-1}\right)\right) \oplus\left(H^{n-(d-1)}(X) \otimes H^{d-1}\left(\mathbb{S}^{d-1}\right)\right)$.

Mas detalhes sobre as construções que foram feitas acima podem ser vistas em $[\mathrm{BT}]$.

O teorema de Leray Hirsch é muito poderoso e traz informações valiosas que nos permite calcular a cohomologia do fibrado a partir de informações cohomológicas da base e da fibra. Porém, aqui chamo a atenção que tratamos $H^{*}(M)$ como um módulo livre sobre $H^{*}(X)$ com base $\left\{e_{1}, \ldots, e_{r}\right\}$ e não como anel, ou seja, o isomorfismo no teorema não é um isomorfismo de anéis e $H^{*}(M)$ pode não ser (como em geral não é) equivalente a $H^{*}(X)\left[e_{1}, \cdots, e_{r}\right]$.

É sobre a espectativa de dizer mais sobre a cohomologia de fibrados que vamos trabalhar. Apesar do teorema de Leray Hirsch já ser restrito a alguns tipos de fibrados por causa de suas hipóteses, o resultado que este trabalho tem como objetivo mostrar é ainda mais restrito, ele só funciona com fibrados flag homogêneos. Para chegar neste resultado teremos que iniciar o estudo sobre um tipo específico de cohomologia, a cohomologia equivariante. Ainda para ser mais preciso, a cohomologia equivariante de $G^{*}$-módulos.

Enunciemos então este resultado:

Teorema 3.1.3 (Cohomologia do Fibrado Flag Homogêneo) Seja $M$ uma variedade em que $G$, grupo de Lie compacto e conexo, age livremente. Repare que isto nos gera uma estrutura de fibrado principal dado pela submersão

$$
\pi: M \rightarrow M / G
$$

A cohomologia do fibrado flag homogêneo $(M \times G / T) / G$ é equivalente ao quociente

$$
H^{*}(M / G)\left[\sigma^{1}, \cdots, \sigma^{r}\right] / I
$$

onde I é um ideal gerado, não diretamente, a partir da base do dual da álgebra de Lie de T.

Neste momento é muito complicado explicar precisamente cada termo da equação 3.1.1. O trabalho desta dissertação vai ser, não só provar o teorema 
3.1.3, como também expressar e explicar, cuidadosamente, cada termo da equação 3.1.1. Iremos ver isto no capítulo 8.

\section{2}

\section{Cohomologia Equivariante Topológica}

Estamos usando o termo cohomologia equivariante topológica ou cohomologia equivariante de $G^{*}$-módulo apenas para diferenciar as duas, já que, dependendo do contexto fica clara qual versão estamos usando. Desta forma, podemos por muitas vezes, omitir o termo topológica ou $G^{*}$-módulo.

É claro que neste momento, sem ter nem definido o que seria $G^{*}$-módulo, não falaremos sobre este tipo de cohomologia equivariante, entretanto vale comentar que, sobre certas condições, ambas as cohomologia equivariantes são as mesmas. Este é o teorema equivariante de de Rham e o leitor poderá vê-lo no apêndice B.

Iremos nesta seção fazer uma breve introdução sobre a cohomologia equivariante topológica, todavia, no decorrer desta dissertação iremos focar na cohomologia equivariante de $G^{*}$-módulos. Mas talvez valha aqui ressaltar o porque da escolha deste modelo mais algébrico em relação ao modelo topológico que, como veremos a seguir, pode ser mais direto. O modelo algébrico nos permite generalizar conceitos e trabalhar com estruturas do ponto de vista puramente algébrico, neste sentido ganhamos resultados por efeito de cálculos e teorias ligadas à, por exemplo, teoria de grupos de Lie.

A partir deste momento, tomaremos como base o livro de Victor W. Guillemin e Shlomo Stemberg, "Supersymmetry and equivariant de Rham theory", [GS].

Nesta seção iremos considerar sempre $G$ um grupo de Lie compacto e $M$ uma variedade diferenciável na qual $G$ age. Além disso, iremos usar neste trabalho algumas vezes o termo $G$-equivariante, ele tem a seguinte definição:

Definição 3.2.1 Diz-se que um mapa é G-equivariante se é um homomorfismo da categoria de $G$-conjuntos (conjuntos no qual $G$ age) que estamos trabalhando que comuta com a ação de $G$.

A cohomologia equivariante topológica surge de uma forma muito mais natural que a de $G^{*}$-módulos. Quando temos um $G$-fibrado principal $q: M \rightarrow$ $X$, temos naturalmente que

$$
M / G \cong X
$$


pensemos então de forma oposta.

Caso a ação de $G$ seja livre, pelo teorema 2.2.10, o mapa quociente $\pi: M \rightarrow M / G$ é um fibrado principal, em particular, $M / G$ é uma variedade diferenciável. Desta forma, tem sentido definirmos a cohomologia equivariante como $H_{G}^{*}(M):=H^{*}(M / G)$. Assim, a cohomologia equivariante estuda o espaço das órbitas a partir da ação do grupo de Lie.

Exemplo 3.2.2 Considere o grupo de Lie $O(1)$, o grupo ortogonal de dimensão 1 , ou, simplesmente, o grupo das matrizes $1 \times 1$ que têm determinante igual a 1 ou -1 . Ou seja, $O(1)=\{1,-1\}$. Considere $\mathbb{S}^{n}$ a esfera unitária em $\mathbb{R}^{n+1}$. Temos uma ação natural de $O(1)=\{1,-1\}$ em $\mathbb{S}^{n}$ dado por:

$$
\mathbb{S}^{n} \times O(1) \rightarrow \mathbb{S}^{n}, \text { tal que } x 1=x \text { e } x(-1)=-x \forall x \in \mathbb{S}^{n}
$$

Assim, $\left(\mathbb{S}^{n} / O(1)\right)=\left(\mathbb{S}^{n} /(x \sim-x)\right) \cong \mathbb{R} P^{n}$. Portanto,

$$
H_{O(1)}^{*}\left(\mathbb{S}^{n}\right)=H^{*}\left(\mathbb{R} P^{n}\right)
$$

Apesar da construção feita acima fazer sentido, queremos uma definição que não necessariamente dependa da ação de $G$ em $M$ ser livre. Infelizmente, isso nos gera um problema, não necessariamente $M / G$ é uma variedade diferenciável. Então, como contornar esta situação?

A resposta imediata é substituir a variedade $M$ por uma variedade $M^{*}$ cohomológicamente equivalente a $M$, na qual $G$ tenha uma ação livre. A maneira mais natural de fazer isso é escolher $M^{*}=M \times E$ onde $E$ é um espaço contrátil no qual $G$ age livremente. Assim, pelo teorema 2.1.4, $H^{*}(M)=H^{*}\left(M^{*}\right)$ e pelas nossas considerações no capítulo $2, p: M^{*} \rightarrow M$ é um fibrado associado no qual $G$ age livremente. Desta forma, definimos a cohomologia equivariante de forma geral como:

Definição 3.2.3 A cohomologia $G$-equivariante de $M$, denotada por $H_{G}^{*}(M)$, é definida da seguinte forma:

$$
H_{G}^{*}(M):=H_{G}^{*}\left(M^{*}\right)=H^{*}\left(M^{*} / G\right)=H^{*}((M \times E) / G) .
$$

A definição acima pode ter dois problemas estruturais. Para começar, o que garante que existe um $E$ que satisfaça estas propriedades? Nada garante, a princípio, que tal $E$ exista. Outra questão é a unicidade de $E$. A unicidade neste caso, é no sentido que se escolhermos um $E$ diferente, não teríamos uma 
cohomologia equivariante distinta.

Convido o leitor, caso tenha interesse, olhar o capítulo 1 de [GS], lá se encontram as demonstrações completas destes resultados. Façamos aqui breves comentários sobre a prova.

Primeiro a unicidade, a prova se dá ao seguir dois teoremas. Consideremos o mapa:

$$
j \circ s=: f: M / G \rightarrow E / G
$$

onde $j$ é o mapa do fibrado fibrado associado

$$
j:(M \times E) / G \rightarrow E / G, \quad j:[(x, e)] \mapsto[e]
$$

e $s$ a seção transversal, que sabemos que existe por $E$ ser contrátil,

$$
s: M / G \rightarrow(M \times E) / G
$$

Pode-se provar o seguinte resultado:

Teorema 3.2.4 Qualquer seção transversal $s: M / G \rightarrow(M \times E) / G$ determina um único mapa $G$-equivariante $\epsilon: M \rightarrow E$ que faz o seguinte diagrama comutar

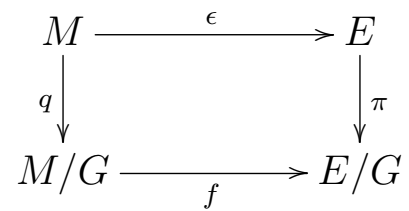

Por outro lado, um mapa G-equivariante $\epsilon: M \rightarrow E$ determina uma seção $s: M / G \rightarrow(M \times E) / G$ e um mapa $f$ que faz com que o diagrama acima comute. E ainda, como quaisquer duas seções são homotópicas, $f, \epsilon$ são únicos a menos de homotopia.

Faremos, posteriormente, um análogo algébrico deste teorema quando tivermos trabalhando com $G^{*}$-módulos. Com o teorema que enunciamos acima é possivel provar:

Teorema 3.2.5 Seja $E_{1}$ e $E_{2}$ variedades contráteis nos quais $G$ age livremente. Então, eles são equivalentes como G-variedade. Isto é, existem Gequivalências

$$
\phi: E_{1} \rightarrow E_{2}, \quad \psi: E_{2} \rightarrow E_{1}
$$


com homotopias G-equivariantes

$$
\psi \circ \phi \simeq I d_{E_{1}}, \quad \phi \circ \psi \simeq I d_{E_{2}}
$$

É imediato do resultado acima que a definição 3.2.3 independe da escolha de $E$, isto é, independe da escolha do espaço contrátil no qual $G$ age livremente.

Quanto a existência de tal $E$ não faremos a prova por inteira. Só faremos algumas considerações que não só são utilizadas na prova da existência, como também são usadas na prova do teorema equivariante de de Rham no apêndice B.

Provar a existência significa provar a existência para qualquer grupo de Lie $G$ compacto. Só que para cada grupo $G$ necessitariamos criar um espaço contrátil diferente para que $G$ possa agir livremente nele, o que é possível, porém, pode ser bem trabalhoso. Então, para faremos a construção, fazemos a seguinte consideração:

Primeiro, todo grupo de Lie compacto tem uma representação linear fiel, ou seja, podemos considerar $G$ como um subgrupo compacto de $G L(m)$, que por sua vez, para $n$ suficientemente grande, pode ser mergulhado em $U(n)=\left\{T \in G L(n, \mathbb{C}) \subset G L(2 n, \mathbb{R}) \mid T^{*} T=I d\right\}$. Ou, simplesmente, podemos dizer como em [Zhe], todo subgrupo de Lie compacto é isomorfo (difeomorfo) a um subgrupo de $U(n)$. Logo, podemos pensar em $G$ contido em $U(n)$ e, é claro, que se $U(n)$ age livremente em um espaço $E, G$ também agirá livremente. Dessa forma, é suficiente achar $E$ que seja conatrátil e admita uma ação livre de $U(n)$.

Consideremos $H=L^{2}[0, \infty)$ o espaço das funções cujos seus quadrados são integráveis em relação a medida de Lebesgue em $[0, \infty) \subset \mathbb{R}$. Esse espaço tem uma métrica natural dada pelo seu produto interno.

Então, definimos o espaço $E$ do seguinte modo:

$$
E:=\left\{f=\left(f_{1}, \ldots, f_{n}\right) \mid f_{i} \in H, \quad\left(f_{i}, f_{j}\right)=\delta_{i j}\right\}
$$

onde $(\cdot, \cdot)$ representa o produto interno em $H$. Essa construção é possível, já que, $H$ é espaço infinito. Mais precisamente, $H$ é o único espaço de Hilbert infinito separável (possui subconjunto denso enumerável ou, equivalentemente, possui um sistema ortonormal completo e enumerável). 
A ação de $U(n)$ em $E$ é dado de forma trivial, dado $A=\left[a_{i j}\right] \in U(n)$ e $f=\left(f_{1}, \ldots f_{n}\right) \in E, A f=A \cdot f^{t}=g=\left(g_{1}, \ldots, g_{n}\right) \in E$ onde $g_{i}=\sum_{j} a_{i j} f_{j}$.

Temos que mostrar que esta ação está bem definida, isto é, se $A \in U(n)$ então $A f \in E$. Para isto, lembremos que $U(n)$ pode ser definido como as matrizes $A$ tais que $A^{*} A=I d$, onde $A^{*}$ é a matriz transposta conjugada de $A$. Podemos pensar de outra forma, graças a definição, se considerarmos $v_{1}, \ldots, v_{n}$ os vetores coluna de $A$, temos que o produto hermitiano de $v_{i}$ e $v_{j}$ é igual a 1 se $i=j$ e 0 caso contrário. Assim, se considerarmos $A f=g=\left(g_{1}, \ldots, g_{n}\right)$, temos que

$$
\begin{gathered}
\left(g_{i}, g_{j}\right)=\left(\sum_{k} a_{i k} f_{k}, \sum_{k} a_{j k} f_{k}\right)=\sum_{k}\left(a_{i k} f_{k}, a_{j k} f_{k}\right)=\sum_{k} a_{i k} \overline{a_{j k}}\left(f_{k}, f_{k}\right)= \\
=\sum_{k} a_{i k} \overline{a_{j k}}=\delta_{i j} .
\end{gathered}
$$

Logo a ação esta bem definida.

É trivial ver que esta ação é livre. O restante da prova seria simplesmente demonstrar que $E$ é contrátil e pode ser vista em [GS].

Vale ressaltar que a construção que fizemos nesta seção não leva em consideração a estrutura diferenciável, ou seja, o próprio $E$ apresentado a pouco não é uma variedade diferenciável. Desta forma a cohomologia empregada não seria a cohomologia de de Rham a princípio. Entretando é possível estender a construção de forma a nos permitir trabalhar com a cohomologia de de Rham. Para começar, é necessário substituir a hipótese de $E$ ser contrátil para uma um pouco mais fraca. Consideraremos $E$ como um espaço tal que, para toda $X$ variedade diferenciável compacta, todo mapa contínuo de $X$ a $E$ é contrátil a um ponto. Esta é, em geral, a definição de um espaço contrátil em uma categoria, no nosso caso, variedades diferenciáveis.

É possível ver que a prova da unicidade ainda é válida. Quanto a existência, podemos considerar $E$ como o espaço final de variedades diferenciáveis, isto é, considerar $E$ como o limite, sobre a dimensão, de variedade diferenciáveis e da topologia final. Isso nos permite dar uma descrição bem construída do complexo de de Rham, definindo a cohomologia como a cohomologia final com respeito a inclusão das variedades, usando então o complexo de de Rham. O leitor poderá observar que fazemos isto no apêndice B. 
A partir do próximo capítulo nos focaremos em entender a ação de $G$ em $\Omega(M)$ quando $G$ age em $M$, construir as estruturas de $G^{*}$-módulos e $G^{*}$ álgebras, mostrar que $\Omega(M)$ satisfaz a definição de $G^{*}$-álgebra e estudar a cohomologia equivariante nestas categorias. 


\section{4}

\section{Identidades Diferenciais: Ação de $G$ e Derivações em $M$}

Neste capítulo estudaremos as estruturas de derivações oriundas da ação de um grupo de Lie $G$ em uma variedade $M$. Esta será a porta de entrada para estudar $G^{*}$-módulos que serão criados para generalizar as identidades, puramente geométricas, que veremos neste capítulo para um contexto absolutamente algébrico.

Antes de iniciarmos, introduziremos uma notação assim como em [GS], texto em que se baseou está dissertação. Dado uma álgebra de Lie $g$, consideremos uma base $\xi_{1}, \ldots, \xi_{n}$ (lembrando que uma álgebra é um espaço vetorial), então podemos escrever qualquer elemento de $g$ como combinação linear da base, em particular:

$$
\left[\xi_{i}, \xi_{j}\right]=\sum_{k} c_{i j}^{k} \xi_{k}
$$

Mas para simplificar a notação escreveremos apenas $\left[\xi_{i}, \xi_{j}\right]=c_{i j}^{k} \xi_{k}$. Os termos $c_{i j}^{k}$ são chamados as constantes estruturais relativas a base escolhida de $g$.

Consideremos novamente $M$ uma variedade diferenciável e, agora, $G$ apenas um grupo de Lie que age em $M$. Considere também $g$ a álgebra de Lie de $G$.

Dada a ação de $G$ em $M$, para todo $a \in G$ temos uma função suave de $M$ em $M$

$$
\phi_{a}: M \rightarrow M
$$

Repare ainda que pelas propriedades de ação temos que

$$
\phi_{a} \circ \phi_{b}=\phi_{a b}
$$

Como vimos no capítulo 2, um mapa no nível das variedades nos dá um mapa no nível das formas dado pelo pullback. Sendo assim, podemos definir uma representação $\rho$ de $G$ no complexo de de Rham de $M$. Tome $\omega \in \Omega(M)$ e $a \in G$. Definimos

$$
\rho_{a} \omega:=\left(\phi_{a}^{-1}\right)^{*} \omega
$$

e escreveremos em geral aw em vez de $\rho_{a} \omega$. 
Agora, considere $\xi \in g$. $\xi$ define um subgrupo a um parâmetro de $G$, $t \rightarrow \exp t \xi$. E portanto define uma ação de $g$ em $M$ dado pela transformação a um parâmetro. Podemos considerar com isso, o gerador infinitesimal de transformações a um parâmetro (um campo vetorial em $M$ ) definido como

$$
\xi_{M}^{+}(x):=\frac{d}{d t}\left(\left.(\exp t \xi)(x)\right|_{t=0}\right.
$$

A derivada de Lie gera uma ação infinitesimal muito interessante. Mas no nosso caso, como estamos trabalhando com campos invariantes, a álgebra de Lie de $G$, o fluxo que iremos pegar é dado pela exponêncial. Dessa forma, definimos

$$
\mathcal{L}_{\xi} \omega:=\left.\frac{d}{d t}\left(\rho_{\exp t \xi} \omega\right)\right|_{t=0}=\left.\frac{d}{d t}\left((\exp -t \xi)^{*} \omega\right)\right|_{t=0}
$$

onde $\xi \in g$. Repare que

$$
\begin{gathered}
\mathcal{L}_{\xi}(\omega \nu)=\left.\frac{d}{d t}\left((\exp -t \xi)^{*} \omega \nu\right)\right|_{t=0}= \\
=\left.\frac{d}{d t}\left((\exp -t \xi)^{*} \omega\right)\right|_{t=0} \cdot \nu+\left.\omega \cdot \frac{d}{d t}\left((\exp -t \xi)^{*} \nu\right)\right|_{t=0}= \\
=\left(\mathcal{L}_{\xi} \omega\right) \nu+\omega\left(\mathcal{L}_{\xi} \nu\right) .
\end{gathered}
$$

Logo o operador $\mathcal{L}_{\xi}: \Omega(M) \rightarrow \Omega(M)$ é uma derivação. Precisamente, uma derivação de grau zero, já que, não altera o grau da forma.

Podemos também pensar em $\mathcal{L}_{\xi}$ através do gerador infinitesimal da transformação a um parâmetro, isto é, como a representação de $\rho_{a} \omega$ é dado por $\left(\phi_{a}^{-1}\right)^{*} \omega$, para obter uma ação de $g$ em $\Omega(M)$, consideraremos a derivação de Lie em relação ao gerador infinitesimal do grupo a um parâmetro $t \mapsto \exp (-t \xi)$ (para representar os campos invariantes pela direita), que denotaremos por $\xi_{M}^{-}$. Repare que $\xi_{M}^{-}=-\xi_{M}^{+}$.

Ou seja, $\mathcal{L}_{\xi}$ é a derivada de Lie do campo vetorial correspondente a $\xi \in M$, isto é, a derivada de Lie em relação ao campo $\xi_{M}^{-}$.

Também, sobre este campo, podemos definir uma outra derivação em $\Omega(M)$, chamada de produto interior. Defina o operador

$$
\iota: \Omega^{k}(M) \rightarrow \Omega^{k-1}(M)
$$


de modo que, para $\chi_{1}, \ldots, \chi_{k-1}$ campos vetoriais em $M$,

$$
\left(\iota_{\xi} \omega\right)_{p}\left(\chi_{1}, \ldots, \chi_{k-1}\right)=\omega\left(\xi_{M}^{-}, \chi_{1}, \ldots, \chi_{k-1}\right)
$$

Como já falado, $\iota_{\xi}$ é uma derivação, em outros termos, por [Tu],

$$
\iota_{\xi}(\omega \nu)=\left(\iota_{\xi} \omega\right) \nu+(-1)^{k} \omega\left(\iota_{\xi} \nu\right) \text { se } \omega \in \Omega^{k}(M)
$$

uma derivação ímpar de grau -1 .

Dada uma ação de $G$ em $M$ e $\xi \in g$ temos então três derivações, $d, \mathcal{L}_{\xi}, \iota_{\xi}$. Vejamos então algumas identidades fundamentais envolvendo tais derivações. Essas equações são chamadas de equações de Weil.

Considerando $\xi_{1}, \ldots, \xi_{n}$ uma base de $g, a \in G$, e por uma notação simplificada, escrevendo $\mathcal{L}_{i}=\mathcal{L}_{\xi_{i}}$ e $\iota_{i}=\iota_{\xi_{i}}$, temos:

Teorema 4.0.1 (Equações de Weil) As seguintes igualdades são verdadeiras:

1. $\iota_{i} \iota_{j}+\iota_{j} \iota_{i}=0$

2. $\mathcal{L}_{i} \iota_{j}-\iota_{j} \mathcal{L}_{i}=\iota_{[i, j]}=c_{i j}^{k} \iota_{k}$

3. $\mathcal{L}_{i} \mathcal{L}_{j}-\mathcal{L}_{j} \mathcal{L}_{i}=\mathcal{L}_{[i, j]}=c_{i j}^{k} \mathcal{L}_{k}$

4. $d \iota_{i}+\iota_{i} d=\mathcal{L}_{i}$

5. $d \mathcal{L}_{i}-\mathcal{L}_{i} d=0$

6. $d^{2}=0$

7. $\rho_{a} \circ \mathcal{L}_{i} \circ \rho_{a}^{-1}=\mathcal{L}_{A d(a) \xi_{i}}$

8. $\rho_{a} \circ \iota_{i} \circ \rho_{a}^{-1}=\iota_{A d(a) \xi_{i}}$

onde Ad é a representação adjunta de $G$ em g.

Prova: [das identidades fundamentais]

Nesta demonstração faremos um abuso de notação, se for preciso, escreveremos simplesmente $\xi_{i}$ em vez do gerador do campo infinitesimal. Vejamos então a prova de cada equação. 
6. É o teorema 2.1.2.

1. Dado $\omega \in \Omega^{k+2}(M)$

$$
\begin{gathered}
\iota_{i} \iota_{j} \omega\left(\chi_{1}, \ldots, \chi_{k}\right)=\omega\left(\xi_{i}, \xi_{j}, \chi_{1}, \ldots, \chi_{k}\right)=-\omega\left(\xi_{j}, \xi_{i}, \chi_{1}, \ldots, \chi_{k}\right)= \\
-\iota_{j} \iota_{i} \omega\left(\chi_{1}, \ldots, \chi_{k}\right)
\end{gathered}
$$

5.Dado $\omega \in \Omega^{k+2}(M)$

$$
\begin{gathered}
\mathcal{L}_{i} d \omega=\left.\frac{d}{d t}\left(\left(\exp -t \xi_{i}\right)^{*} d \omega\right)\right|_{t=0}=\left.\frac{d}{d t}\left(d\left(\exp -t \xi_{i}\right)^{*} \omega\right)\right|_{t=0}= \\
\left.d\left(\frac{d}{d t}\left(\exp -t \xi_{i}\right)^{*} \omega\right)\right|_{t=0}=d \mathcal{L}_{i} \omega
\end{gathered}
$$

4. Esta equação é também conhecida como a fórmula mágica de Cartan.

Podemos reduzir a um problema local. Por 5., $\mathcal{L}_{i}$ comuta com $d$. Além disso, como $\iota_{i}$ e $d$ são derivações ímpares, $\log 0\left(d \iota_{i}+\iota_{i} d\right)$ é uma derivação par, que, claramente, comuta com $d$. Assim, se o resultado vale para $\omega$ e $\nu$, pela definição de $\mathcal{L}_{i}$, vale para $\omega \nu$ e, pela comutatividade, vale para $d \omega$. Logo, é suficiente mostrar para $f \in \Omega^{0}(M)$.

$$
\left(d \iota_{i}+\iota_{i} d\right) f=\iota_{i} d f=(d f)\left(\xi_{i}\right)=\xi_{i} f=\mathcal{L}_{i} f
$$

2. Em [Tu] "Loring Tu"nos dá a fórmula global para derivada de Lie que diz que

$$
\left(\mathcal{L}_{i} \omega\right)\left(\chi_{1}, \ldots, \chi_{k}\right)=\xi_{i}\left(\omega\left(\chi_{1}, \ldots, \chi_{k}\right)\right)-\sum_{j=1}^{k} \omega\left(\chi_{1}, \ldots,\left[\xi_{i}, \chi_{j}\right], \ldots, \chi_{k}\right)
$$

Logo

$$
\begin{gathered}
\left(\iota_{j} \mathcal{L}_{i} \omega\right)\left(\chi_{1}, \ldots, \chi_{k-1}\right)= \\
\xi_{i}\left(\omega\left(\xi_{j}, \chi_{1}, \ldots, \chi_{k-1}\right)\right)-\omega\left(\left[\xi_{i}, \xi_{j}\right], \chi_{1}, \ldots, \chi_{k-1}\right)-\sum_{l=1}^{k-1} \omega\left(\xi_{j}, \ldots,\left[\xi_{l}, \chi_{l}\right], \ldots, \chi_{k-1}\right)= \\
\xi_{i}\left(\iota_{j} \omega\left(\chi_{1}, \ldots, \chi_{k-1}\right)\right)-\iota_{\left[\xi_{i}, \xi_{j}\right]} \omega\left(\chi_{1}, \ldots, \chi_{k-1}\right)-\sum_{l=1}^{k-1} \iota_{j} \omega\left(\chi_{1}, \ldots,\left[\xi_{i}, \chi_{l}\right], \ldots, \chi_{k-1}\right)= \\
\left(\mathcal{L}_{i} \iota_{j} \omega\right)\left(\chi_{1}, \ldots, \chi_{k-1}\right)-\iota_{\left[\xi_{i}, \xi_{j}\right]} \omega\left(\chi_{1}, \ldots, \chi_{k-1}\right)
\end{gathered}
$$

3. Por 4. temos que

$$
d \iota_{[i, j]}+\iota_{[i, j]} d=\mathcal{L}_{[i, j]},
$$


mas por 5. temos que

$$
d \iota_{[i, j]}+\iota_{[i, j]} d=\mathcal{L}_{i} d \iota_{j}-d \iota_{j} \mathcal{L}_{i}+\mathcal{L}_{i} \iota_{j} d-\iota_{j} d \mathcal{L}_{i}
$$

e novamente, por 4., é igual a

$$
\mathcal{L}_{i}\left(-\iota_{j} d+\mathcal{L}_{j}\right)-\left(-\iota_{j} d+\mathcal{L}_{j}\right) \mathcal{L}_{i}+\mathcal{L}_{i} \iota_{j} d-\iota_{j} d \mathcal{L}_{i}=\mathcal{L}_{i} \mathcal{L}_{j}-\mathcal{L}_{j} \mathcal{L}_{i}
$$

7. Dado $\omega \in \Omega(M)$, temos que

$$
\begin{aligned}
& \left(\rho_{a} \circ \mathcal{L}_{i} \circ \rho_{a}^{-1}\right)(\omega)=\left.\left(\phi_{a}^{-1}\right)^{*} \frac{d}{d t}\left(\left(\left(\exp -t \xi_{i}\right) \circ \phi_{a^{-1}}^{-1}\right)^{*} \omega\right)\right|_{t=0}= \\
& \left.\frac{d}{d t}\left(\left(a \circ\left(\exp -t \xi_{i}\right) \circ a^{-1}\right)^{*} \omega\right)\right|_{t=0}=\left.\frac{d}{d t}\left(\left(c_{a}\left(\exp -t \xi_{i}\right)\right)^{*} \omega\right)\right|_{t=0}
\end{aligned}
$$

onde $c_{a}$ é como no capítulo 2, isto é, $A d(a)=d\left(c_{a}\right)_{e}$. Assim, por [Mar] temos que se $\psi: G \rightarrow G^{\prime}$ é um homomorfismo de grupos e $\theta \in g, \psi(\exp (\theta))=$ $\exp \left(d \psi_{e}(\theta)\right)$. Dessa forma, fazendo $\psi=c_{a}$ concluímos que

$$
\left.\frac{d}{d t}\left(\left(c_{a}\left(\exp -t \xi_{i}\right)\right)^{*} \omega\right)\right|_{t=0}=\frac{d}{d t}\left(\left.(\exp -t(A d(a) \xi) \omega)\right|_{t=0}=\mathcal{L}_{A d(a) \xi_{i}} .\right.
$$

8. Por 4.

$$
\mathcal{L}_{A d(a) \xi_{i}}=d \iota_{A d(a) \xi_{i}}+\iota_{A d(a) \xi_{i}} d
$$

Mas por 7. e 4., novamente

$$
\mathcal{L}_{A d(a) \xi_{i}}=\left(\rho_{a} \circ \mathcal{L}_{i} \circ \rho_{a}^{-1}\right)(\omega)=\rho_{a} d \iota_{i} \rho_{a^{-1}}+\rho_{a} \iota_{i} d \rho_{a^{-1}}
$$

E como $\rho_{a}$ comuta com $d$, pois é dado pelo pullback de $\phi_{a}^{-1}$, temos, por 4 . que

$$
\rho_{a} \circ \iota_{i} \circ \rho_{a}^{-1}=\iota_{A d(a) \xi_{i}}
$$

Temos com isto uma caracterização bem geométrica da ação de $G$ em $M$. Iremos, no próximo capítulo, estender esta construção em um ponto de vista mais algébrico, dando uma ideia mais geral de cohomologia equivariante, que não considera apenas uma variedade $M$. Desta forma, trabalharemos de forma puramente algébrica, nos permitindo, por exemplo, usar a álgebra de Weil e o modelo de Cartan, como veremos nos capítulos 6 e 7 . 


\section{5 \\ $G^{*}$-módulos}

Como falado no capítulo anterior, neste iremos generalizar e algebrizar algumas ideias presente no anterior, tentando estender as equações de Weil a mais espaços, afim de gerar uma categoria mais geral. Iremos introduzir a teoria equivariante de $G^{*}$-módulos e definir a cohomologia equivariante de $G^{*}$-módulos.

Uma observação que devemos fazer aqui é que usaremos muitos elementos da teoria de álgebra de Lie. Chamo atenção para isto, pois algumas estruturas álgébricas, assim como o cálculo delas, necessitam que trabalhemos com um corpo algébricamente fechado e de característica 0, um exemplo disto é a álgebra de Cartan e, consequentemente, grupo de Catran. Desta forma, diferente do começo onde trabalhamos sobre o corpo dos reais, $\mathbb{R}$, usaremos $\mathbb{C}$, os complexos.

Para começar a entender a estrutura que dá nome a este capítulo, $G^{*}$ módulos, temos que entender o que Guillemin e Sternberg em [GS] chamam de "super"na linguagem matemática.

Basicamente, o termo "super"representa uma graduação na estrutura em que está trabalhando, no nosso caso, espaços vetoriais e ágebras. A graduação é dada por $\mathbb{Z} / 2 \mathbb{Z}=\{0,1\}$ e assim, um super espaço vetorial $V$, é um espaço vetorial que pode ser escrito como a soma

$$
V=V_{0} \oplus V_{1}
$$

de espaços vetoriais. Com uma nomenclatura óbvia, um elemento $v$ é dito par se está em $V_{0}$ e ímpar se esta em $V_{1}$.

Os espaços que estaremos trabalhando, em geral, possui uma $\mathbb{Z}$ graduação que pode ser pensada como uma $\mathbb{Z} / 2 \mathbb{Z}$-graduação de forma trivial. 
Considere

$$
V=\underset{i \in \mathbb{Z}}{\oplus} V_{i}
$$

Podemos definir

$$
V_{0}=\bigoplus_{i \in \mathbb{Z}} V_{2 i}, V_{1}=\bigoplus_{i \in \mathbb{Z}} V_{2 i+1} .
$$

e temos a estrutura de super espaço vetorial.

A estrutura de super álgebra segue as mesmas condições, a única hipótese adicional é que o produto tem que preservar a graduação, no sentido de que se $S$ é uma super álgebra, então

$$
S_{i} \cdot S_{j} \subset S_{i+j}
$$

Um exemplo clássico desta estrutura é o espaço dos endomorfismos de um espaço vetorial $V$, ou simplesmente $\operatorname{End}(V)$. Consideramos o produto como a composição e a graduação como sendo

$$
\bigoplus_{i \in \mathbb{Z}} \operatorname{End}_{i}(V)
$$

onde $\operatorname{End}_{i}=\left\{\gamma \in \operatorname{End}(V) \mid \gamma: V_{k} \rightarrow V_{k+i}\right\}$. É claro que $\operatorname{End}(V)$ satisfaz a hipótese do produto na super álgebra, pois se $\gamma \in \operatorname{End}_{i}(V)$ e $\alpha \in \operatorname{End}_{j}(V)$ então

$$
\alpha \circ \gamma: V_{k} \rightarrow V_{k+i+j}
$$

pois $\gamma: V_{k} \rightarrow V_{k+i}$ e $\alpha: V_{k+i} \rightarrow V_{k+i+j}$. Assim, $\operatorname{End}(V)$ é um exemplo de super álgebra.

Classicamente, um exemplo de álgebra de Lie interessante é dado pelo espaço dos endomorfismos de um espaço vetorial junto com o colchete de Lie, como a composição é associativa, o colchete de Lie definido é simplesmente o comutador. No contexto que estamos trabalhando, também temos uma estrutura de super comutador. Dado $\gamma \in \operatorname{End}_{i}(V)$ e $\alpha \in \operatorname{End}_{j}(V)$, o super comutador é definido como

$$
[\gamma, \alpha]:=\gamma \circ \alpha-(-1)^{i j} \alpha \circ \gamma
$$

Esta definição se estende para qualquer super álgebra associativa. Desta forma, uma super álgebra é super comutativa se seu super comutador é equivalentemente zero. Um exemplo deste caso é o nosso $\Omega(M)$.

Uma super álgera de Lie se define a partir de uma super anticomutativi- 
dade e uma super versão da identidade de Jacobi. Ou seja, se

$$
g=\underset{i \in \mathbb{Z}}{\oplus} g_{i}
$$

é uma super álgebra de Lie, então, para todo $u \in g_{i}, v \in g_{j}$ e $w \in g$, temos

$$
\begin{aligned}
& -[u, v]+(-1)^{i j}[v, u]=0 \\
& -[u,[v, w]]=[[u, v], w]+(-1)^{i j}[v,[u, w]] .
\end{aligned}
$$

O exemplo interessante neste caso e que será muito importante é uma visão algébrica da construção geométrica que fizemos no capítulo anterior. Considere $g$ uma álgebra de Lie e $\xi_{1}, \ldots, \xi_{n}$ base de $g$. Definimos então a super álgebra de Lie $\bar{g}$ como

$$
\bar{g}=g_{-1} \oplus g_{0} \oplus g_{1}
$$

onde os outros graus da graduação são $0, g_{-1}$ é um espaço vetorial de base $\iota_{1}, \ldots, \iota_{n}, g_{0}$ é um espaço vetorial com base $\mathcal{L}_{1}, \ldots \mathcal{L}_{n}$ e $g_{1}$ é um espaço vetorial gerado por $d$. Repare que, como espaços vetoriais, $g_{-1}$ e $g_{0}$ são isomorfos a $g$. Definimos então o colchete para satisfazer as igualdades de 1 a 6 das equações de Weil, isto é,

$$
\begin{gathered}
{\left[\iota_{i}, \iota_{j}\right]=0} \\
{\left[\mathcal{L}_{i}, \iota_{j}\right]=c_{i j}^{k} \iota_{k}} \\
{\left[\mathcal{L}_{i}, \mathcal{L}_{j}\right]=c_{i j}^{k} \mathcal{L}_{k}} \\
{\left[d, \iota_{i}\right]=\mathcal{L}_{i}} \\
{\left[d, \mathcal{L}_{i}\right]=0} \\
{[d, d]=0 .}
\end{gathered}
$$

Desta forma, podemos ver que $\left[g_{1}, g_{1}\right]=0,\left[g_{-1}, g_{-1}\right]=0,\left[g_{0}, g_{0}\right] \subset g_{0}$, $\left[g_{-1}, g_{1}\right] \subset g_{0},\left[g_{0}, g_{1}\right] \subset g_{1}$ e, por fim, $\left[g_{0}, g_{-1}\right] \subset g_{-1}$. Logo satisfaz as condições de uma super álgebra. Também não é difícil ver que satisfaz as condições de uma super álgebra de Lie.

Uma derivação $D$ em uma super álgebra $S$ é um elemento contido em $\operatorname{Der}(S) \subset \operatorname{End}(S)$ onde $\operatorname{Der}_{k}(S) \subset \operatorname{End}_{k}(S)$ e para todo $D^{*} \in \operatorname{Der}_{k}$,

$$
D^{*}(u v)=\left(D^{*} u\right) v+(-1)^{k j} u\left(D^{*} v\right)
$$


onde $u \in S_{j}$ e por $u v$ estamos considerando o produto, na super álgebra $S$, de $u$ por $v$. Neste caso, dizemos que $D^{*}$ é uma derivação de grau $k$. Se $k$ é par dizemos que é uma derivação de grau par e caso o contrário, dizemos que $D^{*}$ é uma derivação de grau ímpar. Repare que o mapa $v \rightarrow[u, v]$ satisfaz as hipóteses graças a identidade de Jacobi, então, assim como na teoria de álgebras de Lie, o colchete por um elemento é uma derivação.

Outra observação que podemos fazer é que graças as construções geométricas que fizemos no capítulo anterior, temos uma consequência algébrica, no sentido que podemos dizer que $\bar{g}$ age por derivação em $\Omega(M)$ se $G$ age em $M$.

Agora, podemos fazer algumas considerações sobre derivações. A primeira é que a ação da derivação é determinada no sistema de geradores. Neste sentido, se duas derivações concordam no sistema de geradores então elas concordam sempre.

Outra consideração é que o corpo de escalares está em $S_{0}$ e se $D$ é uma derivação, e $1 \in S_{0}$, então $D(1)=D\left(1^{2}\right)=2 D(1)=0$. Além disso, assim como na teoria de álgebra de Lie, DerS é uma subálgebra de Lie de EndS. A prova não é muito difícil e pode ser vista diretamente nos geradores. Em particular, se $D_{1}$ e $D_{2}$ são derivações de grau ímpar, então

$$
\left[D_{1}, D_{2}\right](u v)=\left(D_{1} D_{2}+D_{2} D_{1}\right)(u v)=\left(\left[D_{1}, D_{2}\right] u\right) v+u\left[D_{1}, D_{2}\right] v
$$

ou seja, o comutador de duas derivações ímpares é uma derivação de grau par. Por fim, se $D$ é ímpar, basta verificar nos geradores para ver que $D^{2}=0$.

Retornando às super álgebras, em uma álgebra não graduada pode ser dada uma estrutura de super ágebra ao declarar que todos os seus elemento são pares e que não existem elementos de grau ímpar diferente de zero. Sobre o mesmo efeito, consideremos uma álgebra graduada qualquer. A princípio ela não necessariamente satisfaz a propriedade do produto de super álgebra. Entretando isto pode ser resolvido duplicando os graus originais de cada elemento. Caso a álgebra original fosse comutativa, a super álgera será super comutativa.

Um exemplo importante e que será utilizada no futuro de super álgebra, como acima, é a álgebra simétrica $S(V)$. Ela será importate no estudo da álgebra de Weil. $S(V)$, como o nome já diz, é uma estrutura de álgebra onde 
o produto é comutativo, isto é,

$$
S^{n}(V)=\frac{\left\{v_{1} \otimes \ldots \otimes v_{n} \mid v_{1}, \ldots v_{n} \in V\right\}}{v_{1} \otimes \ldots \otimes v_{n} \sim v_{\sigma(1)} \otimes \ldots \otimes v_{\sigma(n)}}
$$

onde $\sigma \in S_{n}, S_{n}$ o grupo simétrico. Podemos pensar um elemento em $S^{n}(V)$ como um polinômio homogêneo de grau $n$ em $V^{*}$. E como foi dito antes, consideraremos este polinômio de grau $n$ como tendo grau $2 n$ em $S(V)$.

Uma construção importante de super álgebras é dado pelo produto tensorial. Sejam $A$ e $B$ super álgebras, definimos o produto em $A \otimes B$ por

$$
\left(a_{1} \otimes b_{1}\right) \cdot\left(a_{2} \otimes b_{2}\right)=(-1)^{i j} a_{1} a_{2} \otimes b_{1} b_{2}
$$

onde $\operatorname{deg} a_{2}=i$ e $\operatorname{deg} b_{1}=j$. Por essa definição, se as duas são álgebras comutativas, o produto tensorial também será. Além disso, essa é a única definição de produto tensorial para o qual os mapas

$$
\begin{array}{ll}
A \rightarrow A \otimes B, & a \mapsto a \otimes 1 \\
B \rightarrow A \otimes B, & b \mapsto 1 \otimes b
\end{array}
$$

são morfismos de álgebra e

$$
(a \otimes 1) \cdot(1 \otimes b)=a \otimes b
$$

Esta ideia é interessante pois pode nos fornecer relações importantes entre super álgebras. Por exemplo, considere $V$ e $W$ espaços vetoriais. Temos na categoria de super álgebra que $\wedge(V \oplus W)=\wedge(V) \otimes \wedge(W)$, pois podemos escolher uma base de $V \oplus W e_{1}, \ldots, e_{n}, f_{1}, \ldots f_{m} \operatorname{com} e_{i} \in V$ e $f_{j} \in W$. Assim, temos que elementos da forma

$$
e_{i 1} \wedge \ldots \wedge e_{i k} \wedge f_{j 1} \wedge \ldots \wedge f_{j l}
$$

formam uma base de $\wedge(V \oplus W)$. Um outro exemplo é que $\Omega(M) \otimes \Omega(N)$ é uma subálgebra de $\Omega(M \times N)$, mas esta relação é mais fácil de ver a partir do pullback das projeções.

E ainda, se $V$ e $W$ são super espaços vetoriais, então podemos considerar $\operatorname{End}(V) \otimes \operatorname{End}(W)$ como um subespaço de $\operatorname{End}(V \otimes W)$ de acordo com a regra

$$
\left(a_{1} \otimes b_{1}\right) \cdot\left(a_{2} \otimes b_{2}\right)=(-1)^{i j} a_{1} a_{2} \otimes b_{1} b_{2}
$$


se $\operatorname{deg} b_{1}=i$ e $\operatorname{deg} a_{2}=j$. Mas também temos que $\operatorname{End}(V) \otimes E n d(W)$ é uma subálgebra de $\operatorname{End}(V \otimes W)$, para ver isso, temos que mostrar que o produto em $\operatorname{End}(V \otimes W)$ se restringe ao produto em $\operatorname{End}(V) \otimes \operatorname{End}(W)$. Ora, mas se $\operatorname{deg} x=p, \operatorname{deg} b_{2}=q, \operatorname{deg} b_{1}=j$ e $\operatorname{deg} a_{2}=i$ temos que:

$$
\left(a_{1} \otimes b_{1}\right)\left(\left(a_{2} \otimes b_{2}\right)(x \otimes y)\right)=(-1)^{i j}(-1)^{j(p+1)} a_{1} a_{2} x \otimes b_{1} b_{2} y
$$

e

$$
\left(\left(a_{1} \otimes b_{1}\right)\left(a_{2} \otimes b_{2}\right)\right)(x \otimes y)=(-1)^{i j}(-1)^{(j+Q) p} a_{1} a_{2} x \otimes b_{1} b_{2} y .
$$

Assim temos a igualdade das duas equações e, então, como podemos observar pelo produto em $\operatorname{End}(V \otimes W)$, temos que o produto em $\operatorname{End}(V) \otimes$ $\operatorname{End}(W)$ nada mais é do que o produto em $\operatorname{End}(V \otimes W)$ na restrição. Em outras palavras, o produto em $\operatorname{End}(V \otimes W)$ quando restrito a $\operatorname{End}(V) \otimes \operatorname{End}(W)$ ainda fica em $\operatorname{End}(V) \otimes \operatorname{End}(W)$.

\section{1}

\section{$G^{*}$-módulos e $G^{*}$-álgebras}

Para começar a seção vale a pena ressaltar que o termo $G^{*}$-módulo e, consequentemente, o termo $G^{*}$-álgebra se deve a Victor W. Guillemin e Shlomo Sternberg na referência [GS], o qual tomamos como base. Entretanto, o conceito e a noção dos termos é mais antigo e se deve a Cartan em [Car], segundo os próprios Victor W. Guillemin e Shlomo Sternberg.

A ideia do conceito é generalizar o exemplo geométrico da ação de um grupo de Lie em uma variedade.

Sendo assim, considere $G$ um grupo de Lie, $g$ sua álgebra de Lie e $\bar{g}$ a super álgebra de Lie construida a partir de $g$ como feito no início do capítulo.

Definição 5.1.1 Definimos uma $G^{*}$-álgebra como uma super álgebra comutativa A junto com uma representação $\rho$ de $G$ como automorfismos de $A$ e uma ação de $\bar{g}$ como super derivações de A que satifaz, para todo a $\in G$ e $\xi \in g$, o seguinte:

$$
\begin{gathered}
\left.\frac{d}{d t} \rho(\exp t \xi)\right|_{t=0}=\mathcal{L}_{\xi} \\
\rho(a) \mathcal{L}_{\xi} \rho\left(a^{-1}\right)=\mathcal{L}_{A d_{a} \xi} \\
\rho(a) \iota_{\xi} \rho\left(a^{-1}\right)=\iota_{A d_{a} \xi} \\
\rho(a) d \rho\left(a^{-1}\right)=d
\end{gathered}
$$


E finalmente a definição de $G^{*}$-módulo:

Definição 5.1.2 Definimos um $G^{*}$-módulo como um super espaço vetorial A junto com uma representação linear $\rho$ de $G$ em $A$ e um homomorfismo $\bar{g} \rightarrow \operatorname{End}(A)$ que satisfaz as equações 5.1.1, 5.1.2, 5.1.3 e 5.1.4.

Perceba então que $\bar{g}$ não precisa agir como derivação em $A$ e $G$ como um automorfismo, o que é menos que o necessário para uma $G^{*}$-álgebra. Todavia, podemos ver que uma $G^{*}$-álgebra é uma superálgebra comutativa que é um $G^{*}$-módulo tal que $G$ age como um automorfismo e $\bar{g}$ como derivação. Podemos observar também que uma $G^{*}$-álgebra é um $G^{*}$-módulo se esquecermos a estrutura multiplicativa.

Repare que para a equação 5.1.1 ter sentido, precisamos definir a derivação que aparece na equação. Então, para isso acontecer, $A$ deverá ter algum tipo de topologia, ou, que pelo menos todo elemento de $A$ esteja contido em subespaço finito $G$-invariante de $A$ como em [GS] (admitindo assim uma estrutura de espaço vetorial topológico na restrição). Exemplos importantes para cada uma das opções é $\Omega(M)$ e $S\left(g^{*}\right)$ (a álgebra simétrica do dual de $g$ ) respectivamente.

Vale acrescentar que se $G$ é conexo, segundo os próprios Victor W. Guillemin e Shlomo Sternberg as equações 5.1.2, 5.1.3 e 5.1.4 são consequências diretas da equação 5.1.1. Isto se dá essencialmente pois todo elemento da componente conexa da identidade é imagem de um subgrupo a um parâmetro de $G$, ou seja, dado $a \in G$, existe $\xi \in g$ tal que $a=\exp t \xi$. Assim, graças a equação 5.1.1 temos que:

$$
\begin{gathered}
\frac{d}{d t} \rho(\exp t \xi)=\left.\frac{d}{d s} \rho(\exp (t+s) \xi)\right|_{s=0}=\left.\frac{d}{d s}(\rho(\exp t \xi \circ \exp s \xi))\right|_{s=0}= \\
\left.\frac{d}{d s} \rho(\exp t \xi) \circ \rho(\exp s \xi)\right|_{s=0}=\left.\rho(\exp t \xi) \circ \frac{d}{d s} \rho(\exp s \xi)\right|_{s=0}=\rho(\exp t \xi) \circ \mathcal{L}_{\xi}
\end{gathered}
$$

Mostremos então, por exemplo, que temos como consequência a 5.1.3.

$$
\begin{gathered}
\frac{d}{d t}\left[\rho(\exp t \xi) \circ \iota_{A d(\exp -t \xi) \zeta} \circ \rho(\exp -t \xi)\right]= \\
\rho(\exp t \xi) \circ\left(\left[\mathcal{L}_{\xi}, \iota_{A d(\exp -t \xi) \zeta}\right]-\iota_{[\xi, A d(\exp -t \xi) \zeta]}\right) \circ \rho(\exp -t \xi)=0
\end{gathered}
$$

usamos na segunda linha que $g$ satisfaz a equação 5.0.2. Assim, fazendo $\nu=A d_{a^{-1}} \zeta$ temos a validade da equação 5.1.3. As outras equações são análogas. 
Após definirmos os conjuntos que serão objetos de trabalho, precisamos dos morfismos para definir a categoria.

Definição 5.1.3 Sejam $A$ e $B G^{*}$-módulos. Diremos que o mapa linear

$$
f: A \rightarrow B
$$

é um morfismo de $G^{*}$-módulos se para cada $x \in A, b \in G$ e $\xi \in g$ temos:

$$
\begin{gathered}
{[\rho(b), f]=0} \\
{\left[\mathcal{L}_{\xi}, f\right]=0} \\
{\left[\iota_{\xi}, f\right]=0} \\
{[d, f]=0}
\end{gathered}
$$

onde $[\cdot, \cdot]$ represenda o super comutador.

Se para cada $i$,

$$
f: A_{i} \rightarrow B_{i+k}
$$

$f$ é dita de grau $k$. Se $k$ é par diremos que $f$ é um morfismo par, caso contrário, $f$ é dito morfismo de grau ímpar. Caso $f$ seja par (especialmente de grau zero como geralmente iremos trabalhar) podemos escrever as equações 5.1.5, 5.1.6, 5.1 .7 e 5.1 .8 como:

$$
\begin{gathered}
\rho(a) f(x)=f(\rho(b) x) \\
\mathcal{L}_{\xi} f(x)=f\left(\mathcal{L}_{\xi} x\right) \\
\iota_{\xi} f(x)=f\left(\iota_{\xi} x\right) \\
d f(b)=f(d b) .
\end{gathered}
$$

Ou seja, $f$ preserva a $G^{*}$-ação.

Enquanto para a categoria de $G^{*}$-álgebras, se considerarmos os conjuntos $A$ e $B$ como $G^{*}$-álgebras, o mapa $f$ de $A$ a $B$ é um morfismo de $G^{*}$-álgebras se $f$ é um homomorfismo de álgebras que satisfaz as equações 5.1.5, 5.1.6, 5.1.7 e 5.1.8.

Um exemplo de $G^{*}$-álgebra é a álgebra graduada formada pelas formas de uma variedade $M, \Omega(M)$. E ainda, caso $M$ e $N$ sejam $G$-variedades e 
$F: M \rightarrow N$ seja um mapa suave $G$-equivariante, então $F^{*}: \Omega(N) \rightarrow \Omega(M)$ é um morfismo de $G^{*}$-álgebras.

\section{2}

\section{Cohomologia e Homotopias em Cadeia}

A graduação de $A$ sendo uma $G^{*}$-álgebra (ou um $G^{*}$-módulo) junto com o operador $d$, que por definição é de grau 1 e $d^{2}=0$, nos permite ver $A$ como um complexo de cocadeia e definir a cohomologia sobre $A$, sendo $d$ o operador fronteira. Assim, escrevendo simplesmente $H(A)$ em vez de $H(A, d)$, temos a cohomologia de $A$ relativa a $d$. Repare que se $A=\Omega(M)$, a cohomologia de $A$, pelo teorema de de Rham, é simplesmente a cohomologia de de Rham de $M, H^{*}(M)$.

Podemos fazer algumas considerações sobre a cohomologia e os morfismos. Primeiro, $H^{*}(A)$ herda uma estrutura de super espaço vetorial de $A$ e, caso $A$ seja uma super álgebra, $H^{*}(A)$ também será uma super álgebra. Além disso, dado um morfismo $f: A \rightarrow B$, se restringirmos $f$ aos cocíclos (ou elementos fechados, pela nomeclatura de formas diferenciáveis) de $A$, isto é, aos elementos de $A$ que são levados em 0 por $d$, graças a comutativida de $d$ com $f$ visto na equação 5.1.8, temos que o mapa induzido por $f$,

$$
f_{*}: H^{*}(A) \rightarrow H^{*}(B)
$$

é um morfismo de $G^{*}$-álgebras, no caso de $A$ e $B$ serem $G^{*}$-álgebras.

Outra consideração importante é que graças a equação 5.1.4, a ação de $G$ comuta com o operador $d$, desta forma, $H^{*}(A)$ também herda a estrutura de $G^{*}$-módulo. Agora, como vimos na seção anterior, todo elemento da componente conexa da identidade de $G$ pode ser visto através da translação e, portanto, dado $a$ na componente conexa da identidade de $G, a=\exp (t \xi)$ para algum $\xi \in g$. Assim, podemos ver que a componente conexa de $G$ age trivialmente em $H^{*}(A)$, pois graças a fórmula de Cartan que podemos ver na equação 5.1 .4 , se $\omega \in A$ e $d \omega=0$, então

$$
\mathcal{L}_{\xi} \omega=d \iota_{\xi} \omega
$$

ou seja, $\mathcal{L}_{\xi} \omega$ é um cobordo (ou forma exata na nomeclatura de formas diferenciáveis), pois está na imagem de $d$. Então, novamente se $f: A \rightarrow B$ 
é um morfismo, temos o morfismo induzido

$$
f_{*}: H^{*}(A) \rightarrow H^{*}(B)
$$

de $G^{*}$-módulos.

Assim como em qualquer cohomologia, existe uma definição de aciclicidade. É uma ideia que estende, por exemplo, a cohomologia de uma variedade contrátil na teoria de de Rham. Em geral, iremos trabalhar sobre o corpo dos complexos $\mathbb{C}$ e não $\mathbb{R}$ como na teoria de de Rham em geral, assim definimos:

Definição 5.2.1 Diremos que A é acíclico se

$$
H^{k}(A)= \begin{cases}\mathbb{C}, & \text { se } k=0 \\ 0, & \text { se } k \neq 0\end{cases}
$$

A definição é análoga em qualquer cohomologia, trocando apenas o operador fronteira e o corpo base em questão.

Uma pergunta clássica na teoria de topologia algébrica é: Quando dois mapas entre espaços topológicos induzem um mesmo mapa em suas cohomologias? O teorema 2.1.4 é um exemplo de resultado que se busca.

O estudo de Homotopias em Cadeia, ou simplesmente Chain Homotopies, é muito importante para a teoria de cohomologia neste sentido. No nosso contexto, definiremos homotopias em cadeia da seguinte forma:

Definição 5.2.2 Seja $A$ e $B G^{*}$-módulos. Diremos que o mapa linear

$$
Q: A \rightarrow B
$$

é uma homotopia em cadeia se ele é impar, G-equivariante e satisfaz a igualdade

$$
\iota_{\xi} Q+Q \iota_{\xi}=0
$$

para todo $\xi \in g$.

Para ser mais preciso, como em geral assumiremos que $A$ e $B$ são $\mathbb{Z}$ graduados, exigiremos que $Q$, tenha não apenas grau ímpar, tenha grau -1 .

Repare que a $G$-equivariância implica que $Q$ comuta $\operatorname{com} \mathcal{L}_{\xi}$ para todo $\xi \in g$, desta forma temos a seguinte equação:

$$
\mathcal{L}_{\xi} Q-Q \mathcal{L}_{\xi}=0
$$


Como falamos anteriormente, uma homotopia em cadeia é utilizado para verificar quando mapas entre espaços topológicos induzem o mesmo mapa na cohomologia. Neste sentido, verifiquemos o seguinte teorema:

Teorema 5.2.3 Se $Q: A \rightarrow B$ é uma mapa em cadeia então o seguinte mapa é um morfismo de $G^{*}$-módulos:

$$
\tau:=d Q+Q d
$$

Prova: Temos que provar que as equações 5.1.5, 5.1.6, 5.1.7 e 5.1 .8 são satisfeitas para $\tau$. Ora, mas repare primeiro que $\tau=[d, Q]$ e por definição, $\left[\mathcal{L}_{\xi}, Q\right]=0$ e $\left[\iota_{\xi}, Q\right]=0$ para todo $\xi \in g$. Segue então, usando a identidade de Jacobi, que:

$$
\left[\iota_{\xi}, \tau\right]=\left[\iota_{\xi},[d, Q]\right]=\left[\left[\iota_{\xi}, d\right], Q\right]-\left[d,\left[\iota_{\xi}, Q\right]\right]=\left[\mathcal{L}_{\xi}, Q\right]-0=0
$$

Além disso, como $\left[\mathcal{L}_{\xi}, d\right]=0$ pois estamos trabalhando com $G^{*}$-módulos, temos que:

$$
\left[\mathcal{L}_{\xi}, \tau\right]=\left[\mathcal{L}_{\xi},[d, Q]\right]=\left[\left[\mathcal{L}_{\xi}, d\right], Q\right]-\left[d,\left[\mathcal{L}_{\xi}, Q\right]\right]=0-0=0
$$

Mas,

$$
[d, \tau]=[d,[d, Q]]=[[d, d], Q]-[d,[d, Q]]=-[d, \tau]
$$

$\log \mathrm{o},[d, \tau]=0$.

Por fim, como $Q$ e $d$ comutam com a ação de $G$, provamos o resultado.

Então, assim como em [Hatc], temos que o mapa induzido por $\tau$ na cohomologia é o mesmo que o mapa trivial, em outras palavras, $\tau \simeq 0$. Estendemos esta ideia para esclarecer quando dois mapas são homotópicos em cadeia e, assim, induzem o mesmo mapa em cohomologia. Isto é, se existe um mapa $Q: A \rightarrow B$ que é uma homotopia em cadeia, tal que para $\tau_{0}$ e $\tau_{1}$ morfismos de $A$ à $B$, temos que

$$
\tau_{0}-\tau_{1}=Q d+d Q
$$

então escrevemos $\tau_{0} \simeq \tau_{1}$, pois como vimos, os mapas induzidos na cohomologia são os mesmos. 
Vale a pena lembrar e resaltar como se dá os mapas de homotopia em cadeia na teoria de de Rham. Sejam $M$ e $N$ variedades diferenciáveis. Considere também os mapas $C^{\infty}, \phi_{0}: M \rightarrow N$ e $\phi_{1}: M \rightarrow N$. Como já vimos, estes mapas induzem mapas, através do pullback, no sentido oposto no nível de formas diferenciáveis.

$$
\phi_{0}^{*}: \Omega(N) \rightarrow \Omega(M) \text { e } \phi_{1}^{*}: \Omega(N) \rightarrow \Omega(M)
$$

Consideramos que, $\phi_{0}$ e $\phi_{1}$ são homotópicos suavemente se existir um mapa suave

$$
\phi: M \times I \rightarrow N
$$

onde $I$ é o intervalo $[0,1], \phi_{0}=\phi(\cdot, 0)$ e $\phi_{1}=\phi(\cdot, 1)$. Vale ressaltar que, por $[\mathrm{BT}]$, mapas contínuos entre variedades diferenciáveis são continuamente homotópicos a mapas suaves.

Teorema 5.2.4 Sobre as condições acima, $\phi_{0}^{*}$ e $\phi_{1}^{*}$ são homotópicos em cadeia. O que também prova o teorema 2.1.4.

Prova: Como de forma clássica, para cada $t \in I$, defina $\phi_{t}: M \rightarrow N$ tal que $\phi_{t}(x)=\phi(x, t)$. Isto estabelece mapas suaves para cada $t$ de $M$ a $N$, o que nos permite definir o mapa

$$
\xi_{t}: M \rightarrow T N
$$

de forma natural, isto é, para todo $x \in M, \xi_{t}(x)$ é o vetor tangente da curva $s \mapsto \phi(x, s)$ em que $s=t$. Ou seja, o mapa dado quando fixamos $w$ e verificamos a diferencial em $t$.

Agora, dado $\sigma \in \Omega^{k+1}(N)$, defina $\phi_{t}^{*}\left(\iota_{\xi_{t}} \sigma\right) \in \Omega^{K}(M)$ de forma que

$$
\phi_{t}^{*}\left(\iota_{\xi_{t}} \sigma\right)\left(\eta_{1}, \ldots, \eta_{k}\right)=\sigma\left(\xi_{t}(x), d \phi_{t}\left(\eta_{1}\right), \ldots, d \phi_{t}\left(\eta_{k}\right)\right)
$$

onde $\eta_{1}, \ldots, \eta_{k} \in T_{x} M$. Um resultado que tiramos desta equação é que:

$$
\frac{d}{d t} \phi_{t}^{*} \sigma=\phi_{t}^{*}\left(\iota_{\xi_{t}} d \sigma\right)+d \phi_{t}^{*}\left(\iota_{\xi_{t}} \sigma\right) .
$$

Infelizmente, no nosso caso, o vetor tangente depende de $t$ e, portanto, a demonstração não é tão simples como usar a definição da derivada de Lie e a fórmula mágica de Cartan. Mas, fazendo uso da identidade

$$
\frac{d}{d t} \phi_{t}^{*} \sigma=\phi_{t}^{*} \frac{d \sigma}{d t}+\phi_{t}^{*}\left(\iota_{\xi_{t}} d \sigma\right)+d \phi_{t}^{*} \iota_{\xi_{t}} \sigma
$$


que aparece em um outro livro de Victor W. Guillemin e Shlomo Stemberg, [GS2], temos que como, no nosso caso, $\sigma$ não depende de $t$, o resultado enunciado anteriormente é verificado.

Definindo então o mapa $Q: \Omega(N) \rightarrow \Omega(M)$ de forma que

$$
Q \sigma:=\int_{0}^{1} \phi_{t}^{*}\left(\iota_{\xi_{t}} \sigma\right) d t
$$

temos então que

$$
\begin{gathered}
(Q d+d Q) \sigma=\int_{0}^{1} \phi_{t}^{*}\left(\iota_{\xi_{t}} d \sigma\right) d t+d \int_{0}^{1} \phi_{t}^{*}\left(\iota_{\xi_{t}} \sigma\right) d t=\int_{0}^{1} \phi_{t}^{*}\left(\iota_{\xi_{t}} d \sigma\right)+d \phi_{t}^{*}\left(\iota_{\xi_{t}} \sigma\right) d t= \\
=\int_{0}^{1} \frac{d}{d t} \phi_{t}^{*} \sigma d t=\phi_{1}^{*}-\phi_{0}^{*} \\
(Q d+d Q) \sigma=\phi_{1}^{*}-\phi_{0}^{*}
\end{gathered}
$$

Logo o teorema é verificado.

É claro que na demonstração acima não estamos usando nenhuma estrutura de $G$-equivariância. Porém, se considerarmos $M$ e $N G$-variedades e $\phi_{0}, \phi_{1}$ e $\phi$ mapas $G$-equivariantes, como já vimos, $\Omega(M)$ e $\Omega(N)$ serão $G^{*}$ módulos e os mapas $\phi_{0}^{*}$ e $\phi_{1}^{*}$ serão $G^{*}$-morfismos. Segue então da construção que o mapa $Q$ é $G$-equivariante e satisfaz a equação 5.2.1, desta forma, temos $Q$ como uma homotopia de cadeias segunido a nossa definição original.

Definição 5.2.5 Sejam $A$ e $B G^{*}$-álgebras e $\phi: A \rightarrow B$ um homomorfismo de álgebras que é um $G^{*}$-morfismo. Dizemos que $Q: A \rightarrow B$ é uma homotopia em cadeia relativa a $\phi$, ou simplesmente uma $\phi$-homotopia se $Q$ satisfaz a equação 5.2 .1 e, para todo $x \in A_{i}, y \in A$, temos que

$$
Q(x y)=Q(x) \phi(y)+(-1)^{i} \phi(x) Q(y)
$$

Com esta definição, garantimos que $Q$ seja inteiramente determinado pelos geradores de $A$. Em contra partida, se temos o mapa $\phi$ e $Q$ satisfaz as equações 5.2.1 e 5.2.6 nos geradores de $A$, o seguinte é verificado para todo $x \in A_{i}$ e $y \in A$ :

1. $\iota_{\xi} Q(x y)=\left(\iota_{\xi}\right) \phi(y)-(-1)^{i} Q(x) \phi\left(\iota_{\xi} y\right)+(-1)^{i}\left(\iota_{\xi} \phi(x) Q(y)+\phi(x) \iota_{x i} Q(y)\right.$

2. $Q\left(\iota_{\xi}(x y)\right)=Q\left(\iota_{\xi} x\right) \phi(y)-(-1)^{i} \phi\left(\iota_{x i} x\right) Q(y)+(-1)^{i} Q(x) \phi\left(\iota_{\xi} y\right)+$ $\phi(x) Q\left(\iota_{\xi} y\right)$. 
Logo, subtraindo 1 e 2, temos que a equação 5.2.1 também é verificada para o produto e, portanto, para todos os elementos de $A$. Então, $Q$ é uma homotopia em cadeia.

\section{3}

\section{Ação Livre e a Condição (C)}

A ação de $G$ em $M$ ser livre é muito importante em alguns resultados, até pela representação de alguns termos que utilizaremos. Como já comentamos, estamos generalizando conceitos topológicos e, como vimos na construção da cohomologia equivariante topológica, a ação de $G$ ser livre é determinante para a teoria.

Porém, com base apenas na teoria de de Rham, não é tão simples verificar se uma ação é livre ou não. Precisamos então, de alguma forma, conseguir traduzir a ação de $G$ ser livre em uma perspectiva puramente algébrica, para que possamos continuar a teoria equivariante de $G^{*}$-módulos. Assim, o que faremos é diminuir a condição de ser livre, de forma a conseguirmos detectar a condição no nível infinitesimal. Neste sentimos definimos:

Definição 5.3.1 Uma ação de $G$ em $M$ é dita localmente livre se a ação infinitesimal correspondente a $g$ for livre, ou seja, se para cada $\xi \neq 0 \in g$ o campo de vetores $\xi_{M}^{-}$que gera o grupo a um parâmetro $t \mapsto \exp -t \xi$ de transformações em $M$ nunca desaparece, isto é, $\xi_{M}^{+}(p) \neq 0$ para todo $p \in M$.

Repare que, pelo que fizemos nos capítulos anterior, $G$ ser livre implica $G$ ser localmente livre. Entretanto o contrário não pode ser dito, isto é, $G$ ser localmente livre não implica $G$ ser livre. Desta forma, a classe de variedades que satisfaz a condição acima não se reduz a fibrados principais como é o caso de $G$ ser um grupo de Lie compacto e a ação de $G$ na variedade ser livre.

Seja $\xi_{1}, \ldots, \xi_{n}$ uma base de $g$. Para simplificar a notação, escreva $\chi_{i}$ em vez de $\xi_{i M}^{-}$. Assim, como a ação é localmente livre, temos que $\chi_{1}(p), \ldots, \chi_{n}(p)$ formam um conjunto linearmente independente de $T_{p} M$ para todo $p \in M$. Assim, temos que

$$
D=\underset{p \in M}{\sqcup}<\chi_{1}(p), \ldots, \chi_{n}(p)>
$$

é uma distribuição de rank $n$ em $M$, aqui pensando que a dimensão de $M$ é menor que $n$ (as considerações são imediatas se a dimensão de $M$ é $n$ ). Temos que a distribuição é involutiva pela ação. Além disso, $D$ é um subfibrado 
vetorial do fibrado tangente de $M$.

Repare que, ao complentarmos $\chi_{1}, \ldots, \chi_{n}$ para uma base de seções de $T M$, podemos considerar 1-forma $\theta^{j} \in \Omega(M)$ (o dual de $\chi_{j}$ ) tal que

$$
\iota_{i} \theta^{j}=\delta_{i}^{j}
$$

Esses $\left(\theta^{j}\right)^{\prime} s$ são as chamadas formas de conexão. Para ser mais preciso, muitas vezes consideramos a forma de conexão como $\theta \in \Omega(M) \otimes g$, com $\theta=\theta^{i} \otimes \xi_{i}$, onde $\theta^{i}$ é a forma que vimos antes. Por outro lado, se tivermos uma $G$ ação em uma variedade na qual existem formas $\theta^{a}$ que satisfazem a equação 5.3.1, então a ação é localmente livre.

Temos também outras 1-formas interessantes para o nosso contexto. Dado $\omega \in \Omega^{1}(M), \omega$ é chamada de forma horizontal se para $i=1, \ldots, n$, temos que

$$
\iota_{i} \omega=0
$$

Lembrando que $\iota_{i}=\iota_{\xi_{i}}$.

Agora, considere $\Xi$ uma métrica Riemaniana, sobre esta métrica podemos escrever $T M=D \oplus D^{\perp}$, em outras palavras, estamos completando $T M$ através de uma métrica Riemaniana, fazendo $D^{\perp}$ ser um subfibrado de $T M$ ortogonal a $D$ através de $\Xi$. Agora, nada garante que $D^{\perp}$ é $G$-invariante. Porém, se $G$ é compacto podemos fazer um processo muito interessante. Processo este que iremos usar algumas vezes nesta dissertação, ele é chamado de "averaging", ou simplesmente, como iremos chamar, fazer a média. O cálculo constitui em usar a $G$-invariância da medida de Haar, para transformar funcionais $C^{\infty}$ ordinários (podem ser também, por exemplo, formas diferenciáveis, entre outros), no nosso caso a métrica Riemaniana, em mapas $G$-invariantes.

Considere primeiro o mapa

$$
\begin{gathered}
\Xi: T M \times T M \rightarrow \mathbb{R} \\
(\chi, \tilde{\chi}) \mapsto \Xi(\chi, \tilde{\chi}) .
\end{gathered}
$$

Induzido por este mapa, conseguimos definir o mapa $T$ que nos permite compreender a ação de $G$,

$$
T: G \times(T M \times T M) \rightarrow \mathbb{R}
$$




$$
(a,(\chi, \tilde{\chi})) \mapsto \Xi\left(A d_{a} \chi, A d_{a} \tilde{\chi}\right)
$$

Então, restrito a $(\chi, \tilde{\chi})$ temos um mapa de $G$ em $\mathbb{R}$.

$$
\left.T\right|_{G \times(\chi, \tilde{\chi})} \rightarrow \mathbb{R}
$$

Assim, tomando $\nu$ uma forma de volume invariante em $G$, podemos definir uma nova métrica como:

$$
\int_{G} \Xi\left(A d_{a} \chi, A d_{a} \tilde{\chi}\right) d \nu
$$

Afirmamos que $\int_{G} \Xi\left(A d_{a} \chi, A d_{a} \tilde{\chi}\right) d \nu$ é constante na órbita de $G$. De fato, para todo $b \in G$ temos:

$$
\begin{gathered}
\left(A d_{b^{-1}}\right)^{*} \int_{G} \Xi\left(A d_{a} \chi, A d_{a} \tilde{\chi}\right) d \nu=\int_{G} A d_{b^{-1}}^{*} A d_{a^{-1}}^{*} \Xi(\chi, \tilde{\chi}) d \nu= \\
=\int_{G}\left(A d_{a^{-1}} A d_{b^{-1}}\right)^{*} \Xi(\chi, \tilde{\chi}) d \nu=\int_{G}\left(A d_{(b a)^{-1}}\right)^{*} \Xi(\chi, \tilde{\chi}) d \nu .
\end{gathered}
$$

Então, fazendo uma mudança de variáveis, $a \mapsto b a$, como a medida de Haar é invariante a translação, temos que

$$
\int_{G}\left(A d_{(b a)^{-1}}\right)^{*} \Xi(\chi, \tilde{\chi}) d \nu=\int_{G} \Xi\left(A d_{a} \chi, A d_{a} \tilde{\chi}\right) d \nu .
$$

Logo, esta nova medida é constante na órbita de $G$.

Chamemos esta nova métrica de $\Gamma$. Então, sobre $\Gamma$ podemos escrever $T M$ como a combinação $G$-invariante dada por $D \oplus D^{\perp}$. Em particular, sobre esta mesma métrica, temos que $T^{*} M=(D)^{*} \oplus\left(D^{\perp}\right)^{*}$ (onde $(D)^{*}$ é formado pelas formas anuladoras de $\left.\left(D^{\perp}\right)\right)$ é uma combinação $G$-invariante de $T^{*} M$. Denotando $(D)^{*}$ por $C$, temos então que $C$ é o subfibrado do fibrado cotangente de $M$ que é ortogonal, em relação a $\Gamma$, ao fibrado formado pelas formas horizontais ou, simplesmente, fibrado horizontal. Repare que em cada ponto, a fibra de $C$ é isomórfa a $g^{*}$.

Vale a pena ressaltar que mesmo que não consideremos $G$ compacto ou que $T^{*} M$ possa ser escrito como uma combinação $G$-invariante como acima, ainda podemos considerar $C$ como um subfibrado do fibrado cotangente, mas que agora é apenas complementar ao fibrado horizontal. Neste caso, não necessariamente, $G$-invariante. 
Observe agora que, como por definição $\iota_{b} \theta^{j}$ é constante, temos que:

$$
\mathcal{L}_{a} \iota_{b} \theta^{j}=0
$$

logo, usando a equação 5.0.2 temos

$$
\left(\left[\mathcal{L}_{a}, \iota_{b}\right]+\iota_{b} \mathcal{L}_{a}\right) \theta^{j}=c_{a c}^{j}+\iota_{b} \mathcal{L}_{a} \theta^{j}=c_{a b}^{j}+\iota_{b} \mathcal{L}_{a} \theta^{j}=0
$$

Então, conseguimos verificar como age $\mathcal{L}_{a}$ em $\theta^{j}$, isto é, como $c_{a b}^{j}+\iota_{b} \mathcal{L}_{a} \theta^{j}=0$, temos que

$$
\mathcal{L}_{a} \theta^{j}=-c_{a b}^{j} \theta^{b}+\omega_{a}^{j}
$$

onde $\omega_{a}^{j}$ é uma forma horizontal. Repare então que se $C$ é $G$-invariante, temos que $\omega_{a}^{j}=0$ e então:

$$
\mathcal{L}_{a} \theta^{j}=-c_{a b}^{j} \theta^{b}
$$

Esta é uma tradução da $G$-invariância para o nível de formas do nosso caso, basta lembrar que $\xi_{M}^{-}=-\xi_{M}^{+}, \xi_{M}^{+}(x)=\left.\frac{d}{d t}(\exp t \xi)(x)\right|_{t=0} \mathrm{e}$ $\mathcal{L}_{\xi} \beta=\left.\frac{d}{d t}\left(\rho_{\exp t \xi} \beta\right)\right|_{t=0}=\left.\frac{d}{d t}\left((\exp -t \xi)^{*} \beta\right)\right|_{t=0}$, ou seja, a $G$-invariância de $C$ é medida por $\mathcal{L}_{\xi}$ por causa da definição das curvas e, portanto, do dual das curvas.

Voltando a uma perspectiva mais algébrica afim de generalizar nossa situação, definimos:

Definição 5.3.2 Uma $G^{*}$-álgebra $A$ é dita do tipo $(\boldsymbol{C})$ se houver elementos $\theta^{i} \in A_{1}$ chamados elementos de conexão (lembrando que $A_{1}$ é referente à graduação de A) que satisfaz a equação 5.3 .1 e tal que o subespaço $C \in A_{1}$ que eles geram é invariante sobre $G$.

Em particular, repare que se $G$ é conexo a equação 5.3 .3 significa dizer que o subespaço $C$ é $G$-invariante já que a ação de $\mathcal{L}_{a}$ restrita a $C$ ainda permanece em $C$. Desta forma, se $G$ é conexo a condição $(\mathbf{C})$ pode ser substituida por $\theta^{i}$ satisfazer 5.3.3 e 5.3.1.

Outra informação importante é que em geral as propriedades de $A$ que estudaremos independem da escolha dos elementos de conexão. Agora, usando as equações 5.3.3 e 5.3.1 temos que:

$$
L_{a} \theta^{j}-d \iota a \theta^{j}=-c_{a b}^{j} \theta^{b}
$$


$\log \mathrm{O}$

$$
\iota_{a} d \theta^{j}=c_{a b}^{j} \theta^{b}
$$

Ora, mas dado $\mu^{j} 2$-forma tal que

$$
\iota_{a} \mu^{j}=0
$$

temos que

$$
\iota_{a}\left(\frac{-1}{2} c_{i k}^{j} \theta^{i} \theta^{j}+\mu^{j}\right)=-\frac{1}{2} c_{i k}^{j}\left(\iota_{a} \theta^{i} \theta^{k}-\theta^{i} \iota_{a} \theta^{k}\right)=c_{a b}^{j} \theta^{b},
$$

$\log \mathrm{O}$

$$
d \theta^{j}=\frac{-1}{2} c_{i k}^{j} \theta^{i} \theta^{k}+\mu^{j}
$$

Os elementos $\mu^{j}$ são chamados elementos de curvatura correspondentes aos elementos de conexão $\left\{\theta^{j}\right\}$.

Então, de 5.3.5 temos que

$$
d \mu^{a}=d\left(\frac{1}{2} c_{i j}^{a} \theta^{i} \theta^{j}\right)=\frac{1}{2} c_{i j}^{a}\left(d \theta^{i} \theta^{j}-\theta^{i} d \theta^{j}\right) .
$$

Mas $d \theta^{i} \theta^{j}=\left(-\frac{1}{2} c_{k l}^{i} \theta^{k} \theta^{l}+\mu^{i}\right) \theta^{j}$ e $\theta^{i} d \theta^{j}=\theta^{i}\left(-\frac{1}{2} c_{k l}^{j} \theta^{k} \theta^{l}+\mu^{j}\right)$, portanto

$$
d \theta^{i} \theta^{j}-\theta^{i} d \theta^{j}=\left(\mu^{i} \theta^{j}+\theta^{i} \mu^{j}\right)
$$

Assim, como podemos comutar $\theta^{i}$ e $\mu^{j}$, temos que

$$
d \mu^{a}=-c_{i j}^{a} \theta^{i} \mu^{j} .
$$

E, por fim, como $\mathcal{L}_{a}=\left[d, \iota_{a}\right]$, temos que $\mathcal{L}_{k} \mu^{a}=-\iota_{k} d \mu^{a}=\iota_{k} c_{i j}^{a}\left(\theta^{i} \mu^{j}\right)=$ $c_{i j}^{a} \iota_{k} \theta^{i} \mu^{j}$. Logo,

$$
\mathcal{L}_{i} \mu^{a}=-c_{i j}^{a} \mu^{j} .
$$

Uma observação natural que podemos fazer é que uma vez que tenhamos $B$ uma $G^{*}$-álgebra do tipo $(\mathbf{C})$ com elementos de conexão $\theta_{B}^{i}$, dado $A$ uma $G^{*}$-álgebra ordinária, temos que $A \otimes B$ é novamente uma $G^{*}$-álgebra do tipo (C) com elementos de conexão $1 \otimes \theta_{B}^{i}$.

Anteriormente falamos enquanto trabalhavamos com variedades que em cada ponto de $C$, o fibrado complementar ao fibrado horizontal, temos um isomorfismo natural de $g^{*}$ e a fibra no ponto. Aqui também temos uma relação semelhante. Considere $x_{1}, \ldots, x_{n}$ a base de $g^{*}$ dual a base $\xi_{1}, \ldots, \xi_{n}$. Então considere o mapa

$$
\mathcal{C}: g^{*} \rightarrow A_{1}
$$




$$
\mathcal{C}\left(x_{i}\right) \mapsto \theta^{i}
$$

Assim, o subespaço $C$ gerado pelos $\theta^{i}$ é a imagem de $g^{*}$ pelo mapa $\mathcal{C}$. Desta forma, a condição 5.3.1 pode ser escrita com base apenas em $g$ e $g^{*}$, isto é, dado $\xi \in g$ e $\theta \in g^{*}$, a equação 5.3.1 equivale a

$$
\iota_{\xi}(\mathcal{C}(\theta))=<\theta, \xi>
$$

No cálculo para construir uma métrica Riemaniana $G$-invariante usamos, sem nomear, a representação coadjunta, que nada mais é do que a representação de $G$ em $g^{*}$ contragradiente a representação adjunta. Assim como em [GS], iremos usar uma notação diferente para representar esta ação, a denotaremos como $A d^{\star}$. Ou seja, com a nova notação temos que

$$
<A d_{b}^{\star} \theta, \xi>=<\theta, A d_{b^{-1}} \xi>
$$

O interessante com respeito a esta notação é que se $\mathcal{C}$ satisfaz a equação 5.3.8 então $a \circ \mathcal{C} \circ A d_{a^{-1}}^{\star}$ também satisfaz. De fato, considerando de maneira natural que $G$ age trivialmente sobre escalares, então, graças a equação 5.1.3, temos que:

$$
\iota_{\xi}\left(a \mathcal{C}\left(A d_{a^{-1}}^{\star} \theta\right)\right)=a \iota_{A d_{a^{-1}} \xi} \mathcal{C}\left(A d_{a^{-1}}^{\star} \theta\right)=<A d_{a^{-1}}^{\star} \theta, A d_{a^{-1}} \xi>=<\theta, \xi>
$$

Ora, mas repare que $C$ ser invariante equivale a dizer que $\mathcal{C}$ é $G$ equivariante, isto é, para todo $a \in G, \mathcal{C}=a \circ \mathcal{C} \circ A d_{a^{-1}}^{\star}$. Então, fazendo a média sobre $\mathcal{C}$

$$
\int_{G} a \circ \mathcal{C} \circ A d_{a^{-1}}^{\star} d a
$$

nos dá um novo $\mathcal{C}$ que é $G$-equivariante. Ou seja, provamos o seguinte:

Lema 5.3.3 Se $G$ é compacto, uma $G^{*}$-álgebra é do tipo $(\boldsymbol{C})$ se, e somente se, existir elementos que satisfazem 5.3.1.

\section{4}

\section{O Subcomplexo Básico e a Cohomologia Equivariante de $G^{*}$-álgebras}

Lembre que se $G$ é compacto e age livremente sobre $M$, então o mapa

$$
\pi: M \rightarrow M / G
$$

é um $G$ fibrado principal. Defina $X=: M / G$. Temos então que

$$
\pi^{*} \Omega(X) \subset \Omega(M)
$$


é um subcomplexo. Repare que $\pi^{*} \Omega(X)$ é isomorfo a $\Omega(X)$, já que, $\pi^{*}$ é claramente injetivo. Chamaremos este subcomplexo de complexo de formas básicas, já que, são imagens de formas na base $X$ pelo mapa $\pi^{*}$.

Como os elementos de cada classe em $X$ são dados pela órbita de $G$, temos que se $\omega \in \pi^{*} \Omega(X)$ então $\omega$ é $G$-invariante. Além disso $\omega$ satisfaz 5.3.2 já que $\iota_{a}$ age na órbita $T M$ por $G$, mas como $\omega$ é uma forma básica, ela é invariante na órbita de $G$. Assim, como consequência, se $G$ é conexo, para $a=1, \ldots, n$, temos que

$$
\mathcal{L}_{a} \omega=0
$$

Como de praxe, iremos generalizar o conceito de forma básica para $G^{*}$-módulos. Dado um $G^{*}$-módulo $A$, definiremos $A_{\text {bas }}$ como o conjunto dos elementos de $A$ que são $G$-invariantes e satisfazem a condição 5.3.2. Novamente, se $G$ é conexo a condição de $G$-invariância pela equação 5.4.1. Os elementos de $A_{b a s}$ são chamados básicos. Agora, repara que se $\omega \in A_{b a s}$, então $\iota_{a} d \omega=\mathcal{L}_{a} \omega \in A_{\text {bas }}$ por 5.0.2. Logo, $d A_{b a s} \subset A_{b a s}$.

Assim, $A_{b a s}$ é um subcomplexo de $A$ nos permitindo definir a cohomologia de $A_{b a s}$ induzida pela cohomologia de $A$. A denotemos por $H_{b a s}(A)$ em vez do extensivo $H\left(A_{b a s}, d\right)$.

Repare que a única ação relevante que precisamos estudar em $A_{b a s}$ é $d$, já que, por definição $\rho(a), \mathcal{L}_{\xi}$ e $\iota_{\xi}$ agem trivialmente em $A_{b a s}$. Além disso, caso $A$ seja uma $G^{*}$-álgebra, como $G$ atua por automorfismo e $g_{-1}$ como derivação, temos que $A_{b a s}$ é uma $G^{*}$-subálgebra. E como já vimos, $H_{b a s}(A)$ herda uma estrutura de álgebra.

O que falta na estrutura de subcomplexos básico é que o mapa entre $G^{*}$-módulos induza em mapas na cohomologia básica. Mas isto é imediato. Se $\phi: A \rightarrow B$ é um $G^{*}$-módulo, pelas equações 5.1.5, 5.1.6, 5.1.7 e 5.1.8 temos que

$$
\phi\left(A_{b a s}\right) \subset B_{b a s} .
$$

Assim, a restrição de $\phi$ às formas básicas induz um mapa linear na cohomologia, dado por,

$$
\phi_{b}: H_{b a s}(A) \rightarrow H_{b a s}(B)
$$

E, é claro, se $\phi$ é um homomorfismo de $G^{*}$-álgebras, $\phi_{b}$ é um homomorfismo de álgebras. 
Agora, estabelecido o que é o subcomplexo básico e a cohomologia básica de uma $G^{*}$-álgebra (ou $G^{*}$-módulo) que satisfaz a condição $(\mathbf{C})$, estamos preparados para definir a cohomologia equivariante de uma $G^{*}$-álgebra (ou $G^{*}$-módulo) ordinário. E como podemos ver no apendice $\mathrm{B}$, esta nova cohomologia é equivalente, no caso da variedade ser compacta, a cohomologia equivariante topológica. Em outras palavras, a cohomologia equivariante de $M$, caso seja compacta, sobre a ação livre de $G$, é a mesma que cohomologia equivariante de $\Omega(M)$ como $G^{*}$-módulo.

Para definir a cohomologia equivariante, lembremos que dado $B$ uma $G^{*}$-álgebra que satisfaz a condição $(\mathbf{C})$, temos que $A \otimes B$ é uma $G^{*}$-álgebra que satisfaz a condição $(\mathbf{C})$ caso $A$ seja uma $G^{*}$-álgebra. Desta forma, definimos:

Definição 5.4.1 Seja E uma $G^{*}$-álgebra acíclica que satisfaz a condição $(\boldsymbol{C})$. Definimos a cohomologia equivariate de uma $G^{*}$-álgebra ordinária $A$ como:

$$
H_{G}(A):=H_{b a s}(A \otimes E)=H\left((A \otimes E)_{b a s}, d\right)
$$

A definição acima é análoga, só esquecendo a estrutura de álgebra, para $G^{*}$-módulos.

Vale ressaltar que estamos generalizando uma situação que vimos do ponto de vista topológico onde consideramos $G$ compacto. Desta forma, também estamos assumindo nessa definição que $G$ é compacto.

A definição da cohomologia equivariante de $G^{*}$-álgebras é semelhante a definição de cohomologia equivariante topológica, além de carregar os mesmos possíveis problemas: A definição vale para qualquer $E G^{*}$-álgebra acíclica? Existe um tal $E$ que satisfaça as condições?

Ambas as perguntas serão respondidas de forma positiva, enquanto que a primeira questão será respondida posteriormente (onde a hipótese de $G$ compacto é necessária), a segunda já será vista no próximo capítulo com a álgebra de Weil que, como iremos ver, é a mais simples $G^{*}$-álgebra acíclica que satisfaz a condição $(\mathbf{C})$ e nos proporcionará resultados interessantes que nos permitirá, no fim deste trabalho, chegar em uma fórmula para calcular a cohomologia do fibrado flag homogêneo. 
Supondo inicialmente que a definição 5.4 .1 é boa no sentido de estar bem definida, considere $\phi: A \rightarrow B$ um morfismo de $G^{*}$-módulos. Podemos considerar o mesmo $E$ para calcular a cohomologia equivariante de ambos, $A$ e B. Assim, o mapa

$$
\phi \otimes I d: A \otimes E \rightarrow B \otimes E
$$

é um morfismo de $G^{*}$-módulos. Isto nos permite definir o mapa

$$
\phi_{G}: H_{G}(A) \rightarrow H_{G}(B)
$$

onde $\phi_{G}:=(\phi \otimes I d)_{b}$. E como a validade da definição 5.4.1 garante a independência da escolha de $E$, segue que

$$
A \mapsto H_{G}(A), \phi \mapsto \phi_{G}
$$

é um funtor.

Lema 5.4.2 Se dois $G^{*}$-morfismos, $\phi_{0}: A \rightarrow B$ e $\phi_{1}: A \rightarrow B$, são homotópicos em cadeia, então

$$
\left(\phi_{0}\right)_{G}=\left(\phi_{1}\right)_{G}
$$

Prova: Como $\phi_{0}$ e $\phi_{1}$ são homotópicos em cadeia, segue que os mapas induzidos na cohomologia são iguais, isto é, $\left(\phi_{0}\right)_{*}=\left(\phi_{1}\right)_{*}$. Além disso, temos que

$$
\left(\phi_{1}\right)_{G}-\left(\phi_{0}\right)_{G}=\left(\phi_{1} \otimes I d\right)_{b}-\left(\phi_{0} \otimes I d\right)_{b}=\left(\left(\phi_{1}-\phi_{0}\right) \otimes I d\right)_{b}
$$

Logo, como o mapa $\left(\left(\phi_{1}-\phi_{0}\right) \otimes I d\right)_{b}$ é induzido por $\left(\phi_{1}-\phi_{0}\right)_{*} \otimes I d=$ $\left(\left(\phi_{1}\right)_{*}-\left(\phi_{0}\right)_{*}\right) \otimes I d$ já que $\phi_{0}$ e $\phi_{1}$ leva elementos básicos em elementos básicos, segue que $\left(\phi_{1}\right)_{G}-\left(\phi_{0}\right)_{G}=0$.

Neste momento, convido o leitor, caso tenha interesse, a olhar no apêndice B o teorema de de Rham, ele mostrará que, caso $G$ e $M$ sejam compactos, a cohomologia equivariate no caso topológico e a cohomologia equivarinte no caso de $G^{*}$-módulos são equivalentes. 


\section{6 \\ Álgebra de Weil}

Neste capítulo iremos provar a existência de uma $G^{*}$-álgebra acíclica que satisfaz a condição $(\mathbf{C})$, o que é necessário para dar sentido a definição 5.4.1. Porém, mais do que provar, iremos exibir uma $G^{*}$-álgebra que satisfaz estas condições. Esta será a chamada álgebra de Weil. Um ponto interessante em relação a ela, que ficará claro mais para frente, é que ela é uma $G^{*}$-álgebra cuja algumas estruturas já são induzidas de uma perspectiva geométrica. Desta forma a sua construção se torna mais simples do que uma $G^{*}$-álgebra ordinária.

Boa parte da construção deste capítulo é uma exposição da teoria de Chern-Weil com base na versão de Weil.

A álgebra de Weil vai ser muito importante para este trabalho, sendo utilizada em vários resultados, inclusive o resultado que é foco desta dissertação. Comecemos então com algumas construções que precedem a álgebra de Weil.

Seja $V$ um espaço vetorial de dimensão $n$. Como já vimos, $S:=S(V)$ é uma álgebra simétrica. Vale ressaltar que a consideramos como uma álgebra onde seus elementos são pares, desta forma, atribuimos a um elemento em $S^{k}=S^{k}(V)$ o grau $2 k$. Consideremos agora a álgebra exterior de $V$ dada por $\wedge(V)$, podemos considerá-la como uma superálgebra comutativa, já que temos a propriedade $x_{1} \wedge x_{2}=-x_{2} \wedge x_{1}$, com $x_{1}, x_{2} \in V$ e, portanto, $\left[x_{1}, x_{2}\right]=x_{1} \wedge x_{2}-(-1)^{1} x_{2} \wedge x_{1}=x_{1} \wedge x_{2}+x_{2} \wedge x_{1}=0$. Como para todo o elemento em $\wedge(V)$ o resultado segue, não só para os elementos em $\wedge^{1}(V)$, temos uma super álgebra comutativa. Neste caso, dizemos que um elemento em $\wedge^{k}:=\wedge^{k}(V)$ tem grau $k$.

Vale ressaltar que, quando não houver dúvidas, assim como no caso de formas, iremos esquecer o símbolo $\wedge$ e escrever o produto em $\wedge(V)$ com a notação de produto usual.

Definição 6.0.1 A álgebra de Koszul é o produto tensorial $\wedge \otimes S$. 
Vale a pena comentar neste momento o porquê de dobrarmos a dimensão de $S(V)$, ou pelo menos evidenciar o que isto acarreta. Em um primeiro momento, dobrar a dimensão dos elementos de $S(V)$ nos permite dizer que $S(V)$ também é uma super álgebra comutativa. Além disso, quando trabalhamos na álgebra de Koszul, isto nos permite desconsiderar o sinal do produto, isto é, como já vimos, temos que o produto é definido como:

$$
\left(a_{1} \otimes b_{1}\right) \cdot\left(a_{2} \otimes b_{2}\right)=(-1)^{i j} a_{1} a_{2} \otimes b_{1} b_{2}
$$

onde $i$ é o grau de $a_{2}$ e $j$ o grau de $b_{1}$. Como no nosso caso, o grau de $b_{1}$ é sempre par esquecemos o sinal. Outro ponto que ficará evidente mais tarde, e por isto não entraremos muito no assunto agora, é que esta dimensão nos permitirá ter a dimensão de $C_{G}(A)$ como o modelo de Cartan igual a da bigraduação de $C_{G}(A)$. Em um primeiro momento vale o comentário, mas ficará mais claro nos próximos capítulos junto com a definição precisa de $C_{G}(A)$.

Voltando a álgebra de Koszul, repare que os elementos $x \otimes 1 \in \wedge^{1} \otimes S^{0} \mathrm{e}$ $1 \otimes x \in \wedge^{0} \otimes S^{1}$ geram todo o $\wedge \otimes S$. E, como já comentamos, para construir uma derivação basta defini-la em seus geradores. Desta forma, definiremos o operador de Koszul, $d_{k}$, em geradores por:

$$
d_{k}(x \otimes 1)=1 \otimes x, \quad d_{k}(1 \otimes x)=0 .
$$

Repare que claramente $d_{k}^{2}=0$ nos geradores e, portanto, em todo $\wedge \otimes S$. Mostremos agora que $d_{k}$ é um operador acíclico, isto é, a única cohomologia de $d_{k}$ encontra-se em $\wedge^{0} \otimes S^{0}$.

Seja $Q$ uma outra derivação em $\wedge \otimes S$ dado nos geradores por

$$
Q(x \otimes 1)=0, \quad Q(1 \otimes x)=x \otimes 1
$$

Novamente temos que $Q^{2}=0$. Agora considere o novo operador $\left[Q, d_{k}\right]=$ $Q \circ d_{k}-d_{k} \circ Q: \wedge \otimes S \times \wedge \otimes S \rightarrow \wedge \otimes S$. Repare que $\left[Q, d_{k}\right]=I d$ nos geradores. Seja $x_{1} \cdots x_{i} \otimes 1 \in \wedge^{i} \otimes S^{1}$. Então,

$$
\begin{gathered}
{\left[Q, d_{k}\right]\left(x_{1} \cdots x_{i} \otimes 1\right)=Q d_{k}\left(x_{1} \cdots x_{i} \otimes 1\right)=} \\
=Q\left[\left(d_{k} x_{1} \otimes 1\right) x_{2} \cdots x_{i} \otimes 1-x_{1}\left(d_{k} x_{2} \cdots x_{i} \otimes 1\right)\right]= \\
=Q\left(\sum_{l=1}^{i}(-1)^{l+1}\left(x_{1} \cdots x_{l-1} \otimes 1\right)\left(d_{k} x_{l} \otimes 1\right)\left(x_{l+1} \cdots x_{i} \otimes 1\right)\right),
\end{gathered}
$$


onde representamos $x_{0}=0=x_{i+1}$. Ora, mas novamente temos que $Q\left(x_{u} \cdots x_{v} \otimes 1\right)=0$ e $Q d_{k}\left(x_{v} \otimes 1\right)=x_{v} \otimes 1$, segue então que

$$
Q\left(\sum_{l=1}^{i}(-1)^{l+1}\left(x_{1} \cdots x_{l-1} \otimes 1\right)\left(d_{k} x_{l} \otimes 1\right)\left(x_{l+1} \cdots x_{i} \otimes 1\right)\right)=i\left(x_{1} \cdots x_{i} \otimes 1\right) .
$$

De forma análoga, temos que, dado $1 \otimes y_{1} \cdots y_{j} \in \wedge^{1} \otimes S^{j}$,

$$
\left[Q, d_{k}\right]\left(1 \otimes y_{1} \cdots y_{j}\right)=j\left(1 \otimes y_{1} \cdots y_{j}\right)
$$

Assim, como $x_{1} \cdots x_{i} \otimes y_{1} \cdots y_{j}=\left(x_{1} \cdots x_{i} \otimes 1\right) \cdot\left(1 \otimes y_{1} \cdots y_{j}\right)$, segue que

$$
\left[Q, d_{k}\right]=(i+j) I d \quad \text { em } \quad \wedge^{i} \otimes S^{j}
$$

Agora, seja $z \in \wedge^{i} \otimes S^{j}$ tal que $d_{k} z=0$. Ora, mas $Q d_{k} z-d_{k} Q z=(i+j) z$. Portanto

$$
d_{k} Q z=-(i+j) z
$$

Ou seja, se $(i+j) \neq 0$ temos que

$$
z=d_{k}\left(-\frac{Q z}{(i+j)}\right)
$$

e $z$ está na imagem de $\wedge \otimes S$ por $d_{k}$. Logo $d_{k}$ é um operador acíclico.

Então, antes de introduzir a álgebra de Weil, para facilitar nossa notação, dado $x_{1}, \cdots, x_{n}$ uma base de $V$, definiremos:

$$
\theta^{i}:=x_{i} \otimes 1, \quad z_{i}:=1 \otimes x_{i}
$$

$\mathrm{e}$

$$
d_{k}:=d
$$

Desta forma temos que $d \theta^{i}=z_{i}$ e $d z_{i}=0$.

\section{1}

\section{Álgebra de Weil}

Definição 6.1.1 A álgebra de Weil é uma álgebra de Koszul cujo o espaço vetorial é $g^{*}$. Isto é,

$$
W:=\wedge\left(g^{*}\right) \otimes S\left(g^{*}\right) .
$$

Como falamos antes, a álgebra de Weil vai nos servir de exemplo de uma $G^{*}$-álgebra acíclica. Além disso, como veremos no final desta seção, ela terá a 
propriedade de que seu anel de cohomologia básica será $S\left(g^{*}\right)^{G}$.

Fica claro então que $W$ é uma álgebra acíclica em relação ao operador $d$ pelo que fizemos anteriormente. Porém, nada garante que ela é uma $G^{*}$-álgebra acíclica que satisfaz a condição $(\mathbf{C})$. Então mostraremos isso agora.

Consideremos $\xi_{1}, \cdots, \xi_{n}$ uma base de $g$. Isto nos dá origem a uma base de $g^{*}, x_{1}, \cdots, x_{n}$ e, portanto, os geradores $\theta^{1}, \cdots, \theta^{n}, z_{1}, \cdots, z_{n}$ de $W$.

Como já vimos antes, $G$ age em $g^{*}$ através da representação coadjunta $A d^{\star}$ que atua como um automorfismo de super álgebra em $W$. Porém, outra forma de ver é pensando inicialmente na representação adjunta, isto é, considere $a \in G$, temos o mapa suave

$$
A d(a): g \rightarrow g
$$

Este mapa, como já vimos, induz uma representação de $G$ em $\Omega(g)$ dado por

$$
\left(A d^{-1}(a)\right)^{*} \omega
$$

onde $\omega \in \Omega(g)$. Ora, mas $g^{*}$ está contido em $\Omega^{1}(g)$, assim, como $\left(A d^{-1}\right)^{*}=A d^{\star}$, o operador

$$
\mathcal{L}_{\xi}:=\left.\frac{d}{d t} A d^{\star}(\exp t \xi)\right|_{t=o}
$$

está bem definido operando em $g^{*}$. E é claro, definindo um novo operador, que também denotaremos por $\mathcal{L}_{\xi}$, como $\mathcal{L}_{\xi} \otimes 1+1 \otimes \mathcal{L}_{\xi}$ em $W$, temos que a equação 5.1.1 é satisfeita. Esta construção pode ficar mais evidente ao vermos o que acontece nos geradores (o que faremos a seguir) e depois estendermos como uma derivação de grau 0.

Ainda sobre $\mathcal{L}_{\xi}$ podemos fazer mais considerações. Consideremos uma outra representação adjunta, mas agora de $g \rightarrow g l(g)$, definida por

$$
\operatorname{ad}(\xi)(\nu)=[\xi, \nu]
$$

Repare então que $A d(\exp t \xi)(\nu)=d\left(r_{\exp -t \xi}\right)_{\exp t \xi}\left(d\left(L_{\exp t \xi}\right)_{e}(\nu)\right)$. Assim, como $\nu$ é invariante por translação, temos que $d\left(L_{\exp t \xi}\right)_{e}(\nu)=\nu(\exp t \xi)$. Assim,

$$
A d(\exp t \xi)(\nu)=d\left(\xi_{-t}\right)_{\xi(e)}\left(\nu\left(\xi_{t}(e)\right)\right)
$$


Logo, derivando, temos que

$$
\frac{d}{d t}\left(\left.A d(\exp t \xi)(\nu)\right|_{t=0}=[\xi, \nu](1)\right.
$$

Usando isto, não é difícil ver que $\mathcal{L}_{\xi_{i}}\left(x_{j}\right)=a d^{\star}\left(x_{i}\right)\left(x_{j}\right)=$ $\left(-\operatorname{ad}\left(\xi_{i}\right)\left(\xi_{j}\right)\right)^{*}=-c_{i k}^{j} x_{k}$. Portanto, segue que

$$
\mathcal{L}_{a} \theta^{b}=-c_{a k}^{b} \theta^{k}
$$

e

$$
\mathcal{L}_{a} z_{b}=-c_{a k}^{b} z_{k}
$$

Além disso, o operador $d$ claramente comuta com $A d^{\star}$, desta forma é $G$-equivariante e satisfaz a equação 5.1.4. Quanto a veracidade da equação 5.1.2 é consequência do caso geométrico, isto é, a definição de $\mathcal{L}_{\xi}$ que fizemos vem do caso geométrico e sabemos que a equação 5.1.2 é válida nesse caso.

Nos falta agora verificar a equação 5.1.3, ou melhor, nos falta definir o que será o operador $\iota_{\xi}$. O que iremos fazer é justamente criar este operador para satisfazer o que queremos, isto é, iremos criar o operador $\iota_{\xi}$ de forma a termos uma $G^{*}$-ação que é acíclica e possui a propriedade (C) com os $\theta^{i}$ sendo os elementos de conexão. Vale lembrar que se $G$ é conexo, como estaremos supondo, a propriedade (C) significa dizer que além da equação 6.1.2, também temos que contemplar a equação 5.3.1. Assim, primeiro definimos a ação nos elementos de conexão, isto é,

$$
\iota_{a} \theta^{b}=\delta_{a}^{b}
$$

Como além de satisfazer as equações 5.1.1, 5.1.2, 5.1.3 e 5.1.4, a ação de $\bar{g}$ tem que ser como super derivação em $W$, temos que satisfazer a igualdade $d \iota_{a}+\iota_{a} d=\mathcal{L}_{a}$, e portanto

$$
\iota_{a} z_{b}=\iota_{a} d \theta^{b}=\left(\iota_{a} d+d \iota_{a}\right) \theta^{b}=\mathcal{L}_{a} \theta^{b} .
$$

Logo,

$$
\iota_{a} z_{b}=-c_{a k}^{b} \theta^{k}
$$

Assim, definimos a ação de $\iota_{a}$ estendendo como derivação de grau -1 para todo $W$. Para verificar que a equação 5.1 .3 basta observar que assim como fizemos no capítulo 4 , isto é consequência de $\left[d, \iota_{a}\right]=\mathcal{L}_{a}$ e da equação 5.1.2. Logo a equção 5.1 .3 vale neste caso.

Resta, então, mostrar que $\bar{g}$ atua como uma super derivação e é claro, para isto basta verificar nos geradores. Primeiro, como já usamos para de- 
finir o operador $\iota_{a}$, é imediato que $\left[d, \iota_{a}\right]=\mathcal{L}_{a}$. Além disso, claramente $\left[\iota_{a}, \iota_{b}\right]\left(\theta^{j}\right)=0$. Teríamos que verificar então que $\left[\iota_{a}, \iota_{b}\right]\left(z_{j}\right)=0$. Ora, mas $\left[\iota_{a}, \iota_{b}\right]\left(z_{j}\right)=-c_{a k}^{j} \iota_{b} \theta^{k}+c_{b k}^{j} \iota_{a} \theta^{k}=0$, pois $\iota_{l} \theta^{k}$ é igual a 1 caso $k=l$ e 0 caso contrário.

Nos resta então provar que $\left[\mathcal{L}_{a}, \iota_{b}\right]=c_{a b}^{k} \iota_{k}$ e $\left[\mathcal{L}_{a}, \mathcal{L}_{b}\right]=c_{a b}^{k} \mathcal{L}_{k}$. Ora, mas

$$
\left[\mathcal{L}_{a}, \mathcal{L}_{b}\right]=\left[\mathcal{L}_{a},\left[d, \iota_{b}\right]\right]=\left[d,\left[\mathcal{L}_{a}, \iota_{b}\right]\right]=c_{a b}^{k}\left[d, \iota_{k}\right]=c_{a b} \mathcal{L}_{k}
$$

caso $\left[\mathcal{L}_{a}, \iota_{b}\right]=c_{a b}^{k} \iota_{k}$, nos restando assim provar este resultado. Entretanto,

$$
\begin{gathered}
{\left[\mathcal{L}_{a}, \iota_{b}\right]\left(\theta^{i}\right)=\iota_{b} c_{a k}^{i} \theta^{k}} \\
{\left[\mathcal{L}_{a}, \iota_{b}\right]\left(z_{i}\right)=\mathcal{L}_{a} \mathcal{L}_{b} \theta^{i}+c_{a k}^{i} \iota_{b} z_{k}=\mathcal{L}_{a} \mathcal{L}_{b} \theta^{i}+c_{a k}^{i} \mathcal{L}_{b} \theta^{k}=c_{a b}^{k} \mathcal{L}_{k} \theta^{i}=c_{a b}^{k} \iota_{k} \theta^{i} .}
\end{gathered}
$$

$\operatorname{Logo}\left[\mathcal{L}_{a}, \iota_{b}\right]=c_{a b}^{k} \iota_{k}$. Desta forma provamos:

Teorema 6.1.2 W é uma $G^{*}$-álgebra acíclica que satisfaz a condição (C).

Nosso objetivo agora é associar a parte horizontal de $W \operatorname{com} S\left(g^{*}\right)$ afim de dar outras interpretações para $W$, o que nos permitirá ter mais desenvolvimentos algébricos. Neste sentido provemos o seguinte:

\section{Lema 6.1.3}

$$
W=\wedge\left(g^{*}\right) \otimes W_{h o r}
$$

Prova: Defina

$$
\mu^{b}:=z_{b}+\frac{1}{2} c_{j k}^{b} \theta^{j} \theta^{k}
$$

Mostremos que $\mu^{b}$ é horizontal.

$$
\iota_{a} \mu^{b}=\iota_{a} z_{b}+\left(\frac{1}{2} c_{j k}^{b}\right)\left(\delta_{a}^{j}-\theta^{j} \delta_{a}^{k}\right)=\iota_{a} z_{b}+c_{a l}^{b} \theta^{l}=0 .
$$

Logo os elementos $\mu^{b}$ são horizontais em $W$. E é claro que $z_{b}=\mu^{b}-\frac{1}{2} c_{j k}^{b} \theta^{j} \theta^{k}$ e, portanto, $\theta^{a}$ e $\mu^{b}$ são geradores de $W$. Ou seja, podemos ver $W$ através do produto tensorial da álgebra exterior em $\theta$ com o anel polinômial $\mathbb{C}\left[\mu^{1}, \cdots, \mu^{n}\right]$. E ainda, fica claro que um elemento é horizontal em $W$ se e somente se ele está em $\mathbb{C}\left[\mu^{1}, \cdots, \mu^{n}\right]$.

Logo $W=\wedge\left(g^{*}\right) \otimes W_{\text {hor }}$

Desta forma conseguimos a relação que queríamos entre a parte horizontal de $W$ e $S\left(g^{*}\right)$. Se identificarmos $\mathbb{C}\left[\mu^{1}, \cdots, \mu^{n}\right]$ com $S\left(g^{*}\right)$, temos a igualdade 


$$
W_{\text {bas }}=S\left(g^{*}\right)^{G}=\left\{p \in S\left(g^{*}\right) \mid a \cdot p=p \forall a \in G\right\}
$$

onde $W_{\text {bas }}$ são os elementos $G$-invariantes de $W_{\text {hor }}$ e $a \cdot p$ representa a ação de $G$ em $S\left(g^{*}\right)$.

Precisamos agora entender como funciona os operdadores $\mathcal{L}_{a}$ e $d$ em $\mu^{b}$. Repare que $\mu$ é obtido ao adicionar $\frac{1}{2} c_{j k}^{b} \theta^{j} \theta^{k}$ a $z_{b}$. Ora, mas sabemos que dado um mapa bilinear de $g \times g$ em $g$, existe um único levantamento linear de $g \otimes g$ em $g$, isto é,

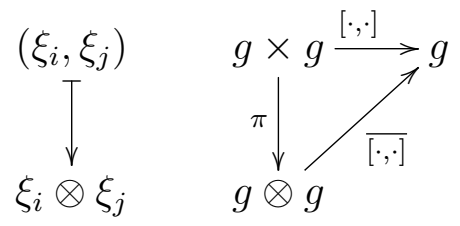

Mas,

$$
\overline{[\cdot, \cdot]} \in H^{\circ o m}{ }_{\mathbb{C}}(g \otimes g, g) \cong(g \otimes g)^{*} \otimes g \cong g^{*} \otimes g^{*} \otimes g
$$

e

$$
\overline{[\cdot, \cdot]}\left(\xi_{i}, \xi_{j}\right)=c_{i j}^{k} \xi_{k},
$$

portanto podemos ver o elemento $c_{i j}^{k} \xi_{k} \theta^{i} \otimes \theta^{j}$ como o mapa $g \otimes g \rightarrow g$ a partir do colchete de Lie e do elemeto $c_{i j}^{k} \theta^{i} \theta^{j}$.

Portanto, com um pouco de trabalho podemos ver que

$$
\mathcal{L}_{a}\left(\frac{1}{2} c_{i j}^{b} \theta^{i} \theta^{j}\right)=-c_{a k}^{b}\left(\frac{1}{2} c_{i j}^{k} \theta^{i} \theta^{j}\right)
$$

e, assim,

$$
\mathcal{L}_{a} \mu^{b}=-c_{a k}^{b} \mu^{k}
$$

Agora, sabemos que $d \theta^{a}=z_{a}$, portanto

$$
d \theta^{a}=-\frac{1}{2} c_{i j}^{a} \theta^{i} \theta^{j}+\mu^{a} .
$$

Esta equação é conhecida como equação estrutural de Cartan. Logo

$$
d \mu^{a}=\left(\frac{1}{2} c_{i j}^{a}\right)\left(d \theta^{i} \theta^{j}-\theta^{i} d \theta^{j}\right),
$$

então, como podemos ver em [GS],

$$
d \mu^{a}=-c_{i j}^{a} \theta^{i} \mu^{j} .
$$

Equação conhecida como identidade de Bianchi. 
Assim, usando as equações anteriores, também podemos escrever

$$
d \mu^{a}=\left(\theta^{b} \mathcal{L}_{a}\right) \mu^{a}
$$

Com isto podemos provar o seguinte:

Teorema 6.1.4 O anel de cohomologia básico de $W$ é $S\left(g^{*}\right)^{G}$.

Prova: De fato, podemos ver $\theta^{b} \mathcal{L}_{b}$ como uma derivação pois é uma derivação seguida de produto, além de ter grau 1 pois $d$ tem grau 1 .

Como $\mu^{a}$ gera $W_{\text {hor }}$, temos que para todo $\omega \in W_{\text {hor }}$

$$
d \omega=\theta^{b} \mathcal{L}_{b} \omega
$$

Logo, como por definição $\mathcal{L}_{a} \omega=0$ para todo $\omega \in W_{b a s}$, segue que $d \omega=0$ para todo $\omega \in W_{\text {bas }}$.

$$
\text { Como } W_{\text {bas }}=S\left(g^{*}\right)^{G} \text { temos o resultado. }
$$

\section{2}

\section{$W$ a $G^{*}$-álgebra Mais Simples e o Mapa de Chern-Weil}

A ideia desta seção inicialmente é entender, dentro de um certo ponto de vista, $W$ dentro de qualquer $G^{*}$-álgebra de forma que isto se mantenha na cohomologia básica. Isto nos permitirá depois entendermos $W$ como a $G^{*}$ álgebra mais simples que satisfaz a condição (C). A construção que faremos é, de certa forma, um análogo algébrico do teorema 3.2.4 que é puramente geométrico, onde se considerarmos $M$ e $E$ variedades diferenciáveis em que $G$ age livremente, e $E$ contrátil, cada seção transversal $s: M / G \rightarrow(M \times E) / G$ determina um único mapa $G$-equivariante, a menos de homotopia, $\epsilon: M \rightarrow E$ que faz o diagrama

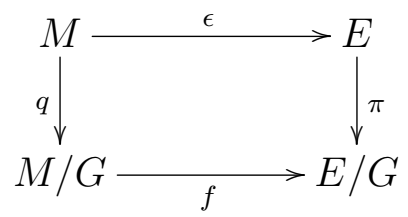

comutar.

Teorema 6.2.1 Seja $A$ uma $G^{*}$-álgebra do tipo $(\boldsymbol{C})$. Existe um único homomorfismo de $G^{*}$-álgebra a menos de homotopia em cadeia $\rho: W \rightarrow A$. 
Prova:

Aqui voltaremos a suposição de que $G$ é conexo como em geral faremos. Repare que a construção da álgebra de Weil neste caso fica um pouco mais fácil, ja que as equações 5.1.2, 5.1.3, 5.1.4 serão consequência da equação 5.1.1.

Como $A$ é uma $G^{*}$-álgebra que satisfaz a condição $(\mathbf{C})$, existem $\theta_{A}^{a} \in A_{1}$ que satisfazem as equações 5.3.3 e 5.3.1. Logo, definindo

$$
\rho\left(\theta^{a}\right)=\theta_{A}^{a}
$$

o mapa $\rho$ se estende unicamente para todo $W$ uma vez que ele é gerado livremente por $\theta^{a}$ e $d \theta^{a}$, pois $\rho$ e $d$ comutam. Desta forma, existe tal $\rho$.

Agora, caso $\rho_{0}$ e $\rho_{1}$ satisfaçam as hipóteses, defina $\rho_{t}: W_{1} \rightarrow A$, onde $W_{1}$ é o espaço vetorial gerado unicamente pela combinação dos $\theta^{a}$ e

$$
\rho_{t}=(1-t) \rho_{0}+t \rho_{1}
$$

O mapa está bem definido pois $A$ é um espaço vetorial. Além disso repare que, por hipótese, $\rho_{0}$ e $\rho_{1}$ são $G$-equivariantes e comutam com $\iota_{\xi}$, portanto o mesmo acontece com $\rho_{t}$. Assim podemos estender $\rho_{t}$ para um homomorfismo de $G^{*}$-álgebra.

Precisamos agora definir o mapa de homotopia em cadeia. Defina então $Q_{t}$ o mapa definido nos geradores por

$$
Q_{t}\left(\theta^{a}\right)=0 \quad \text { e } \quad Q_{t}\left(d \theta^{a}\right)=\frac{d}{d t} \rho_{t}\left(\theta^{a}\right)=-\rho_{0}\left(\theta^{a}\right)+\rho_{1}\left(\theta^{1}\right)
$$

Depois estendemos $Q_{t}$ para todo $W$ pela equação 5.2.6 onde $\rho_{t}$ faz o papel de $\phi$. Isto, como vimos, garante que $Q_{t}$ é uma homotopia em cadeia relativa a $\rho_{t}$. Assim, pela equação 5.2.5,

$$
Q:=\int_{0}^{1} Q_{t} d t
$$

é uma homotopia em cadeia entre $\rho_{0}$ e $\rho_{1}$.

Podemos então definir $\mu_{A}^{a}$ em $A$ por $\mu_{A}^{a}=: \rho\left(\mu^{a}\right)$. Logo as equações de Cartan e identidade de Bianchi, também conhecidas como equações estruturais 
de Cartan, são satisfeitas, isto é

$$
\begin{gathered}
d \theta_{A}^{a}=-\frac{1}{2} c_{i j}^{a} \theta_{A}^{i} \theta_{A}^{j}+\mu_{A}^{a} \\
d \mu_{A}^{a}=-c_{i j}^{a} \theta_{A}^{i} \mu_{A}^{j} .
\end{gathered}
$$

Repare também que em particular, como $\rho$ é um $G^{*}$-morfismo e, portanto, mapeia $W_{b a s}$ em $A_{b a s}$, e $\rho$ é único a menos de homotopia em cadeia, temos como consequência o seguinte:

Teorema 6.2.2 Existe um mapa canônico

$$
\kappa_{G}: S\left(g^{*}\right)^{G} \rightarrow H_{b a s}(A)
$$

Este é o poderoso mapa de Chern-Weil ou simplesmente, como também é conhecido, homomorfismo característico.

No próximo capítulo iremos dissertar especificamente sobre a comutação de uma ação. Porém, vale comentar agora um exemplo das notas de [GS] que dialoga com o que estamos vendo e com o que veremos.

Exemplo 6.2.3 Sejam $G$ e $K$ grupos de Lie que agem $M$. Suponha que G seja compacto e que age livemente em $M$. Consideremos então $M$ uma $(G \times K)$ variedade. Um $G^{*}$-módulo interessante é o complexo equivarinate de de Rham $\Omega_{K}(M)$ (como temos uma ação de $G \times K$ em $M$, podemos ver $\Omega_{K}(M)$ como um $G^{*}$-módulo ou $\Omega_{G}(M)$ como um $K^{*}$-módulo). Com a teoria que estudamos até aqui e que veremos no próximo capítulo, podemos ver o mapa Chern Weil em uma versão diferente, neste caso como

$$
\kappa_{G}: S\left(g^{*}\right)^{G} \rightarrow H_{K}^{*}(M / G) .
$$

Este mapa tem como imagem o anel das classes características equivariantes de $M / G$.

O exemplo acima é complicado de compriender e necessitamos um pouco mais de teoria. Convido o leitor a tentar entender um pouco mais o exemplo após ver 7.5. O exemplo fica mais claro ao ver que $\Omega_{{b a s_{G^{*}}}}(M) \cong \Omega(M / G)$ e que o mapa $\kappa_{G}$ acima é o mesmo que veremos em 7.5. 


\section{3}

\section{$W^{*}$-módulos}

O mapa $\rho$ que trabalhamos na seção anterior nos permite pensar em qualquer $G^{*}$-álgebra que satisfaz a condição $(\mathbf{C})$ como uma álgebra sobre $W$, ou, em particular, um módulo sobre $W$. Para generalizar esta noção definimos:

Definição 6.3.1 Um $W^{*}$-módulo é um $G^{*}$-módulo $B$ que também é um módulo sobre $W$ de tal forma que o mapa

$$
\begin{aligned}
& W \otimes B \rightarrow B \\
& w \otimes b \mapsto w b
\end{aligned}
$$

é um morfismo de $G^{*}$-módulos. Uma $W^{*}$-álgebra é uma $G^{*}$-álgebra que também é um $W^{*}$-módulo.

Em geral, não exibiremos o morfismo correspondente, deixando assim implícito que $w b$ representa a ação de $w \in W$ em $b \in B$.

Não é difícil ver que uma $G^{*}$-álgebra que satisfaz a condição $(\mathbf{C})$ é uma $W^{*}$-álgebra.

O resultado que queremos mostrar agora é que todo elemento em um $W^{*}$-módulo pode ser escrito como uma soma exclusiva de produtos de formas de conexão por formas horizontais. Escrevamos isto da seguinte forma:

Considere o multi-índice $I:=\left(i_{1}, \cdots, i_{r}\right)$ onde $1 \leq i_{1}<i_{2}<\cdots<i_{r} \leq$ $n$. Assim, seja $\theta^{I}=\theta^{i_{1}} \cdots \theta^{i_{r}}$ o monômio em $\theta^{a} \in W$. Desta forma $\theta^{I}$ age como um operador em $B$.

Teorema 6.3.2 Cada elemento de um $W^{*}$-módulo $B$ pode ser escrito unicamente pela soma

$$
\theta^{I} h_{I}
$$

onde $h_{I} \in B_{\text {hor }}$.

Prova: Provemos então por indução. Considere $\theta^{J} h_{J}$ onde $J=\left(j_{1}, \cdots, j_{m}\right)$, $1 \leq j_{1}<\cdots<j_{m} \leq k-1$ e $\iota_{a} h_{J}=0$ para $a=1, \cdots, k-1$.

Como o resultado é evidentemente verdadeiro para $0, k=1$ é automaticamente verdade. Agora façamos o passo indutivo, suponha que o resultado é 
verdadeiro para $k-1$, provemos que vale para $k$. Defina

$$
a_{J}:=\iota_{k} h_{J} \quad \text { e } \quad b_{J}:=h_{J}-\theta^{k} a_{J} .
$$

Por hipóteses o resultado é válido para $a_{J}$ e também para $b_{J}$.

Logo,

$$
\theta^{J} h_{J}=\theta^{J} h_{J}+\theta^{J} \theta^{k} a_{J}-\theta^{J} \theta^{k} a_{J}=\theta^{J} b_{J}+\theta^{J} \theta^{k} a_{J}
$$

e assim, o resultado é estendido indutivamente (perceba que o caso $k=j+1$ é o teorema).

Ora, mas $\wedge\left(\theta^{1}, \cdots, \theta^{n}\right)$ é isomorfo $\wedge\left(g^{*}\right) \otimes 1 \subset W$, então como consequência do teorema anterior temos que:

Corolário 6.3.3 O mapa

$$
\wedge\left(g^{*}\right) \otimes B_{h o r} \rightarrow B, \theta^{I} \otimes h \mapsto \theta^{I} h
$$

é uma bijeção. 


\section{7 \\ Modelos e o isomorfismo de Mathai-Quillen}

Neste capítulo iremos finalmente provar que a cohomologia equivariante não depende da escolha da $G^{*}$-álgebra acíclica e, assim, a definição da cohomologia equivariante estará bem definida.

O título deste capítulo já indica o que iremos fazer, vamos passar do modelo de Weil, que trabalhamos no capítulo anterior, onde representamos a cohomologia equivariante de um $G^{*}$-módulo (ou $G^{*}$-álgebra) $B$ a partir de $(B \otimes W)_{b a s}$, para o modelo de Cartan através do poderoso isomorfismo de Mathai-Quillen. Como iremos ver, esta passagem para o modelo de Cartan tornará mais fácil de se trabalhar, já que, o cálculo de $(B \otimes W)_{b a s}$ pode ser bem complicado. Porém, o operadador $d$ no novo modelo é mais complexo e precisaremos estudá-lo um pouco mais.

Começaremos então com a passagem dos modelos. A passagem não depende de $W$, só da estrutura de $W^{*}$-módulo, desta forma iremos trabalhar com um $W^{*}$-módulo qualquer $A$ em vez de $W$. Além disso, caso $B$ seja um $G^{*}$-módulo (ou $G^{*}$-álgebra), assim como em [GS], iremos trabalhar com $A \otimes B$ em vez de $B \otimes A$ por motivos técnicos. Isto irá, por exemplo, contornar problemas de sinais.

Para ficar mais claro, estaremos definindo o modelo de Weil pelo seguinte:

Definição 7.0.1 Seja B um $G^{*}$-módulo. O espaço

$$
(W \otimes B)_{b a s}
$$

junto com o operador diferencial d restrito a $(W \otimes B)_{\text {bas }}$ é o modelo de Weil para a cohomologia equivariante de $B$.

\section{1}

\section{Isomorfismo de Mathai-Quillen}

Seja $A$ um $W^{*}$-módulo e $B$ um $G^{*}$-módulo. Como falamos antes, $A$ ser um $W^{*}$-módulo significa que podemos ver os elementos de $W$ como operadores 
em $A$. Assim, considere $\xi_{1}, \ldots, \xi_{n}$ uma base de $g$. Então, como consequência temos $\theta^{1}, \ldots, \theta^{n}$, geradores de conexão, e $\mu^{1}, \ldots, \mu^{n}$, geradores de curvatura, em $W$.

Podemos então definir

$$
\gamma:=\sum_{a=1}^{n} \theta^{a} \otimes \iota_{a}=\theta^{a} \otimes \iota_{a}
$$

um endomorfismo de $A \otimes B$. Lembrando que $\operatorname{End}(A \otimes B)$ admite uma estrutura de álgebra de Lie. Podemos fazer algumas considerações sobre $\gamma$ :

1. $\gamma$ tem grau zero.

De fato, $\iota_{a}$ diminui o grau de um elemento em $B$ em 1 quanto $\theta^{a}$ aumenta o grau de um elemento $A$ em 1 .

2. A definição de $\gamma$ independe da escolha da base.

Isto se deve ao fato que escolhendo outra base, ação de $\theta^{a}$ seguirá da mesma forma pela construção que fizemos da ação de $W$ em $A$. O mesmo vale para $\iota_{a}$ seguindo de $\theta^{a}$.

3. $\gamma$ é nilpotente.

Basta perceber $\theta^{a} \in \wedge\left(g^{*}\right)$ e, por definição de $\wedge\left(g^{*}\right)$, os elementos de grau maior que a dimensão de $g^{*}$ são 0 . Logo $\gamma^{n+1}=0$

4. $\gamma$ é invariante sobre a ação coadjunta de $G$.

De fato, seja $a \in G$, temos que

$$
A d(a) \xi_{1}, \ldots, A d(a) \xi n
$$

forma uma nova base para $g$ que tem como base dual para $g^{*}$

$$
A d^{\star}\left(a^{-1}\right) \theta^{1}, \ldots, A d^{\star}\left(a^{-1}\right) \theta^{n}
$$

Mas sabemos que $<\theta^{a}, \xi_{b}>=\delta_{a b}$ e que vale a igualdade

$$
<\theta^{a}, \xi_{b}>=<A d^{\star}\left(c^{-1}\right) \theta^{a}, A d(c) \xi_{b}>.
$$

Assim, como $\gamma$ independe da escolha da base, também podemos escrever $\gamma$ da forma

$$
\gamma=A d^{\star}\left(c^{-1}\right) \theta^{a} \otimes \iota_{A d(c) \xi_{a}}
$$


Mostremos então o resultado. Consideremos $\alpha \otimes \beta \in A \otimes B$. Temos que:

$$
\begin{gathered}
\gamma(c(\alpha \otimes \beta))=\gamma(c \alpha \otimes c \beta)=\left(\theta^{a} c \alpha \otimes \iota_{a} c \beta\right)= \\
=\left(\theta^{a} c \alpha \otimes c \iota_{A d(c) \xi_{a}} \beta\right)=\left(c \cdot c^{-1} \theta^{a} c \alpha \otimes c \iota_{A d(c) \xi_{n}} \beta\right)= \\
=\left(c \cdot A d^{\star}\left(c^{-1}\right) \theta^{a} \alpha \otimes a \iota_{A d(c) \xi_{a}} \beta\right)=c\left(A d^{\star}\left(c^{-1}\right) \theta^{a} \alpha \otimes \iota_{A d(c) \xi_{a}} \beta\right)= \\
=c \gamma(\alpha \otimes \beta) .
\end{gathered}
$$

Logo, $\gamma c=c \gamma$ para todo $c \in G$.

Graças a essas considerações, o operador $\phi$ dado por

$$
\phi:=\exp \gamma=1+\gamma+\frac{1}{2 !} \gamma^{2}+\frac{1}{3 !} \gamma^{3}+\cdots
$$

está bem definido e converge, além de ser um automorfismo de $A \otimes B$ como se espera de uma expansão exponencial de um endomorfismo, assim como em [GS]. Este automorfismo leva o nome de isomorfismo de Mathai-Quillen e a partir dele que chegaremos na relação entre o modelo de Weil (definição 7.0.1) e o modelo de Cartan (definição 7.2.1).

Então, já entrando na teoria de álgebra de Lie, dado $\beta \in \operatorname{End}(A \otimes B)$ definimos, como usual,

$$
\operatorname{ad\gamma }(\beta):=[\gamma, \beta]=\gamma \beta-\beta \gamma
$$

Perceba que como $a d(\gamma)$ é nilpotente, já que, $\gamma$ o é, isto é,

$$
\left.(a d \gamma)^{2 n+1} \beta=[\gamma,[\gamma, \ldots[\gamma, \beta] \ldots]]\right]=0
$$

Desta forma $\exp (a d \gamma)$ também converge. Temos que a equação

$$
\exp (a d \gamma) \beta=\exp (\gamma) \beta \exp (\gamma)^{-1}
$$

é verdadeira. Definindo então

$$
A d(\phi)(\beta):=\phi \beta \phi^{-1}=\exp (a d \gamma) \beta .
$$

temos que a relação $A d \exp =\exp a d$ é verdadeira como se espera em qualquer endomorfismos em que ambos os lados convergem.

Graças a esta relação, com um pouco de trabalho algébrico, temos como consequência algumas identidades que serão enunciadas abaixo. 


$$
\begin{gathered}
a d \gamma\left(\iota_{b} \otimes 1\right)=-1 \otimes \iota_{b} \\
a d \gamma\left(\nu \otimes \iota_{b}\right)=0 \forall \nu \in A \\
(a d \gamma)^{2}\left(\iota_{b} \otimes 1\right)=0 \\
a d \gamma(d)=-d \theta^{a} \otimes \iota_{a}+\theta^{a} \otimes \mathcal{L}_{a} \\
(a d \gamma)^{2}(d)=-c_{a b}^{k} \theta^{a} \theta^{b} \\
(a d \gamma)^{3} d=0
\end{gathered}
$$

Caso o leitor fique interessado, a prova destas identidades está no apêndice $\mathrm{C}$.

O mapa que iremos usar para levar o modelo de Weil no modelo de Cartan é o $A d(\phi)$. Ele, como veremos, leva $(A \otimes B)_{h o r}$ em $A_{\text {hor }} \otimes B$. Como falamos no início do capítulo, teremos algum tipo de perda, isto é, o operador $d$ perderá simplicidade. Vejamos então como $A d(\phi)$ age em $\bar{g}$.

Teorema 7.1.1 $\left[\gamma, \mathcal{L}_{a} \otimes 1+1 \otimes \mathcal{L}_{a}\right]=0$

Prova:

$$
\left[\gamma, \mathcal{L}_{a} \otimes 1+1 \otimes \mathcal{L}_{a}\right]=a d \gamma\left(\mathcal{L}_{a} \otimes 1\right)+a d \gamma\left(1 \otimes \mathcal{L}_{a}\right)
$$

Ora, mas

$$
\left[\theta^{b} \otimes \iota_{b}, \mathcal{L}_{a} \otimes 1\right]=\left[\theta^{b}, \mathcal{L}_{a}\right] \otimes \iota_{b}=-c_{a k}^{b} \theta^{k} \otimes \iota_{b}
$$

$\mathrm{e}$

$$
\begin{aligned}
& {\left[\theta^{b} \otimes \iota_{b}, 1 \otimes \mathcal{L}_{a}\right]=\theta^{b} \otimes\left[\iota_{b}, \mathcal{L}_{a}\right]=\theta^{b} \otimes-c_{b a}^{k} \iota_{k}=-c_{b a}^{k} \theta^{b} \otimes \iota_{k}=c_{a k}^{b} \theta^{k} \iota_{b} .} \\
& \operatorname{Logo}\left[\gamma, \mathcal{L}_{a} \otimes 1+1 \otimes \mathcal{L}_{a}\right]=0 .
\end{aligned}
$$

Assim, como $\operatorname{Ad}(\phi)\left(\mathcal{L}_{a}\right)=\phi \mathcal{L}_{a} \phi^{-1}=\exp (a d \gamma) \mathcal{L}_{a} \exp (a d \gamma) \mathcal{L}_{a}=\mathcal{L}_{a}$ pelo que fizemos acima, temos que:

\section{Corolário 7.1.2}

$$
\phi \mathcal{L}_{a} \phi^{-1}=\mathcal{L}_{a}
$$




\section{Teorema 7.1.3}

$$
\phi\left(1 \otimes \iota_{\xi}+\iota_{\xi} \otimes 1\right) \phi^{-1}=\iota_{\xi} \otimes 1 \forall \xi \in g .
$$

Repare que este teorema diz que uma forma horizontal em $A \otimes B$ é levada em $A_{\text {hor }} \otimes B$.

Prova: Tomando $\xi=\xi_{b}$, repare que por 7.1.5 e 7.1.6, $(\exp a d \gamma)\left(1 \otimes \iota_{\xi}+\iota_{\xi} \otimes 1\right)$ não tem grau maior que 1 na expansão, isto é,

$$
(\exp a d \gamma)\left(1 \otimes \iota_{\xi}+\iota_{\xi} \otimes 1\right)=\left(1 \otimes \iota_{b}+\iota_{b} \otimes 1\right)+(a d \gamma)\left(1 \otimes \iota_{b}+\iota_{b} \otimes 1\right)
$$

Assim, usando 7.1.4 e 7.1.6, temos que

$$
(\exp a d \gamma)\left(1 \otimes \iota_{\xi}+\iota_{\xi} \otimes 1\right)=\left(1 \otimes \iota_{b}+\iota_{b} \otimes 1\right)-1 \otimes \iota_{b}=\iota_{b} \otimes 1
$$

Falta agora verificar como fica o operador $d$.

\section{Teorema 7.1.4}

$$
\phi d \phi^{-1}=d-\mu^{k} \otimes \iota_{k}+\theta^{k} \otimes \mathcal{L}_{k} .
$$

Prova: Por 7.1.9, a expansão da exponencial não tem termo de grau maior que 2, ou seja,

$$
\phi d \phi^{-1}=d+(a d \gamma) d+\frac{1}{2}\left(a d_{\gamma}\right)^{2} d
$$

E, então, por 7.1 .7 e 7.1 .8

$$
\phi d \phi^{-1}=d-d \theta^{k} \otimes \iota_{k}+\theta^{k} \otimes \mathcal{L}_{k}-\frac{1}{2} c_{a b}^{k} \theta^{a} \theta^{b} \otimes \iota_{k}=d-\mu^{k} \otimes \iota_{k}+\theta^{k} \otimes \mathcal{L}_{k} .
$$

Repare ainda que, como $\gamma$ é uma derivação, $\exp \gamma$ é uma derivação, em particular uma derivação de grau 0 , como vimos. Desta forma, caso $A$ seja uma $W^{*}$-álgebra e $B$ uma $G^{*}$-álgebra, como a operação de produto é mantida por ser uma derivação, temos que $\phi$ é um automorfismo de álgebras.

\section{2}

\section{Modelo de Cartan}

Na seção anterior mostramos que $A d(\phi)$ faz uma passagem do subespaço horizontal de $A \otimes B$ para $A_{\text {hor }} \otimes B$. Nesta seção iremos comprender mais esta 
passagem, incluindo o subespaço $A_{\text {hor }} \otimes B$, afim de representar o modelo de Cartan. Faremos um abuso de notação, chamaremos $A d(\phi)$ simplesmente por $\phi$ e não representaremos explicitamente o mapa com a restrição, como é mais conveniente. Então temos

$$
\phi:(A \otimes B)_{h o r} \rightarrow A_{h o r} \otimes B .
$$

Voltemos então para o caso $A=W$. Construímos no capítulo anterior uma teoria muito forte da álgebra de Weil, então sabemos muito sobre ele. Para começar, em vez do abstrato $A_{\text {hor }}$, o $W_{\text {hor }}$ é um conjunto muito mais "concreto", pois temos a igualdade

$$
W_{h o r}=\mathbb{C}\left[\mu^{1}, \ldots, \mu^{n}\right] \cong S\left(g^{*}\right) .
$$

Com o que fizemos na seção anterior fica claro como o operador $\iota_{a}$ e o operador $\mathcal{L}_{a}$ se comportam neste espaço, o mesmo não acontece com $d$, apesar dele ter uma fórmula explícita é um pouco difícil entender o que está acontecendo. Neste sentido a álgebra de Weil também vai nos ajudar. Graças a equação $6.1 .9, d=d_{W}$ quando restrita às formas horizontais é igual a $\theta^{a} \mathcal{L}_{a}$. Agora, considerando $d=d_{W} \otimes 1+1 \otimes d_{B}$ como estamos considerando, podemos reescrever a equação 7.1 .11 como

$$
\phi(d)=\left(\theta^{a} \otimes 1\right)\left(\mathcal{L}_{a} \otimes 1+1 \otimes \mathcal{L}_{a}\right)+1 \otimes d_{B}-\mu^{a} \otimes \iota_{a}
$$

Assim, como $\phi$ comuta com $\mathcal{L}_{a}$ por 7.1.2, temos que $\phi$ é $G$-equivariante e, portanto, ao restringirmos $\phi$ aos elementos invariantes, temos o isomorfismo

$$
\phi:(W \otimes B)_{b a s} \rightarrow\left(W_{h o r} \otimes B\right)^{G}=\left(S\left(g^{*}\right) \otimes B\right)^{G} .
$$

E assim, quando restrito a elementos invariantes temos

$$
\phi(d)=1 \otimes d_{B}-\mu^{a} \otimes \iota_{a}
$$

Ou seja, $1 \otimes d_{B}-\mu^{a} \otimes \iota_{a}$ é a derivação que representa o operador $d$ em $\left(S\left(g^{*}\right) \otimes B\right)^{G}$.

Assim definimos:

Definição 7.2.1 Seja B um $G^{*}$-módulo. O espaço

$$
C_{G}(B):=\left(S\left(g^{*}\right) \otimes B\right)^{G}
$$

munido com a diferencial 


$$
d_{G}: C_{G}(B) \rightarrow C_{G}(B), 1 \otimes d_{B}-\mu^{a} \otimes \iota_{a}
$$

é o chamado modelo de Cartan para a cohomologia equivariante de B.

Assim, provamos o teorema fundamental de Cartan:

Teorema 7.2.2 O mapa $\phi$ leva $(W \otimes B)_{\text {bas }}$ em $C_{G}(B)$ e leva a restrição de d a $d_{G}$. Logo

$$
H^{*}\left(\left((W \otimes B)_{b a s}\right), d\right)=H^{*}\left(C_{G}(B), d_{G}\right) .
$$

Um ponto interessante no modelo de Cartan é que ele nos permite ver de uma forma diferente seus elementos, isto é, um elemento $\omega \in C_{G}(B)$ pode ser visto como um mapa polinomial equivariante de $g$ a $B$. Na verdade isto se deve também a $S\left(g^{*}\right)$ que, como foi apresentado, pode ser visto como polinômios com variáveis em $g$. Vejamos de uma forma mais explícita.

Seja $\omega \in C_{G}(B)$. Então

$$
\omega=\sum_{i} p_{i} \otimes b_{i}
$$

onde $p_{i} \in S\left(g^{*}\right)$ e $b_{i} \in B$. Repare que $p_{i} \in S\left(g^{*}\right)$ pode ser escrito como:

$$
p_{i}=\lambda_{0}+\lambda_{11} x_{1}+\lambda_{12} x_{2}+\ldots+\lambda_{212} x_{1} x_{2}+\ldots+\lambda_{n} x_{1} \ldots x_{n}
$$

onde $\lambda_{\text {.. }} \in \mathbb{C}$ e $x_{1}, \cdots, x_{n} \in g^{*}$ é a base dual de $\xi_{1}, \cdots, \xi_{n} \in g$. Então

$$
p_{i}(\xi)=\left(\lambda_{0}+\lambda_{11} x_{1}(\xi)+\ldots+\lambda_{n} x_{1}(\xi) \cdot \ldots \cdot x_{n}(\xi)\right) \in \mathbb{C} .
$$

Assim temos o mapa de polinomial (que será equivariante porque o elemento é equivariante) de $g$ em $B$ dado por:

$$
\sum_{i} p_{i} \otimes b_{i}: g \rightarrow B, \quad \xi \mapsto p_{i}(\xi) b_{i}
$$

Com esta interpretação, o elemento $\left(\mu^{a} \otimes \iota_{a}\right) \omega$ é o mapa

$$
\xi \mapsto \mu^{\xi} \iota_{\xi} \omega(\xi)
$$

pois $\xi=\bar{\lambda}_{1} \xi_{1}+\ldots+\bar{\lambda}_{n} \xi_{n}$ para alguns $\bar{\lambda}_{1}, \cdots, \bar{\lambda}_{n} \in \mathbb{C}$ únicos.

Assim, caso $\omega$ seja um polinômio homogêneo, $\xi \mapsto \mu^{\xi} \iota_{\xi} \omega(\xi)$ é um mapa polinômial de grau 1 pois estaremos multiplicando por $\mu^{\xi}$. Assim, na graduação de $S\left(g^{*}\right)$ e $B$ usuais, temos que se

$$
\omega \in S^{k} \otimes B_{l}
$$


então

$$
\mu^{a} \otimes \iota_{a} \omega \in S^{k+1} \otimes B_{l-1}
$$

E, em particular, como o grau de $S^{k}$ é igual a $2 k$, temos que o grau total $2 k+l$ é acrescido em 1.

Então, do ponto de vista de mapas polinomiais, o operador $d_{G}$ está bem definido como

$$
d_{G}(\omega)(\xi)=d_{B}[\omega(\xi)]-\mu^{\xi} \iota_{\xi}[\omega(\xi)]
$$

e tem grau 1.

O propósito inicial do modelo de Cartan é provar que a definição da cohomologia equivariante independe da escolha da $W^{*}$-álgebra acíclica escolhida. Então, aqui teríamos dois caminhos a serem escolhidos para provar o mesmo teorema (teorema 7.3.1). O primeiro usa a estrutura polinomial de $C_{G}(B)$ e, a partir disto, cria alguns operadores que, de uma certa perspectiva, são mais geométricos por estar trabalhando com polinômios. A prova, neste contexto, também utiliza uma decomposição simultânea em autoespaços de $C_{G}(B)$ a partir destes operadores. Esta prova é muito linda e com ela fica claro que a cohomologia independe da estrutura específica de $W^{*}$-módulo que é dada a um $W^{*}$-módulo. Esta prova pode ser encontrada no capítulo 5 de [GS].

No caso deste trabalho seguiremos por outro caminho.

\section{3}

\section{Cohomologia Equivariante de $W^{*}$-módulos}

Antes de começar a dissertar sobre a Cohomologia equivariante de $W^{*}$-módulos relembremos algo que já usamos nesta dissertação, o processo de fazer a média. Este processo usa que a medida de Haar é invariante. Assim, se considerarmos $G$ um grupo de Lie compacto, podemos transformar certos tipos de funcionais suaves em funcionais suaves $G$-invariantes. Como vimos antes, esta construção é válida para métricas riemanianas, porém, além desta, também é válida para formas diferenciáveis e a construção é bem semelhante.

Como usaremos o resultado acima iremos consideraremos $G$ um grupo de Lie compacto. É claro que fazer isto também permite, entre outras coisas, representarmos $M / G$ como uma variedade, no caso de $M$ variedade e a ação de $G$ ser livre. Na hipótese de $M$ também ser compacto, como podemos ver pelo teorema equivariante de de Rham, temos a igualdade entre a cohomologias equivariante topológica e a de $G^{*}$-módulos. 
Repare que no decorrer do texto fazemos algumas vezes a suposição de $G$ ser compacto ou conexo, isto não leva a nenhuma perda para o nosso resultado, já que, isso faz e fará parte das nossa hipóteses. Não fazemos esta suposição sempre para manter a generalidade dos resultados.

Então, considerando $G$ compacto, provemos:

Teorema 7.3.1 Sejam $A$ um $W^{*}$-módulo e $E$ uma $W^{*}$-álgebra acíclica. Então

$$
H^{*}\left((A \otimes E)_{b a s}\right)=H^{*}\left(A_{b a s}\right)
$$

Consideremos o isomorfismo de Mathai-Quillen e $B=E$ uma $W^{*}$-álgebra acíclica. Assim

$$
\phi:(A \otimes E)_{b a s} \rightarrow\left(A_{h o r} \otimes E\right)^{G}
$$

quando restringimos $\phi$ às formas $G$-equivariantes. $O$ operador transforma $d$ em $(A \otimes E)_{b a s}$ no operador, que chamaremos de $\delta, d_{A} \otimes 1+1 \otimes d_{E}+\theta^{a} \otimes \mathcal{L}_{a}-\mu^{a} \otimes \iota_{a}$ em $\left(A_{\text {hor }} \otimes E\right)^{G}$.

Considere então

$$
\delta_{1}:=1 \otimes d_{E}
$$

$\mathrm{e}$

$$
\delta_{2}:=d_{A} \otimes 1+\theta^{a} \otimes \mathcal{L}_{a}-\mu^{a} \otimes \iota_{a} .
$$

Temos que $\delta=\delta_{1}+\delta_{2}$.

Defina:

$$
C^{i}:=\left(A_{h o r} \otimes E^{i}\right)^{G} \text { e } C^{*}:=\oplus C^{i} .
$$

É fácil ver que $\delta_{1}$ acresce o grau de $E$ em 1 e, enquanto os dois primeiros elementos de $\delta_{2}$ mantêm o grau de $E$, o último elemento de $\delta_{2}$ decresce o grau de $E$ em 1. Portanto:

$$
\delta_{1}: C^{i} \rightarrow C^{i+1}
$$

e

$$
\delta_{2}: C^{i} \rightarrow C^{i}+C^{i-1} .
$$

Além disso, pela definição de $d_{E}, \delta_{1}^{2}=0$, logo podemos considerar $\delta_{1}$ como o operador diferencial no complexo $C^{*}$ e com isto estudar a cohomologia de $C^{*}$ em relação a $\delta_{1}$. 
Ora, mas considere $\bar{C}^{i}:=A_{\text {hor }} \otimes E^{i}$ e $\bar{C}^{*}:=\oplus \bar{C}^{i}$. Repare que $C^{i} \in \bar{C}^{i}$. Como $E$ é, por hipótese, acíclico,

$$
H^{0}\left(\bar{C}, 1 \otimes d_{E}\right)=A_{h o r}
$$

e, se $k>0$,

$$
H^{k}\left(\bar{C}, 1 \otimes d_{E}\right)=0
$$

Assim, se $k>0$, para todo $\tau \in C^{k}$ que satisfaz $d \tau=0$, existe $\alpha \in \bar{C}^{k-1}$ tal que $\left(1 \otimes d_{E}\right) \alpha=\tau$. Como $G$ é compacto, podemos fazer a média e assumir que $\alpha$ é $G$ - invariante e, portanto, $\alpha$ estará em $C^{k-1}$.

Logo os grupos de cohomologia de $\left(C_{1}^{*}, \delta_{1}\right)$ são dados por

$$
H^{0}\left(C^{*}, \delta_{1}\right)=A_{\text {bas }}
$$

e, se $k>1$,

$$
H^{k}\left(C^{*}, \delta_{1}\right)=0
$$

Talvez agora seja interessante apresentar a definição e fazer alguns comentários a respeito de filtração, pois iremos construir uma na sequência e também é usado no apêndice D.

Definição 7.3.2 Dada uma álgebra A, uma filtração crescente (decrescente) é uma coleção $\mathcal{F}=\left\{F_{n}\right\}_{n \in \mathbb{Z}}$ com $F_{n} \subset A$ tal que $F_{n} \subset F_{n+1}\left(F_{n+1} \subset F_{n}\right) e$ $F_{n} \cdot F_{m} \subset F_{n+m}\left(F_{n} \cdot F_{m} \subset F_{-|n-m|}\right)$.

Desta forma, temos que $\operatorname{gr}(A, \mathcal{F})=\oplus F_{n} / F_{n-1}\left(\operatorname{gr}(A, \mathcal{F})=\oplus F_{n-1} / F_{n}\right)$ é uma álgebra graduada.

Vale ressaltar que, caso $A$ já seja uma álgebra graduada, temos que $F_{n}=\bigoplus_{j \leq n} A_{j}$ já representa uma filtração e, ainda, neste caso temos que $F_{n} / F_{n-1} \cong A_{n}$ e, portanto, $A \cong g r(A, \mathcal{F})$ como álgebras graduadas.

Consideremos então

$$
C_{j}=\bigoplus_{i \leq j} C^{i}
$$

tal que

$$
C_{j} \subset C_{j+1},\left(A_{h o r} \otimes E\right)^{G}=C^{*}=\bigcup C_{j}
$$

gera uma filtração crescente de $\left(A_{\text {hor }} \otimes E\right)^{G} \operatorname{com} C_{0}=A_{\text {bas }}$. 
Estamos agora mais preparados para provar o teorema 7.3.1.

Prova: [de 7.3.1]

Temos que mostrar que a classe dos elementos em $(A \otimes E)_{b a s}$ só depende de $A_{\text {bas }}$, isto é, se $\mu \in C^{i}$ tal que $d \mu=0$ então $\mu$ pode ser escrito como a soma de um elemento em $A_{\text {hor }}$ por um elemento na imagem de $\delta$.

Então é suficiente provar o seguinte lema:

Lema 7.3.3 Se $\mu \in C_{j}$ satisfaz que $\delta \mu=0$, existe um $\nu \in C_{j-1}$ e um elemento em $a \in A_{b a s}$ tal que

$$
\mu=\delta \nu+a \otimes 1 \text {. }
$$

Além disso, a é único a menos de fronteira de $A_{b a s}$, isto é, se $\mu=0$ então $a=d_{A} b$, onde $b \in A_{b a s}$

O lema é interessante porque já nos induz um processo indutivo em cima de $j$. Ora, mas em grau 0 a construção é consequência da cohomologia de $\left(C^{*}, \delta_{1}\right)$ que construímos antes, isto é, se $j=0$ então $\delta \mu=0$ implica $\delta_{1} \mu=0$. Ou seja, pela cohommologia de $\left(C^{*}, \delta\right), \mu \in A_{b a s}$.

Assim, se $\nu \in$ satisfaz a hipótese, por $\delta_{1}$ ser de grau 1 , temos que $\delta_{1} \nu=0$. Assim $\nu \in A_{b a s} \otimes 1$, então $\nu=-b \otimes 1$ para algum $b \in A_{b a s}$. Segue então que

$$
\delta_{2} \nu=\left(d_{A} \otimes 1\right) \nu+\theta^{j} \otimes \mathcal{L}_{j} \nu-\mu^{j} \otimes \iota_{j} \nu=-\left(d_{A} b\right) \otimes 1 .
$$

Portanto, como queríamos,

$$
a=d_{A} b .
$$

Façamos agora a hipótese de indução, suponha que o lema é válido para $j-1$ com $j \geq 1$. Seja $\mu \in C_{j} \operatorname{com} \delta \mu=0$. Mas $C_{j}=\oplus C^{i}, \operatorname{logo}$

$$
\mu=\mu_{j}+\mu_{j-1}+\ldots
$$

onde $\mu \in C_{j}$ enquanto $\mu-\mu_{j}=\mu_{j-1}+\ldots \in C^{j-1}$. Ora, mas $\delta \mu=0$, logo $\delta_{1} \mu=0$. Mas para $j>0, H^{j}\left(C^{*}, \delta_{1}\right)=0$, segue então que $\mu=\delta_{1} \nu_{j-1}$.

Logo,

$$
\mu=\delta \nu_{j-1}+\omega
$$

onde $\omega \in C_{j-1}$. Como $\delta \mu=0$ e $\delta^{2}=0$ temos que $\delta \omega=0$. Assim, como o resultado vale para $j-1$ segue-se o resultado para $j$. 
Com isto finalmente terminamos a prova do teorema 7.3.1. Vale ressaltar que o isomorfismo entre $H_{\text {bas }}(A)$ e $H_{G}(A)$ depende apenas da estrutura como $G^{*}$-módulo e não da estrutura como $W^{*}$-módulo.

Assim, como pelo teorema 6.2.1, uma $E$ álgebra do tipo (C) é uma $W^{*}$ álgebra, temos que dado $E$ e $F$ duas $G^{*}$-álgebras do tipo $(\mathbf{C})$, ou simplesmente $W^{*}$-álgebras, que são acíclicas, a seguinte igualdade é verdadeira:

$$
H_{\text {bas }}^{*}(A \otimes F)=H_{b a s}^{*}(A \otimes F \otimes E)=H_{b a s}^{*}(A \otimes E) .
$$

Portanto, a definição da cohomologia equivariante $H_{G}(A)$ é perfeita. Dada a construção que fizemos até aqui, como a álgebra de Weil é a $G^{*}$ álgebra mais simples que satisfaz a condição $(\mathbf{C})$, pode ser interessante entender $H_{G}^{*}(A)$ como $H_{b a s}^{*}(A \otimes W)$, ou simplesmente, graças ao teorema fundamental de Cartan, $H\left(C_{G}(A), d_{G}\right)$.

\section{4 \\ O Homomorfismo Característico}

Com a construção que fizemos neste capítulo, temos a oportunidade de costruir uma versão diferente do mapa de Chern-Weil, tanto para $G^{*}$-álgebras como para variedades. Comecemos então com $G^{*}$-álgebras.

Seja $A$ uma $G^{*}$-álgebra com identidade 1 . Podemos considerar $\mathbb{C}$ como $G^{*}$-módulo trivial e como $\mathbb{C} \otimes W=W$, vemos a cohomologia equivariante de $\mathbb{C}$ da forma:

$$
H_{G}(\mathbb{C})=H_{b a s}(W)=S\left(g^{*}\right)^{G} .
$$

Consideremos então o mapa

$$
\kappa: \mathbb{C} \rightarrow A, z \mapsto z 1_{A}
$$

Como o mapa é um homomorfismo de $G^{*}$-módulos, já que $\mathbb{C}$ é uma $G^{*}$-módulo trivial, temos que ele induz um homomorfismo na cohomologia equivariante:

$$
\kappa_{G}: S\left(g^{*}\right)^{G} \rightarrow H_{G}(A)
$$

E temos novamente o mapa de Chern-Weil. Os elementos da imagem de $\kappa_{G}$ são conhecidos como as classe de caracteística de $H_{G}(A)$. 
Agora considere $M$ uma variedade em que $G$ age livremente e $A=\Omega(M)$. Consideremos o mapa constante

$$
M \rightarrow \text { ponto }
$$

Como um ponto é uma variedade, em particular de dimensão 0, podemos considerar o mapa pullback no nível das formas e, portanto, pensando em $G$ agindo no ponto trivialmente, temos o mapa induzido na cohomologia equivariante

$$
H_{G}^{*}(\text { ponto }) \rightarrow H_{G}^{*}(M)
$$

Agora, como por hipótese $G$ age livremente em $M$, definindo $X=M / G$, temos que

$$
H_{G}^{*}(M)=H^{*}(X)
$$

Além disso, como

$$
H_{G}^{*}(\text { ponto })=H_{G}(\Omega(\text { ponto }))=H_{G}(\mathbb{C})=S\left(g^{*}\right)^{G},
$$

podemos identificar o mapa de Chern-Weil como o mapa

$$
S\left(g^{*}\right)^{G} \rightarrow H^{*}(X)
$$

A algumas seções atrás, comentamos que no capítulo 5 de [GS], Guillemmin e Sternberg fazem construções muito interessantes sobre o modelo de Cartan, onde, inclusive, provam o teorema 7.3.1 de uma forma um pouco diferente. A construção se inicia ao identificar $S\left(g^{*}\right) \otimes A$ com o espaço polinomial de valores em $A$. Neste caso, associamos a base $\xi_{1}, \ldots, \xi_{n}$ de $g$ com coordenadas correspondentes $x_{1}, . ., x_{n}$. Assim, usa-se $x_{i}$ em vez do clássico $\mu^{i}$ em $W(g)$ e, então, um elemento da forma $\sum x^{I} \otimes a_{I}$ é representado por $\sum a_{I} x^{I}$ e a diferencial do modelo de Cartan é dada por $\left(d_{G} \alpha\right)(x):=d[\alpha(x)]-x^{r} \iota^{r}[\alpha(x)]$.

Introduziremos esta notação neste momento para entender um pouco mais da imagem do mapa de Chern Weil no caso de variedades. Esta construção pode ser vista de forma mais precisa em [GS] e toma como base o mapa avaliação:

$$
\left(S\left(g^{*}\right) \otimes A\right)^{G} \rightarrow A_{b a s}, x^{I} \otimes a \mapsto \mu^{I} a .
$$

Consideremos o $G$-fibrado principal $\pi: M \rightarrow X$ que atribui a cada ponto $m \in M$ sua $G$-órbita. Então, atribuindo a este fibrado seus elementos de conexão

$$
\mu^{i} \in \Omega(M)_{h o r}
$$


e um mapa $G$-equivariante

$$
\rho: S\left(g^{*}\right)^{G} \otimes \Omega(M) \rightarrow \Omega(M)_{h o r}, \quad x^{I} \otimes \omega \mapsto \mu^{I} \wedge \omega_{h o r} .
$$

De $\pi$ obtemos o isomorfismo

$$
\pi^{*}: \Omega(X) \rightarrow \Omega(M)_{b a s}
$$

Então, compondo $\rho$ restrito aos elementos invariantes com o mapa inverso de $\pi^{*}$, obtemos o mapa

$$
\left(S\left(g^{*}\right) \otimes \Omega(M)\right)^{G} \rightarrow \Omega(X) .
$$

A restrição deste mapa a $S\left(g^{*}\right)$ é o mapa de Chern-Weil no nível de formas e induz um mapa na cohomologia

$$
S\left(g^{*}\right)^{G} \rightarrow H^{*}(X)
$$

Então, para

$$
p=p\left(x_{1}, \ldots, x_{n}\right) \in S\left(g^{*}\right)^{G}
$$

sua imagem pelo homomorfismo característico é

$$
p\left(\mu^{1}, \ldots, \mu^{n}\right) .
$$

Este mapa está bem definido e independe da escolha de conexão. A construção acima está contida e explicada no capítulo 5 de [GS]. O importante no nosso caso é ver que a imagem pelo mapa característico de um polinômio em $S\left(g^{*}\right)^{G}$ pode ser visto como um polinômio, de forma semelhante (só trocando as variáveis), em $H^{*}(X)$.

\section{5}

\section{Cohomologia Equivariante de Espaços Homogêneos}

A teoria que fizemos até aqui nos permite ter, com um pouco de trabalho, explicitamente a cohomologia equivariante de espaços homogêneos. Para chegar neste resultado estudemos um pouco sobre ações que comutam.

Considere $G=P \times K$, onde $P$ e $K$ são grupos de Lie. Pela teoria de grupos de Lie, sabemos que a álgera de Lie de $G$ pode ser escrita como

$$
g=p \oplus k,
$$


onde $p$ e $k$ são as álgebras de Lie de $P$ e $K$ respectivamente. E, portanto,

$$
g^{*}=p^{*} \oplus k^{*}
$$

Ora, mas sabemos, por exemplo, que $\wedge\left(p^{*} \oplus k^{*}\right)=\wedge\left(p^{*}\right) \otimes \wedge\left(k^{*}\right)$, logo

$$
W(g)=W(p) \otimes W(k)
$$

e $d_{W(g)}=d_{W(p)} \otimes 1_{K}+1_{P} \otimes d_{W(k)}$.

Agora, como $g=p \oplus k$, também podemos pensar em $\bar{p}$ e $\bar{k}$ como subálgebras de $\bar{g}$. Mas observe que, dados $x \in P$ e $y \in K$, temos que

$$
A d(x \otimes 1)(1 \otimes y)=(x \otimes 1)(1 \otimes y)\left(x^{-1} \otimes 1\right)=(1 \otimes y)
$$

e, portanto,

$$
\left[\mathcal{L}_{\xi_{x}}, \mathcal{L}_{\xi_{y}}\right]=0 \quad \text { e } \quad\left[\iota_{\xi_{x}}, \iota_{\xi_{y}}\right]=0
$$

Logo, $p_{-1} \oplus p_{0}$ comuta com $k_{-1} \oplus k_{0}$.

Assim, como um $G^{*}$-módulo, $A$ pode ser visto também como um $K^{*}$-módulo ou $P^{*}$-módulo, temos que

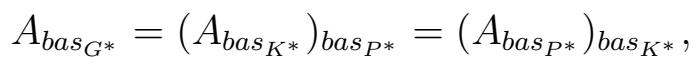

onde $A_{b a s_{G^{*}}}$, por exemplo, representa os elementos básicos de $A$ para a $G^{*}$ ação. O resultado é válido porque as ações de $P^{*}$ e $K^{*}$ comutam e podemos ver $A_{b a s_{P^{*}}}$ como um submódulo para a $K^{*}$ ação ou vice e versa.

Agora, aplicando isto a $A \otimes W(g)=A \otimes W(p) \otimes W(k)$, supondo que $A$ e, portanto, $A \otimes W(p)$ sejam $(W(k))^{*}$-módulos, temos, pelo teorema 7.3.1, que

$$
\begin{aligned}
& H_{G}(A):= \\
& =H\left((A \otimes W(p) \otimes W(k))_{\text {bas }_{G^{*}}}, d\right)= \\
& \text { (onde } d=d_{A} \otimes 1_{W(p)} \otimes 1_{W(k)}+1_{A} \otimes d_{p} \otimes 1_{W(k)}+1_{A} \otimes 1_{W(p)} \otimes d_{k} \text { ) } \\
& =H\left(\left((A \otimes W(p) \otimes W(k))_{b a s_{P^{*}}}\right)_{b a s_{K^{*}}},\left(d_{A} \otimes 1_{W(p)}+1_{A} \otimes d_{p}\right) \otimes 1+1 \otimes d_{K}\right)= \\
& =H\left(\left(A_{b a s_{K^{*}}} \otimes W(p)\right)_{b a s_{P^{*}}}, d_{A} \otimes 1_{W(p)}+1_{A} \otimes d_{p}\right)= \\
& =: H_{P}\left(A_{b a s_{K^{*}}}\right) \text {. }
\end{aligned}
$$




$$
H_{G}(A)=H_{P}\left(A_{b a s_{K^{*}}}\right)
$$

Portanto, caso $A$ também seja um $(W(p))^{*}$-módulo, concluímos que

$$
H_{K}\left(A_{b a s_{P^{*}}}\right)=H_{G}(A)=H_{P}\left(A_{b a s_{K^{*}}}\right) .
$$

Com este resultado, podemos estudar a cohomologia equivariante do espaço homogêno $G / K$. De fato, consideremos $K$ um subgrupo fechado de $G$, logo também um grupo de Lie. Podemos considerar uma ação de $G \times K$ em $G$, onde $G$ age em si mesmo pela esquerda e $K$ age em $G$ pela direita. Assim temos que:

$$
H_{G \times K}(G)=H_{G}(G / K)=H_{K}(G / G)=H_{K}(\text { ponto })=S\left(k^{*}\right)^{K}
$$

e, portanto, $S\left(k^{*}\right)^{K}$ é a cohomologia equivariante do espaço homogêneo $G / K$.

Estudemos um pouco mais de ações que comutam. Consideremos agora que $P$ seja compacto e, então, podemos fazer a média sobre ele e representar a equação 7.5.1 pelo modelo de Catan. Isto é, considere as formas de conexão $\theta^{i}$ tais que definem $A$ como um $(W(k))^{*}$-módulo para a ação de $K^{*}$. Assim, como $K$ e $P$ comutam, calculando a média dos $\theta^{i}$ 's sobre $P$ obtemos aqueles que são $M$ invariantes. Logo, assumindo que $\left\{\eta_{1}, \ldots, \eta_{r}\right\}$ é a base de $k$ e $\left\{\nu_{1}, \ldots, \nu_{r}\right\}$ sua base dual, temos

$$
C_{G}(A)=\left(S\left(K^{*}\right) \otimes\left(S\left(P^{*}\right) \otimes A\right)^{P}\right)^{K}
$$

e, usando a equação 7.2.6,

$$
d_{C(G)}=d_{C(K)}\left(d_{C(P)}\right)=1 \otimes d_{C(P)}-\nu^{j} \iota\left(\eta_{j}\right)
$$

onde $d_{C(K)}$ e $d_{C(P)}$ são os operadores $d$ no modelo de Cartan relativo, respectivamente, a $K^{*}$ e a $P^{*}$.

Em particular, esta cohomologia é isomorfa a cohomologia relativa a $d_{C(P)}$ de

$$
\left(S\left(p^{*}\right) \otimes A_{b a s_{K^{*}}}\right)^{P}
$$

que é $H_{P}\left(A_{b a s_{K^{*}}}\right)$ como vimos.

Neste caso, a imagem do mapa de Chern-Weil

$$
\kappa_{K}: S\left(k^{*}\right)^{K} \rightarrow H_{P}\left(A_{b a s_{K^{*}}}\right)
$$

é chamado o anel de classes de característica equivariante de $P$. 


\section{8 \\ Cohomologia do Fibrado Flag Homogêneo}

Neste capítulo iremos finalmente provar o resultado principal desta dissertação, uma fórmula explícita para calcular a cohomologia do fibrado flag homogêneo.

Durante boa parte deste trabalho focamos na teoria equivariante, principalmente na teoria equivariante de $G^{*}$-módulos. Esta é uma bela teoria que não se limita a provar nosso resultado, o cálculo da cohomologia do fibrado flag homogêneo é apenas uma de suas aplicações. Porém, nos limitaremos a isto. Para ficar mais claro o caminho que fizemos até agora talvez seja interessante fazer um breve resumo.

Primeiro apresentamos alguns conceitos iniciais das estruturas algébricas e topológicas que trabalhamos nesta dissertação e, rapidamente, apresentamos uma motivação para o resultado que buscamos. Nesta motivação discutimos sobre a cohomologia equivariante topológica, que, sobre algumas hipóteses, equivale a cohomologia equivariante de $G^{*}$-módulos. Então surge a pergunta: Por que escolhemos especificamente $G^{*}$-módulos e a cohomologia equivariante de $G^{*}$-módulos como objeto de estudo? Ao longo do texto pudemos ver que a teoria inicial no caso de $G^{*}$-módulos exige mais trabalho do que o caso topológico, então por que a escolhemos?

A resposta disto é a liberdade e a mecânica algébrica que ganhamos. Antes de construirmos a estrutura de $G^{*}$-módulos, provamos as identidades geométricas consequentes da ação de um grupo de Lie $G$ sobre uma variedade $M$. Desta forma, exigindo que um $G^{*}$-módulo satisfaça estas propriedades, construímos um conceito mais geral e puramente algébrico. Ainda neste sentido, a condição (C) vem justamente algebrizar de forma a podermos trabalhar no modelo infinitesimal o conceito de ação livre, ou melhor dizendo, localmente livre.

Posteriormente vimos a álgebra de Weil, a $G^{*}$-álgebra acíclica mais simples que satisfaz a condição $(\mathbf{C})$. Se anteriormente não estava muito claro 
o porquê de trabalharmos com a cohomologia equivariante de $G^{*}$-módulos, a álgebra de Weil sintetiza o motivo da escolha. A álgebra de Weil tem uma estrutura muito bem definida e razoavelmente simples de se trabalhar e, então, junto com isomorfismo de Mathai-Quillen e o modelo de Cartan, provamos que é boa, no sentido de estar bem definida, a definição de cohomologia equivariante de $G^{*}$-módulos.

Entretanto, a beleza da álgebra de Weil e do modelo de Cartan não acaba por aí, neste capítulo mostraremos que o modelo de Cartan admite uma estutura de complexo duplo. Assim, usando um pouco de teoria de sequência espectral que está no apêndice D e com algum trabalho, estenderemos mais a teoria adquirindo resultados que finalmente nos permitira gerar uma fórmula explícita para calcular a Cohomologia do Fibrado Flag Homogêneo.

\section{1}

\section{Modelo de Cartan, um Duplo Complexo}

Como dissemos na introdução deste capítulo, iremos usar um pouco da teoria espectral de complexo duplo, teoria esta que está brevemente apresentada no apêndice D. Convido o leitor, caso tenha interesse, a ver este capítulo no apêndice, ou, pelo menos, dar uma breve olhada, pois usaremos definições, conceitos e notações lá presentes.

Consideremos novamente $G$ um grupo de Lie compacto e $A=\underset{k \in \mathbb{Z}}{\oplus} A^{k}$ um $G^{*}$-módulo. Seu complexo de Cartan é dado por

$$
C_{G}(A)=\left(S\left(g^{*}\right) \otimes A\right)^{G} .
$$

Iremos considerar o complexo duplo do complexo de Cartan como a bigraduação

$$
C^{p, q}:=\left(S^{p}\left(g^{*}\right) \otimes A^{q-p}\right)^{G}
$$

com operadores fronteira dados por

$$
d:=1 \otimes d_{A}
$$

e

$$
\delta:=-\mu^{a} \otimes \iota_{a} .
$$

Temos que mostrar que esta construção realmente satisfaz as condições de complexo duplo. Primeiro, a bigraduação é claramente uma bigraduação de 
espaços vetoriais, já que, $S\left(g^{*}\right)$ e $A$ são álgebras. Além disso,

$$
d: C^{p, q}=\left(S^{p}\left(g^{*}\right) \otimes A^{q-p}\right)^{G} \rightarrow\left(S^{p}\left(g^{*}\right) \otimes A^{(q+1)-p}\right)^{G}=C^{p, q+1}
$$

e

$$
\delta: C^{p, q}=\left(S^{p}\left(g^{*}\right) \otimes A^{q-p}\right)^{G} \rightarrow\left(S^{p+1}\left(g^{*}\right) \otimes A^{q-(p+1)}\right)^{G}=C^{p+1, q}
$$

pois $\iota_{a}$ é uma derivação de grau -1 em $A$. Resta mostrar que $d^{2}=0, \delta^{2}=0$ e $d \delta+\delta d=0$. Ora, mas

$$
d_{A}^{2}=0
$$

portanto $d^{2}=0$. Também temos que

$$
\left[\iota_{a}, \iota_{b}\right]=0
$$

assim,

$$
\left(\sum \iota_{a}\right)\left(\sum \iota_{a}\right)=0
$$

$\operatorname{logo} \delta^{2}=0$. E, por fim, como sabemos que $d_{G}^{2}=0$, temos que $(d+\delta)^{2}=0$, $\log O$

$$
d^{2}+\delta^{2}+d \delta+\delta d=0 \quad \Longrightarrow \quad d \delta+\delta d=0
$$

Portanto realmente temos um complexo duplo. Repare que o subespaço $\left(S^{p}\left(g^{*}\right) \otimes A^{m}\right)^{G}$ tem bigraduação $(p, m+p)$ e portanto tem grau total $2 p+m$, o que preserva a graduação original de $\left(S\left(g^{*}\right) \otimes A\right)^{G}$ como uma super álgebra comutativa.

Quando trabalhamos com sequência espectral é importante saber quando ela converge ou não, isto é, saber quando a sequência espectral se estabiliza para algum $r$ :

$$
E_{r}^{p, q}=E_{r+1}^{p, q}=\cdots
$$

Neste sentido, se $A^{k}=0$ para $k<0$, como será nosso caso, temos que a condição D.1.4 é claramente satisfeita e, com isto, a sequência espectral associada ao complexo duplo 8.1.1, 8.1.2 e 8.1.3 irá convergir. Isto será muito importante, já que alguns resultados serão consequência desta convergência.

Provemos agora o seguinte:

Teorema 8.1.1 O termo $E_{1}$ na sequência espectral de 8.1.1, $C^{p, q}$, é

$$
\left(S\left(g^{*}\right) \otimes H(A)\right)^{G}
$$


isto é,

$$
E_{1}^{p, q}=\left(S^{p}\left(g^{*}\right) \otimes H^{q-p}(A)\right)^{G} .
$$

Prova: Como sabemos pela teoria de sequência espectral,

$$
E_{1}^{p, q}=H^{q}\left(C^{p, *}, d\right)
$$

assim, o que temos que fazer é mostrar que

$$
\left(S^{p}\left(g^{*}\right) \otimes H^{q-p}(A)\right)^{G} \cong H^{q}\left(\left(S^{p}\left(g^{*}\right) \otimes A\right)^{G}, 1 \otimes d_{A}\right) .
$$

Ora, mas como $G$ é compacto, dado qualquer $a \in G$ podemos considerar, o mapa

$$
\begin{gathered}
H\left(C_{G}(A), 1 \otimes d_{A}\right) \rightarrow\left(S\left(g^{*}\right) \otimes H(A)\right)^{G} \\
{\left[\int_{G} a(x \otimes \alpha)\right] \mapsto x \otimes[\alpha] .}
\end{gathered}
$$

Como, por definição, $C_{G}(A)$ e $x \in S\left(g^{*}\right)^{G}$ são $G$-invariante, o mapa está bem definido e é injetivo e, como $G$ é compacto, $\forall a \in G$,

$$
\int_{G} a[\alpha] \mu=\int_{G}[\alpha] \mu=[\alpha] \int_{G} \mu=[\alpha]
$$

Logo, fazendo a média, os grupos de cohomologia de $\left(S\left(g^{*}\right) \otimes A, 1 \otimes d_{A}\right)$ são as componentes $G$-invariantes da cohomologia de

$$
\left(S\left(g^{*}\right) \otimes A, 1 \otimes d_{A}\right)
$$

que, por sua vez, são as componentes adequadamente graduadas de $S\left(g^{*}\right) \otimes H(A)$.

Para melhorar o teorema anterior, provemos o seguinte:

Lema 8.1.2 A componente conexa da identidade de $G$ age trivialmente em $H(A)$.

Prova: De fato, lembramos que estamos usando que $H(A)$ herda uma estrutura de $G^{*}$-módulo. Se a pertence a componente conexa da identidade, então $a=\exp t \xi$ para algum $\xi \in g$. Ora, mas por definição

$$
\mathcal{L}_{\xi}=\left.\frac{d}{d t} \rho(\exp t \xi)\right|_{t=0}
$$

desta forma, para mostrar que a componente conexa da identidade de $G$ age trivialmente $H(A)$, basta mostrar que, para todo $\xi \in g, \mathcal{L}_{\xi}$ age trivialmente 
em $H(A)$. Mas sabemos que

$$
\mathcal{L}_{\xi}=\iota_{\xi} d+d \iota_{\xi}
$$

Logo, por definição, $\mathcal{L}_{\xi}$ homotópico em cadeia a 0 em $A$ e temos assim o resultado.

Mostramos então que:

Teorema 8.1.3 Se Gé conexo,

$$
E_{1}^{p, q}=S^{p}\left(g^{*}\right)^{G} \otimes H^{q-p}(A) .
$$

E, assim, pelo corolário D.2.3, temos de imediato que:

Teorema 8.1.4 Se $G$ é conexo e $H^{p}(A)=0$ se $p$ é ímpar, segue que a sequência espectral do complexo de Cartan colapsa no estágio $E_{1}$.

Se $M$ é uma variedade na qual $G$, um grupo de Lie compacto e conexo, age livremente, temos que

$$
H_{G}(\Omega(M))=H^{*}(X)
$$

onde $X=M / G$. Neste caso, o termo $E_{1}$ da sequência espectral é

$$
S\left(g^{*}\right)^{G} \otimes H^{*}(M)
$$

e o termo $E_{\infty}$ é uma versão graduada de $H^{*}(X)$. Repare que pelo teorema anterior, se $H^{p}(\Omega(M))=0$ se $p$ é ímpar, segue que $S\left(g^{*}\right)^{G} \otimes H^{*}(M)$ é a própria versão graduada de $H^{*}(X)$ dada pela sequência espectral.

\section{2}

\section{$H_{G}(A)$ um $S\left(g^{*}\right)^{G}$-módulo e Morfismos de $G^{*}$-módulos}

A partir deste momento, iremos supor que, além de compacto, $G$ também é um grupo de Lie conexo. Considere agora $\phi \in S^{m}\left(g^{*}\right)^{G}$. O operador multiplicação

$$
u \otimes a \mapsto \phi u \otimes a
$$

claramente comuta com $d$ e $\delta$, portanto é um morfismo, sobre si, do duplo complexo $\left(C_{G}(A), d, \delta\right)$ dado por 8.1.1, 8.1.2 e 8.1.3. Além disso, como $\phi \in$ $S^{m}\left(g^{*}\right)^{G}$, temos que este morfismo tem bigrau $(m, m)$, por definição de $C^{p, q}$. Logo, como podemos ver no apêndice, este morfismo induz uma cocadeia

$$
\left(C_{G}(A), d+\delta\right) \rightarrow\left(C_{G}(A), d+\delta\right)
$$


de grau $2 m$ e, portanto um mapa na cohomologia. Mas, por definição, $H_{G}(A)=H\left(C_{G}(A), d+\delta\right)$, portanto o operador multiplicação induz um mapa de $H_{G}(A)$ sobre ele mesmo, ou seja, temos uma estrutura de $S\left(g^{*}\right)^{G}$-módulo para $H_{G}(A)$.

Repare que, como no apêndice, o operador multiplicação também induz mapas no complexo de cocadeia $\left(C^{p, *}, d\right)$ e, consequentemente, em todos $E_{r}$ 'a da sequência espectral. Desta forma, todos os $E_{r}$ 's herdam uma estrutura de $S\left(g^{*}\right)^{G}$-módulo. Observe que por 8.1.4, a estrutura de $S\left(g^{*}\right)^{G}$-módulo herdada é apenas a multiplicação em $S\left(g^{*}\right)^{G}$, isto é,

$$
S\left(g^{*}\right)^{G} \otimes H(A) \rightarrow\left(S\left(g^{*}\right)^{G} \cdot S\left(g^{*}\right)^{G}\right) \otimes H(A) .
$$

Agora, $S\left(g^{*}\right)^{G}$ é finitamente gerado (por [Hel]), portanto, todo ideal de $S\left(g^{*}\right)^{G}$ é finitamente gerado. Logo, $S\left(g^{*}\right)^{G}$ é noetheriano (como em [Chev]). Assim, se $H(A)$ é finitamente gerado, temos por [Chev] que, todos os seus subquocientes, incluindo os $E_{r}$ 's, são finitamente gerados como $S\left(g^{*}\right)^{G}$-módulo. Assim, como por construção, a sequência espectral converge para uma versão graduada de $H_{G}(A)$, provamos que:

Teorema 8.2.1 Se a dimensão de $H(A)$ é finita, então $H_{G}(A)$ é finitamente gerado como um $S\left(g^{*}\right)^{G}$-módulo.

O resultado é imediato, já que, a versão graduada de $H_{G}(A)$ é limite de $S\left(g^{*}\right)^{G}$ módulos finitamente gerados. O que podemos ver também é que:

Teorema 8.2.2 Se a sequência espectral do complexo duplo de Cartan colapsa no estágio $E_{1}$, então $H_{G}(A)$ é um $S\left(g^{*}\right)^{G}$-módulo livre.

Prova: Como a sequência espectral colapsa em $E_{1}$, temos que $E_{1} \cong g r H_{G}(A)$, a versão graduada de $H_{G}(A)$. Ora, mas pelo teorema D.2.2, o isomorfismo $E_{1} \cong \operatorname{gr} H_{G}(A)$ é um isomorfismo do mapa graduado na cohomologia que preserva a estrutura de módulo, portanto um isomorfismo de $S\left(g^{*}\right)^{G}$-módulos. Assim como, por 8.1.4, $E_{1}$ é claramente um $S\left(g^{*}\right)^{G}$-módulo livre, pelo produto no primeiro fator, $\operatorname{gr}_{G}(A)$ é um $S\left(g^{*}\right)^{G}$-módulo livre e portanto, $H_{G}(A)$ é um $S\left(g^{*}\right)^{G}$-módulo livre.

$\mathrm{Na}$ verdade, pelas considerações feitas aqui e por 8.1.4, como a sequência espectral colapsa no nível $E_{1}$, temos a equivalência

$$
H_{G}(A) \cong H(A) \otimes S\left(g^{*}\right)^{G}
$$


como $S\left(g^{*}\right)^{G}$-módulo. Desta forma, tensorizando sobre $S\left(g^{*}\right)^{G}$ cada lado desta equivalência por $\mathbb{C}$, temos que

$$
H_{G}(A) \otimes_{S\left(g^{*}\right)^{G}} \mathbb{C} \cong H(A) \otimes S\left(g^{*}\right)^{G} \otimes_{S\left(g^{*}\right)^{G}} \mathbb{C} \cong H(A) \otimes \mathbb{C} \cong H(A)
$$

pelas propriedades de produto tensorial. Desta forma, se $A=\Omega(M)$, podemos calcular a cohomologia de de Rham de $M$ em termos de sua cohomologia equivariante.

Considere agora $A$ e $B G^{*}$-módulos e $\rho: A \rightarrow B$ um morfismo de grau 0. Ele induz um mapa $\rho^{*}$ em cohomologia. Consideremos a notação que usamos no apêndice. Como $G$ é conexo temos que $\left(S^{p}\left(g^{*}\right) \otimes H^{q-p}(A)\right)^{G} \cong$ $S^{p}\left(g^{*}\right)^{G} \otimes H^{q-p}(A)$. Portanto,

$$
\begin{array}{ccc}
E_{1}^{p, q}(A) & = & S^{p}\left(g^{*}\right)^{G} \otimes H^{q-p}(A) \\
\rho_{1} \downarrow & & \downarrow 1 \otimes \rho^{*} \\
E_{1}^{p, q}(B) & = & S^{p}\left(g^{*}\right)^{G} \otimes H^{q-p}(B) .
\end{array}
$$

Assim, se $\rho^{*}$ é um isomorfismo, $\rho_{1}$ é um isomorfismo. Como consequência, $\rho_{r}$ é um isomorfismo. Segue que, como $\lim \rho_{r}=g r \rho_{\star}$, onde

$$
\rho_{\star}: H_{G}(A) \rightarrow H_{G}(B)
$$

é o mapa induzido por $\rho$, então, temos que $g r \rho_{\star}$ é um isomorfismo. Logo $\rho_{\star}$ é um isomorfismo. Com isto provamos, graças aos teoremas D.2.2 e 8.1.3, o seguinte:

Teorema 8.2.3 Se o mapa induzido $\rho^{*}$ na cohomologia standard é um isomorfismo, então o mesmo ocorre com o mapa induzido por $\rho_{\star}$.

Em alguns casos pode ser mais fácil calcular a cohomologia equivariante do que a cohomologia standard, neste caso, o teorema anterior pode facilitar distinguir quando, por exemplo, certas variedades não têm a mesma cohomologia, já que, se mostrarmos que duas variedades tem cohomologia equivariantes diferentes, elas terão cohomologia de de Rham diferentes.

Um exemplo de seu uso pode ser calcular a cohomologia de um espaço homogêneo $G / K$. A cohomologia de de Rham, neste caso, pode ser um pouco complicada de se trabalhar, entretanto, como vimos no capítulo anterior, temos uma fórmula para o cálculo de sua cohomologia equivariante. 


\section{3 \\ Restrição de Grupo}

Nesta seção faremos construções que são relativamente imediatas dados os resultados que já temos até aqui, todavia, são construções importantes que servem como base para o resultado que desejamos neste trabalho.

Para começar, novamente, temos $G$ um grupo de Lie compacto e conexo. Consideremos, agora, $K$ um subgrupo fechado de $G$. Lembremos que $K$ fechado implica $K$ um subgrupo de Lie de $G$ e que $G / K$ está bem definido como uma variedade.

Agora, como $K$ é um subgrupo de Lie de $G$, temos que $k$, a álgebra de Lie de $K$, é uma subálgebra de Lie de $g$. Assim, também temos a injetividade de álgebras de Lie

$$
k \rightarrow g
$$

E ainda, $k$ como uma subálgebra de Lie de $g$ é, em particular, um subespaço vetorial de $g$. Desta forma, podemos estender uma base de $k$ à uma base de $g$ e, naturalmente, também temos a injetividade das super álgebras de Lie

$$
\bar{k} \rightarrow \bar{g}
$$

de forma que, cada $G^{*}$-módulo se torna um $K^{*}$-módulo pela restrição. Nada muito diferente do que fizemos na seção de cohomologia equivariante de espaços homogêneos do capítulo anterior.

Agora, o mapa injetivo $k \rightarrow g$ induz um mapa sobrejetivo no dual, isto é, induz uma projeção

$$
g^{*} \rightarrow k^{*}
$$

que se estende, de forma a preservar o produto, a um mapa

$$
S\left(g^{*}\right) \rightarrow S\left(k^{*}\right)
$$

Consideremos então $A$ um $G^{*}$-módulo, pela restrição temos que $A$ é um $K^{*}$-módulo. Então, pelas construções feitas, e a projeção natural de $A$ como $G^{*}$ módulo em $A$ como $K^{*}$-módulo, preservando a invariância, nos dá o morfismo

$$
\left(S\left(g^{*}\right) \otimes A\right)^{G} \rightarrow\left(S\left(k^{*}\right) \otimes A\right)^{K} .
$$

E, como as derivações do domínio agem diretamente ou pela restrição 
como as derivações do contradomínio do mapa, claramente o morfismo se entrelaça, no sentido de comutar, com $\delta$ e $d$ e, portanto, representa um morfismo de bicomplexo de bigrau $(0,0)$. Ou seja, representa um morfismo

$$
C_{G}(A) \rightarrow C_{K}(A)
$$

Assim, temos o mapa restrição induzido na cohomologia

$$
H_{G}(A) \rightarrow H_{K}(A)
$$

e em cada estágio da sequência espectral por D.2.1 e D.2.2.

Por hipótese $G$ é conexo então, pelo lema 8.1.2, $G$ age trivialmente em $H(A)$. Mas $K$ é subgrupo de $G$, portanto $K$ também age trivialmente em $H(A)$. Desta forma, considerando a sequência espectral do complexo de Cartan $C_{K}(A)$, também temos que

$$
E_{1}^{p, q}(K)=S^{p}\left(k^{*}\right)^{K} \otimes H^{q-p}(A)
$$

Assim, o morfismo restrição induzido por $C_{G}(A) \rightarrow C_{K}(A)$ no nível $E_{1}$ da sequência espectral é

$$
S\left(g^{*}\right)^{G} \otimes H(A) \rightarrow S\left(k^{*}\right)^{K} \otimes H(A)
$$

ou seja, neste caso o morfismo restrição é a restrição aplicada aos fatores a esquerda do produto tensorial. Desta forma, temos o seguinte:

Teorema 8.3.1 Se o mapa

$$
S\left(g^{*}\right)^{G} \rightarrow S\left(k^{*}\right)^{K}
$$

é um isomorfismo, então o mapa restrição

$$
H_{G}(A) \rightarrow H_{K}(A)
$$

na cohomologia equivariante é um isomorfismo.

Prova: De fato, se $S\left(g^{*}\right)^{G} \rightarrow S\left(k^{*}\right)^{K}$ é um isomorfismo temos, pelo feito acima, um isomorfismo no nível $E_{1}$ na sequência espectral, logo um isomorfismo em todos os níveis da sequência espectral. Assim, pelo teorema D.2.2, isomorfismo na cohomologia equivariante. 
Um exemplo que satisfaz nossas hipóteses é justamente o toro maximal como veremos na próxima seção.

\section{4}

\section{Cohomologia do Fibrado Flag Homogêneo}

Iremos, finalmente, nesta seção, exibir a fórmula para cacular a cohomologia de um fibrado flag homogêneo, o resultado que buscávamos neste trabalho. Então para começar a seção é interessante relembrar algumas definições que fizemos no capítulo 2.

Primeiro, um $G$ fibrado principal é um fibrado com fibra e grupo estrutural $G$. Agora, considere $\pi: M \rightarrow X$ um $G$ fibrado principal e que $G$ age em uma variedade $F$. Consideramos o fibrado associado como sendo $[\pi]: P=(M \times F) / G \rightarrow X$. Repare que este fibrado tem fibra $F$. Suponhamos agora que $H$ é um subgrupo fechado de $G$. Temos uma ação natural de $G$ em $G / H$ pela multiplicação. Desta forma, definimos o fibrado associado homogêneo como o fibrado associado com fibra $G / H$. Vale ressaltar que $H$ age em $M$ pela restrição. Então podemos identificar o espaço das órbitas desta ação, $M / H$ com o fibrado associado $[\pi]: P=(M \times G / H) / G \rightarrow X$, lembrado que $M / H$ é uma variedade, já que, $H$ é um subgrupo de Lie compacto.

Além disso, temos uma identificação natural de $X$ e $M / G$. Assim, considerando simplesmente $\pi: M \rightarrow M / G$ como o fibrado principal, identificamos o fibrado associado homogêneo pelo diagrama

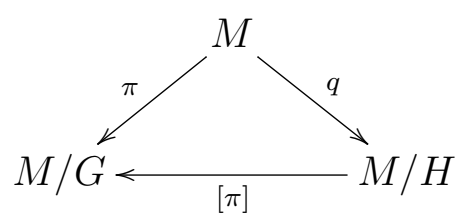

onde o mapa $[\pi]$ projeta a órbita de $H$ em $M$ na órbita de $G$ em $M$ como uma submersão.

Por fim, considerando $\rho: M \rightarrow M / G$ o fibrado principal, um fibrado flag homogêneo é um fibrado homogêneo onde $H=T$ e $T$ é o toro maximal, isto é, um subgrupo compacto, conexo e abeliano de $G$ que é maximal em relação a inclusão. Repare que, como $G$ é compacto e conexo, $T$ é um subgrupo de 
Cartan de $G$. Desta forma, temos o diagrama

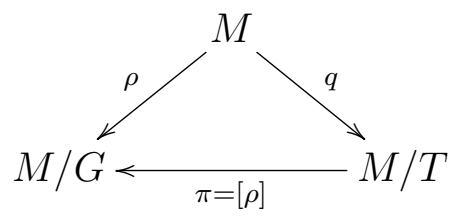

onde $\pi$ projeta a órbita de $T$ em $M$ na órbita de $G$ em $M$ como uma submersão.

O mapa $\pi$ do diagrama acima vem a ser muito importante no nosso trabalho, já que, basicamente, queremos calcular a cohomologia do fibrado flag associado, ou simplesmente $M / T$, em relação a base $M / G$. Para fazer isto, queremos, de alguma forma, usar o teorema 8.3.1, vejamos então as propriedades de $T$, o toro maximal, que torna isto possível.

Consideremos $K$ o subgrupo de Lie de $G$ normalizador de $T$, isto é,

$$
K=:\left\{a \in G \mid a T a^{-1} \subset T\right\} .
$$

Uma forma equivalente de definir $K$ é

$$
K=\{a \in G \mid \operatorname{Ad}(a) t \subset t\}
$$

pois, por um lado, como sabemos, $\exp (s A d(a) \xi)=a \exp (s \xi) a^{-1} \in T$ para todo $a \in G, \xi \in t$ e $s \in \mathbb{R}$. Para ver a volta da equivalência, se $A d(a) t \subset t$, temos que $A d(a) \xi \in t$ para todo $\xi \in t$, então, considerando $x \in T$ tal que $(\exp \xi)=x$, temos que $a x a^{-1}=\exp (A d(a) \xi) \in T$.

Defina agora

$$
\mathcal{W}=: K / T
$$

o grupo de Weyl de $G$. Afirmamos que a subálgebra de Lie de $K$ é $t$. De fato, se $k$ é a álgebra de Lie de $K$, então $t \subset k$. Além disso, para todo $s \in \mathbb{R}$, de $\xi \in k, \exp (s \xi) \in K$. Ou seja, temos que $\exp (\operatorname{sad}(\xi)) t=A d(\exp (s \xi)) t \subset t$. Assim, dado $\nu \in g$, derivando temos

$$
[\xi, \nu]=\left.\frac{d}{d s} \exp (\operatorname{sad}(\xi)) \nu\right|_{s=0} \in t
$$

Logo, como $t$ é uma álgebra de Cartan, $\xi \in t$, logo $k \subset t$. Vale notar também que $\mathcal{W}$ mede o número de componentes conexas de $K$, isto é, a cardinalidade de $\mathcal{W}$ é o número de componentes conexas de $K$, portanto $\mathcal{W}$ é um grupo finito. 
Assim, com as informações acima, temos que

$$
S\left(k^{*}\right)^{K}=S\left(t^{*}\right)^{K}
$$

Além disso, como $T$ por hipótese é abeliano, a ação adjunta é trivial, isto é, pela própria definição da ação adjunta, como $T$ é abeliano, $T$ age trivialmente em $t$, e pelo contragradiente da representação adjunta, trivialmente em $t^{*}$, portanto em $S\left(t^{*}\right)$. Desta forma, como $T \subset K$ age trivialmente em $S\left(t^{*}\right)$, temos que

$$
S\left(k^{*}\right)^{K}=S\left(t^{*}\right)^{K}=S\left(t^{*}\right)^{\mathcal{W}} .
$$

A construção acima nos permite aplicar o teorema 8.3.1, já que, por [Hel], o teorema de Chevalley garante que o mapa restrição

$$
S\left(g^{*}\right)^{G} \rightarrow S\left(t^{*}\right)^{\mathcal{W}}
$$

é um isomorfismo e, portanto,

$$
H_{G}(A) \rightarrow H_{K}(A)
$$

é um isomorfismo.

Agora, pelo que fizemos na seção anterior e acima, a inclusão $T \rightarrow K$ induz um morfismo de complexos duplos

$$
C_{K}(A) \rightarrow C_{T}(A)^{\mathcal{W}}
$$

por 8.4.1. Isto induz o mapa em cohomologia

$$
H_{K}(A) \rightarrow H_{T}(A)^{\mathcal{W}}
$$

e em cada nível da sequência espectral. Em particular, como $K \subset G$, a equação 8.1.4 é válida, temos um morfismo no nível $E_{1}$

$$
S\left(k^{*}\right)^{K} \otimes H(A) \rightarrow\left(S\left(t^{*}\right)^{\mathcal{W}} \otimes H(A)\right)^{T} \cong S\left(t^{*}\right)^{\mathcal{W}} \otimes H(A) .
$$

Contudo, como $S\left(k^{*}\right)^{K}=S\left(t^{*}\right)^{\mathcal{W}}$, o morfismo acima é o morfismo identidade, portanto, aplicando o teorema D.2.2,

$$
H_{K}(A)=H_{T}(A)^{\mathcal{W}}
$$

Provamos, assim, o seguinte teorema: 
Teorema 8.4.1 Seja $G$ um grupo de Lie compacto e conexo, $T$ um toro maximal e $\mathcal{W}$ sua álgebra de Weil, então para qualquer $G^{*}$-módulo A temos que

$$
H_{G}(A) \cong H_{T}(A)^{\mathcal{W}}
$$

Iremos mostrar agora que:

\section{Teorema 8.4.2}

$$
H_{G}(A) \otimes_{S\left(t^{*}\right)^{\mathcal{w}}} S\left(t^{*}\right) \cong H_{T}(A) .
$$

Prova: Para ver isto temos que observar primeiro que o produto tensorial

$$
C_{K}(A) \otimes_{S\left(t^{*}\right)^{\mathcal{W}}} S\left(t^{*}\right)
$$

é um bicomplexo. Como é um produto tensorial sobre $S\left(t^{*}\right)^{\mathcal{W}}$ esperamos que dados $\phi \in S\left(t^{*}\right)^{\mathcal{W}}, \alpha \in C_{K}(A)$ e $\beta \in S\left(t^{*}\right)$ tenhamos que $\alpha \otimes \phi \beta=\phi \alpha \otimes \beta$, mas isto está bem definido pela multiplicação em $S\left(t^{*}\right)$ e por $C_{K}(A)$ ser um $S\left(t^{*}\right)^{\mathcal{W}}$-módulo. Inclusive, o fato de $C_{K}(A)$ ser um $S\left(t^{*}\right)^{\mathcal{W}}$-módulo garante que $\phi$ comute com o operdador fronteira de $C_{K}(A)$, assim, como também comuta com o operador fronteira de $S\left(t^{*}\right)$, temos que $C_{K}(A) \otimes_{S\left(t^{*}\right)^{\mathcal{W}}} S\left(t^{*}\right)$ é realmente um bicomplexo.

Além disso, temos um morfismo canônico de bicomplexos dado por

$$
\begin{array}{ccc}
C_{K}(A) \otimes_{S\left(t^{*}\right)^{\mathcal{W}} S\left(t^{*}\right)} & \rightarrow & C_{T}(A) \\
\alpha \otimes \beta & \mapsto & \beta \alpha
\end{array}
$$

já que, $C_{T}(A)=\left(S\left(t^{*}\right) \otimes A\right)^{T}$. O que induz morfismos em cada nível da sequência espectral. Em particular, no primero nível da sequência espectral, estão bem definidos $C_{K}(A)$ e $C_{T}(A)$. Assim, lembrando que $S\left(k^{*}\right)^{K}=S\left(t^{*}\right)^{\mathcal{W}}$, o morfismo de bicomplexo comentado acima no nível $E_{1}$ pode ser escrito como:

$$
\begin{array}{ccc}
S\left(t^{*}\right)^{\mathcal{W}} \otimes H(A) \otimes_{S\left(t^{*}\right)^{\mathcal{W}} S\left(t^{*}\right)} & \rightarrow & S\left(t^{*}\right) \otimes H(A) \\
\phi \otimes \alpha \otimes \beta & \mapsto & \beta \phi \otimes \alpha .
\end{array}
$$

Ora, mas o produto tensorial $S\left(t^{*}\right)^{\mathcal{W}} \otimes H(A)$ é sobre os complexos e temos que

$$
S\left(t^{*}\right)^{\mathcal{W}} \otimes H(A) \cong H(A) \otimes S\left(t^{*}\right)^{\mathcal{W}}
$$

e, portanto,

$$
S\left(t^{*}\right)^{\mathcal{W}} \otimes H(A) \otimes_{S\left(t^{*}\right)^{\mathcal{W}}} S\left(t^{*}\right) \cong H(A) \otimes\left(S\left(t^{*}\right)^{\mathcal{W}} \otimes_{S\left(t^{*}\right)^{\mathcal{W}}} S\left(t^{*}\right)\right)
$$

Assim, por propriedades de produto tensorial, temos que

$$
S\left(t^{*}\right)^{\mathcal{W}} \otimes H(A) \otimes_{S\left(t^{*}\right)^{\mathcal{W}}} S\left(t^{*}\right) \cong S\left(t^{*}\right) \otimes H(A) .
$$


Segue que o morfismo no nível $E_{1}$ é um isomorfismo e, portanto, pelo teorema D.2.2, um isomorfismo em todos os níveis. Logo,

$$
H_{K}(A) \otimes_{S\left(t^{*}\right)^{\mathcal{W}}} S\left(t^{*}\right) \cong H_{T}(A) .
$$

E, como $H_{K}(A) \cong H_{G}(A)$,

$$
H_{G}(A) \otimes_{S\left(t^{*}\right)^{\mathcal{W}}} S\left(t^{*}\right) \cong H_{T}(A)
$$

como queriamos mostrar.

O resultado central desta dissertação vai ser uma tradução dos teoremas 8.4.1 e 8.4.2, que são de natureza puramente algébricos, ao ámbito geométrico. Para fazer isto, iremos usar fortemente os homomorfismos característicos referentes a cada grupo de Lie, $G$ e $T$.

Para fazer a passagem da álgebra para a geometria, consideremos $M$ uma variedade em que $G$ age livremente. Defina:

$$
\begin{aligned}
& X:=M / G, \\
& Y:=M / T \\
& \pi: Y \rightarrow X
\end{aligned}
$$

o mapa que leva a $T$ órbita na $G$ órbita. Repare que, pelo que fizemos no início da seção, $M / T$ representa o fibrado associado. Desta forma estamos nas condições iniciais do problema central desta dissertação. Repare ainda que o mapa $\pi$ induz o mapa

$$
\pi^{*}: H^{*}(X) \rightarrow H^{*}(Y)
$$

na cohomologia. Ora, mas pelo teorema 8.4.1 aplicado a $A=\Omega(M)$, podemos provar que:

Teorema 8.4.3 O mapa

$$
\pi^{*}: H^{*}(X) \rightarrow H^{*}(Y)^{\mathcal{W}}
$$

é um isomorfismo.

Prova: De fato, como $G$ e $T$ (pela restrição) agem livremente em $M$, temos $\Omega(M)$ satisfaz a condição $(\mathbf{C})$ para ambos os casos e o pullback pela projeção 
de $\Omega(X)$ e $\Omega(Y)$ representam as formas básicas em cada um dos casos, portanto

$$
H_{G}(\Omega(M))=H_{\text {bas }}(\Omega(M) \otimes W) \cong H(\Omega(X), d)=H^{*}(X)
$$

e

$$
H_{T}(\Omega(M)) \cong H(\Omega(Y), d)=H^{*}(Y) .
$$

Assim, pelos isomorfismos acima, o mapa $H_{G}(\Omega(M)) \rightarrow H_{T}(\Omega(M))^{\mathcal{W}}$ induzido pela restrição de grupo é o mapa $\pi^{*}$, isto é, pelo teorema 8.4.1,

$$
\pi^{*}: H^{*}(X) \rightarrow H^{*}(Y)^{\mathcal{W}}
$$

é um isomorfismo.

Desta forma temos uma tradução completa para o ámbito geométrico do teorema 8.4.1, que inclui os grupos e o morfismo entre eles.

O teorema acima é forte, iremos aprimorar (e com isto finalizar o resultado principal desta dissertação) considerando os geradores de $S\left(t^{*}\right)^{\mathcal{W}}$ e a equação 8.4.4 em uma perspectiva geométrica. Então, antes de provar o teorema 3.1.3, lembremos o que ele afirma.

Com a notação usual, o teorema afirma que a cohomologia do fibrado associado $Y$ é o quociente

$$
H^{*}(X)\left[\sigma^{1}, \cdots, \sigma^{r}\right] / I
$$

onde $I$ é um ideal construído, não diretamente, a partir da base de $t^{*}$. É claro que até agora não sabemos o que é o ideal $I$ ou as variáveis $\left(\sigma^{i}\right)^{\prime}$ 's. Entretanto, provar o teorema, neste momento, é basicamente expressar cuidadosamente cada um destes termos para aprimorar o teorema 8.4.3 como veremos.

Primeiro, graças também a [Hel] na prova do teorema de Chevalley, como o grupo de Weyl, $\mathcal{W}$, é finito, os polinômios invariantes sobre $\mathcal{W}$ tem geradores algébricamente independentes finitos, isto é, pelo teorema de Chevalley, $S\left(t^{*}\right)^{\mathcal{W}}$ com sua representação polinomial é gerado finitamente. Consideremos então $x_{1}, \cdots, x_{r}$ uma base de $t^{*} \mathrm{e}$

$$
p_{i}\left(x_{1}, \cdots, x_{r}\right)
$$

com $i=1, \cdots, n$, geradores de $S\left(t^{*}\right)^{\mathcal{W}}$. 
Na seção 7.4 fizemos uma descrição muito precisa do mapa de ChernWeil, no caso do toro maximal, podemos vê-lo através do mapa

$$
\kappa_{T}: S\left(t^{*}\right)^{T} \rightarrow H_{T}(\Omega(M)) \cong H^{*}(Y)
$$

que leva uma base $x_{1}, \cdots x_{r}$ nas classes características correspondentes $\sigma^{1}, \cdots, \sigma^{r}$ em $H^{*}(Y)$. Então aqui surgem os $\left(\sigma^{j}\right)^{\prime}$ s. Repare que, como $T$ é abeliano, sua ação em $S\left(t^{*}\right)$ é trivial, portanto $S\left(t^{*}\right)^{T}=S\left(t^{*}\right)$, nos permitindo escrever o mapa acima esquecendo a invariância em $T$. Além disso, a invariância sobre $\mathcal{W}$ é mantida pelo mapa, isto é, elementos invariantes sobre $\mathcal{W}$ são levados em elementos invariantes sobre $\mathcal{W}$.

Por outro lado, também temos o mapa de Chern-Weil para $G$

$$
\kappa_{G}: S\left(g^{*}\right)^{G} \rightarrow H_{G}(\Omega(M)) \cong H^{*}(X) .
$$

Mas repare que, pelo teorema de Chevalley, o mapa restrição

$$
S\left(g^{*}\right)^{G} \rightarrow S\left(t^{*}\right)^{\mathcal{W}}
$$

é um isomorfismo, ou seja, o homomorfismo característico $\kappa_{G}$ mapeia $p_{1}, \cdots, p_{k}$ nas classes características correspondentes $c_{1}, \cdots, c_{k}$ em $H^{*}(X)$.

Assim, como o mapa restrição, pelo teorema de Chevalley, é um isomorfismo, passando pelo mapa $\pi^{*}$ como vimos antes, temos que o diagrama

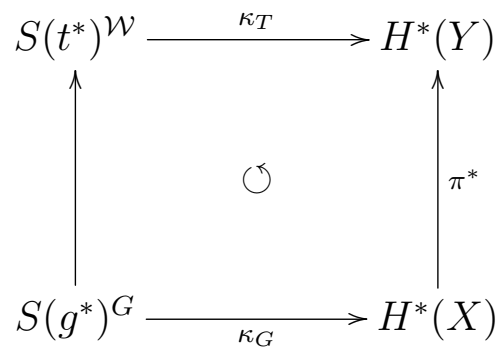

é comutativo.

Agora, pelo teorema 8.4.2 e a tradução geométrica que vem do teorema 8.4.3, temos o isomorfismo

$$
\begin{array}{clc}
H^{*}(X) \otimes_{S\left(t^{*}\right)^{\mathcal{W}} S\left(t^{*}\right)} & \rightarrow & H^{*}(Y) \\
a \otimes \phi & \mapsto & \pi^{*}(a) \kappa_{T}(\phi) .
\end{array}
$$

O mapa acima está bem definido, já que podemos ver $H^{*}(X)$ como um 
$S\left(t^{*}\right)^{\mathcal{W}}$-módulo pelo isomorfismo no teorema de Chevalley. Desta forma, a multiplicação de um polinômio $\mathcal{W}$ invariante é dado por sua classe característica em $H^{*}(Y)$ através do mapa $\kappa_{T}$.

Então, nosso objetivo é relacionar a equação acima com $H^{*}(X)\left[\sigma^{1}, \cdots, \sigma^{r}\right]$ afim de provar o teorema 3.1.3.

Ora, mas um polinômio $p \in S\left(t^{*}\right)^{\mathcal{W}}$ corresponde a um polinômio $P \in$ $S\left(g^{*}\right)^{G}$ pelo isomorfismo. Desta forma, ao multiplicar $p$ em $H^{*}(X)$ o vemos através do produto da classe característica por $G$ de $P$, isto é, multiplicamos em $H^{*}(X)$ por $\kappa_{G}(P)$.

Consideremos, então, um mapa natural dado por

$$
\begin{array}{ccc}
H^{*}(X) \otimes_{S\left(t^{*}\right)^{\mathcal{W}} S\left(t^{*}\right)} & \rightarrow & H^{*}(X)\left[x_{1}, \cdots, x_{r}\right] / J \\
a \otimes \phi & \mapsto & {[a \cdot \phi]}
\end{array}
$$

onde $J$ é um ideal. Para compreender exatamente como é este ideal, lembremos que $S\left(t^{*}\right)^{\mathcal{W}}$ é gerado pelos polinômios $p_{1}, \cdots, p_{n}$, portanto gerado finitamente. Cada $p_{j}$ tem uma correspondência $P_{j}$ nos polinômios $G$-invariantes em $g$, isto é, em $S\left(g^{*}\right)^{G}$. Assim, definimos $J$ como sendo o ideal gerado por $p_{1}-\kappa_{G}\left(P_{1}\right), \cdots, p_{n}-\kappa_{G}\left(P_{n}\right)$.

O mapa foi dito natural pois estamos considerando que ele mapeia $a \otimes \phi$ em $[a \cdot \phi]$, onde $a \cdot \phi$ representa um polinômio sobre $H^{*}(X)$ com variáveis em $t^{*}$. Repare que o mapa está bem definido, já que, se $\alpha \in S\left(t^{*}\right)^{\mathcal{W}}, \alpha$ é gerado pelos $p_{j}$ 's e está na imagem pelo homomorfismo característico e portanto

$$
[\alpha \cdot 1]=[1 \cdot \alpha]=[0]
$$

isto é, o ideal $J$ permite que o mapa respeite a estrutura do produto tensorial sobre $S\left(t^{*}\right)^{\mathcal{W}}$.

Além disso, não é muito difícil ver que o mapa é um isomorfismo, a sobrejetividade é imediata. Quanto a injetividade, se $a_{1} \otimes \phi_{1}, a_{2} \otimes \phi_{2} \in$ $H^{*}(X) \otimes_{S\left(t^{*}\right) \mathcal{W}} S\left(t^{*}\right)$ e $\left[a_{1} \cdot \phi_{1}\right]=\left[a_{2} \cdot \phi_{2}\right]$, temos, por construção, que $a_{1} \cdot \phi_{1} \mathrm{e}$ $a_{2} \cdot \phi_{2}$ diferem por um termo no ideal $J$ e, portanto, existe $\alpha \neq 0 \in S\left(t^{*}\right)^{\mathcal{W}}$ tal que, considerando $\bar{\alpha}$ como sendo o elemento correspondente a $\alpha$ em $S\left(g^{*}\right)^{G}$, $\left(\alpha-\kappa_{G}(\bar{\alpha})\right)+a_{1} \cdot \phi_{1}=a_{2} \cdot \phi_{2}$ e, portanto, $(1 \otimes \alpha-\alpha \otimes 1)+a_{1} \otimes \phi_{1}=a_{2} \otimes \phi_{2} \mathrm{e}$, assim, $a_{1} \otimes \phi_{1}=a_{2} \otimes \phi_{2}$. Além disso, o mapa é claramente linear e respeita a estrutura de produto logo, é um isomorfismo de álgebras. Repare que o mapa 
inverso é dado por $[a \cdot \phi] \mapsto a \otimes \phi$.

Temos que traduzir a composição dos isomorfismos

$$
H^{*}(X)\left[x_{1}, \cdots, x_{r}\right] / J \rightarrow H^{*}(X) \otimes_{S\left(t^{*}\right)^{\mathcal{W}}} S\left(t^{*}\right)
$$

e

$$
H^{*}(X) \otimes_{S\left(t^{*}\right)^{\mathcal{W}}} S\left(t^{*}\right) \rightarrow H^{*}(Y) .
$$

Ora, mas pelas definições dos mapas, o mapa composição

$$
H^{*}(X)\left[x_{1}, \cdots, x_{r}\right] / J \rightarrow H^{*}(Y)
$$

envia $\left[a_{1} x_{1}+\cdots+a_{r} x_{r}\right]$ em $\pi^{*}\left(a_{1}\right) \sigma^{1}+\cdots+\pi^{*}\left(a_{r}\right) \sigma^{r}$, desta forma, pelo mapa, temos a identificação natural

$$
H^{*}(X)\left[x_{1}, \cdots, x_{r}\right] / J \cong H^{*}(X)\left[\sigma^{1}, \cdots, \sigma^{r}\right] / I
$$

onde $I$ é o ideal gerado por $\kappa_{T}\left(p_{1}\right)-\pi^{*} \kappa_{G}\left(P_{1}\right), \cdots, \kappa_{T}\left(p_{n}\right)-\pi^{*} \kappa_{G}\left(P_{n}\right)$.

Logo, como a classe característica de $p_{j}$ por $\kappa_{G}$ é $c_{j}$, provamos que

$$
H^{*}(Y) \cong H^{*}(X)\left[\sigma^{1}, \cdots, \sigma^{r}\right] /\left(<\pi^{*} c_{i}-p_{i}\left(\sigma^{1}, \cdots, \sigma^{r}\right) \mid i=1, \cdots n>\right) .
$$

Por fim, provamos então o teorema 3.1 .3 e exibimos uma fórmula explícita para calcular o anel de cohomologia de um fibrado flag homogêneo através de um quociente de um anel polinomial com coeficientes no anel de cohomologia da base.

Vejamos então alguns exemplos.

Exemplo 8.4.4 (Cohomologia do Fibrado Flag $G / T$ ) Repare que

$$
G / T \cong(G \times(G / T)) / G
$$

Neste caso, o diagrama que representa o fibrado flag homogêneo pode ser visto como

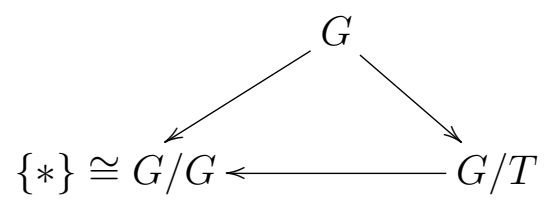


e, portanto, pelo teorema 3.1.3,

$$
H^{*}(G / T)=H^{*}(*)\left[x_{1}, \cdots, x_{n}\right] / J .
$$

Mas, como sabemos, $H^{*}(*)=H^{0}(*)=\mathbb{R}$, ou para ser mais preciso, como estamos trabalhando em $\mathbb{C}, H^{*}(*)=\mathbb{C}$. Além disso, os polinômios $p_{1}, \cdots, p_{n}$ geradores de $S\left(t^{*}\right)^{W}$, que podemos escolher de forma a serem homogêneos, não são constantes e, por isto, tem grau estritamente positivo. Sendo mais exato, ainda pelo teorema de Chevalley, os polinômios geradores são polinômios de grau maior ou igual que um mais a identidade 1.

Entretanto, estamos trabalhando com espaços conexos, desta forma, a cohomologia $H_{G}^{0}$ é canonicamente isomorfa a $\mathbb{C}$ e a ação de $\mathbb{C}$ é dada com base na estrutura de $\mathbb{C}$-espaço vetorial correspondente a ação de $H_{G}^{0}$ dada pela estrutura de álgebra. Em suma, o que estamos querendo dizer é que, $1 \in S\left(t^{*}\right)^{W}$ representa o próprio $1 \in S\left(g^{*}\right)^{G}$ pelo isomomorfismo de Chevalley que, por sua vez, é enviado em $1 \in \mathbb{C} \cong H_{G}^{0}$ pelo homomorfismo característico $\kappa_{G}$, ou seja, $1-\kappa_{G}(1)=0$ e a relação que vem da identidade, assim como de qualquer polinômio constante, é trivial. Assim, como estamos interessados nesta relação, estamos apenas considerando os geradores de grau maior que 0 .

Ora, mas o grau de um elemento em $S\left(g^{*}\right)$ é considerado o dobro de seu grau, portanto $\kappa_{G}\left(P_{j}\right)$ tem grau maior ou igual a 2 . Mas a cohomologia de $H^{k}(*)$ é 0 para grau maior que 0 , e, portanto, $\kappa_{G}\left(P_{j}\right)=0$.

Logo, $p_{j}-\kappa_{G}\left(P_{j}\right)=p_{j}$. Segue então que

$$
H(G / T) \cong \mathbb{C}\left[x_{1}, \cdots, x_{r}\right] / J
$$

onde $J$ é simplesmente o ideal gerado pelos polinômios invariantes que zeram na origem. Repare ainda que, como sabemos, $\mathbb{C}\left[x_{1}, \cdots, x_{r}\right]=S\left(t^{*}\right)$.

Exemplo 8.4.5 (Cohomologia do Fibrado Flag $S O(3) / \mathbb{S}^{1}$ ) $O$ que iremos fazer é basicamente uma aplicação do exemplo anterior. Estamos afirmando neste caso que $\mathbb{S}^{1}$ é um toro maximal de $S O(3)$. Na verdade vale comentar que um toro é um produto finito de cópias de $\mathbb{S}^{1}$, então um toro em um grupo de Lie é um mergulho deste produto de cópias no grupo. Então, o que estamos afirmando sem provar é que $\mathbb{S}^{1}$ mergulhado em $S O(3)$ é um toro maximal. Além disso,

$$
S O(3) / \mathbb{S}^{1} \cong \mathbb{S}^{2}
$$


Em outras palavras, iremos recuperar a cohomologia da esfera bidimensional. Repare primeiro que $\mathbb{S}^{1}$ tem dimensão 1 e portanto $S\left(\left(\operatorname{Lie}\left(\mathbb{S}^{1}\right)\right)^{*}\right) \cong \mathbb{C}[x]$, o anel polinomial em uma variável. Outro ponto é que a álgebra de Lie de $S O(3)$ é a mesma que a de $S U(2)$ e $S U(2)$ é difeomorfo a esfera $\mathbb{S}^{3}$. Mas $\mathbb{S}^{1}$ divide $\mathbb{S}^{3}$ em duas componentes conexas idênticas, desta forma temos que o normalizador $\mathbb{S}^{1}$ está em duas componentes conexas distintas. Podemos ver então que o grupo de Weil, que mede o número de componentes conexas do normalizador, é $\mathbb{Z}^{2}$.

$$
\begin{aligned}
\text { Assim, } \mathbb{C}[x]^{\mathbb{Z}^{2}}= & \mathbb{C}\left[x^{2}\right] e \\
& H^{*}\left(\mathbb{S}^{2}\right) \cong \mathbb{C}[x] /<x^{2}>=\mathbb{C}+\mathbb{C} x .
\end{aligned}
$$

O primeiro termo da soma representa a cohomologia de grau 0 e o segundo de grau 2 .

\section{Exemplo 8.4.6 (FIbrado Flag Homogêneo com Cohomologia Produto)}

Suponha que G seja um grupo de Lie semi-simples, isto é, sua álgebra de Lie é uma soma direta de álgebras de Lie simples (seus ideais são triviais) ou, simplesmente, sua álgebra de Lie não contém ideais solúveis além de 0. Isso implica que os polinômios homogêneos invariantes, não constantes, têm grau no mínimo 2.

De fato, suponha que $p: \operatorname{Lie}(G) \cong \mathbb{R}^{n} \rightarrow \mathbb{R}$ é um polinômio linear homogêneo. Como $G$ é compacto, fazendo a média, podemos supor que a ação adjunta de $G$ em Lie $(G)$ age transformações ortogonais. Mas repare que o núcleo de p é um hiperplano G-invariante e, como a ação é ortogonal, o conjunto $V \subset \operatorname{Lie}(G)$ perpendicular ao núcleo de p é G-invariante. Ora, mas a ação de $G$ é ortogonal e, portanto, a ação de a $\in G$ em $V$ é por Id ou -Id. Porém, se fosse -Id não seria G-invariante. Logo $G$ age em $V$ como a identidade e todo $v \in V$ comuta com os elementos de $G$. Assim, dado $u \in \operatorname{Lie}(G)$, $\exp (t u) v \exp (-t u)=v$. Logo, derivando em $t=0$ dos dois lados, temos que $[u, v]=0$ e, portanto, o núcleo de Lie $(G)$ é não trivial e $G$ não é semi-simples.

Ou seja, as classes características de $\kappa_{G}\left(P_{j}\right)$ tem grau maior ou igual a 4 . Assim, se a base $M / G$ do fibrado principal tem dimensão menor que 3, então

$$
H^{*}(M / T) \cong H^{*}(M / G)\left[x_{1}, \cdots, x_{r}\right] / J
$$

onde $J$ é o ideal gerado apenas pelos $p_{1}, \cdots, p_{n}$ sem envolver os coeficientes de $M / G$ já que a imagem de $P_{1}, \cdots, P_{n}$ pelo homomorfismo característico é a 
classe de zero. Portanto,

$$
\begin{gathered}
H^{*}(M / T) \cong H^{*}(M / G)\left[x_{1}, \cdots, x_{r}\right] / J \cong H^{*}(M / G) \otimes\left(\mathbb{C}\left[x_{1}, \cdots, x_{r}\right] / J\right) \\
\cong H^{*}(M / G) \otimes H(G / T)
\end{gathered}
$$

pelos exemplos anteriores. Assim, como $M / T=(M \otimes G / T) / G$, basicamente estamos provando uma versão da fórmula de Künneth.

\section{Exemplo 8.4.7 (Fibrado Flag Homogêneo para $S O(3)$ sobre $\mathbb{S}^{4}$ ) $O$}

objetivo deste exemplo é basicamente dar uma expressão mais exata para o nosso teorema principal no caso de $S O(3)$-fibrados principais sobre $\mathbb{S}^{4}$ os classificando por meio de informações cohomológicas.

Repare primeiro que podemos cobrir a esfera $\mathbb{S}^{n}$ com duas cartas onde a interseção é uma vizinhança tubular do equador $\mathbb{S}^{n-1}$ tão pequena quanto se queira. Desta forma, o fibrado será dado pelo cociclo associado a este recobrimento, isto é, um mapa da vizinhança tubular do equador $\mathbb{S}^{n-1}$ no grupo estrutural, na verdade, como estaremos interessados na classe de homotopia do mapa e a vizinhança tubular se contrai ao equador, podemos dispensar a vizinhança e trabalhar apenas com o mapa de $\mathbb{S}^{n-1}$ ao grupo estrutural.

Afirmamos assim que temos uma bijeção entre os fibrados principais e as classes de homotopia de mapas $\mathbb{S}^{n-1}$ em $G$, isto é, $\Pi_{n-1}(G)$ (caso $n=2$ recaímos no grupo fundamental). Um dos lados é razoavelmente simples de se observar.

Primeiro, como $G$ representa o grupo estrutural do fibrado, temos o mapa de $\mathbb{S}^{n-1} \rightarrow G$, onde $G$ está contido no grupo de difeomorfismo da fibra que, nesse caso, também é $G$. Assim, um mapa $\alpha: \mathbb{S}^{n-1} \rightarrow G$ induz um difeomorfismo $\phi$ na fibra $G$. Agora consideremos $\mathbb{S}^{+}$o hemisfério superior da esfera $\mathbb{S}^{n}$ (incluindo o equador) e $\mathbb{S}^{-}$o hemisfério inferior (incluindo o equador) da esfera $\mathbb{S}^{n}$. Temos que $\mathbb{S}^{+} \times G$ e $\mathbb{S}^{-} \times G$ tem fronteira $\mathbb{S}^{n-1} \times G$, portanto fronteira compacta. Logo, existem vizinhanças tubulares das fronteiras difeomorfas a $[0,1) \times \mathbb{S}^{n-1} \times G$ e mergulhos/prolongamentos de $\mathbb{S}^{+} \times G$ em $(-1,1) \times \mathbb{S}^{+} \times G$ e de $\mathbb{S}^{-} \times G$ em $(-1,1) \times \mathbb{S}^{-} \times G$.

Mas repare que nossos fibrados principais são as colagens de $\mathbb{S}^{+} \times G e$ $\mathbb{S}^{-} \times G$ ao longo da fronteira $\mathbb{S}^{n-1} \times G$, portanto, o mapa $\phi$ induz o fibrado

$$
\mathbb{S}^{+} \times G \coprod \mathbb{S}^{-} \times G /[(t,(x, y)) \sim(t,(x, \phi(y))], \quad t \in(-1,1) .
$$


Logo, se $\alpha, \beta: \mathbb{S}^{n-1} \rightarrow G$ são homotópicos, temos um caminho $\gamma_{s}$ contínuo, onde $\gamma_{0}=\alpha$ e $\gamma_{1}=\beta$. Para cada s, temos induzido um difeomorfismo $\phi_{s}$ da fibra $G$ e, portanto, os mapas $\phi_{0} e \phi_{1}$, induzidos por $\alpha e$ $\beta$ respectivamente, são difeomorfismos isotrópicos. Logo, por um resultado geral de topologia diferencial, $\left(\mathbb{S}^{+} \times G \amalg \mathbb{S}^{-} \times G /\left[(t,(x, y)) \sim\left(t,\left(x, \phi_{0}(y)\right)\right]\right)\right.$ $e\left(\mathbb{S}^{+} \times G \amalg \mathbb{S}^{-} \times G /\left[(t,(x, y)) \sim\left(t,\left(x, \phi_{1}(y)\right)\right]\right)\right.$ são difeomorfos. Assim, os fibrados principais são isomorfos. Dessa forma, mapas homotópicos induzem fibrados principais isomorfos. Não iremos provar, mas a volta do resultado também é válido no nosso caso e, portanto, a bijeção é válida.

Então, quando $G=S O(3)$ e $\mathbb{S}^{n}=\mathbb{S}^{4}$, pelo comentado anteriormente, os fibrados principais correspondentes estão classificados pelas classes de homotopia de mapas

$$
\mathbb{S}^{3} \rightarrow S O(3)
$$

Por um resultado de homotopia estas classes estão em bijeção com os inteiros, na verdade, se temos um fibrado $M \rightarrow \mathbb{S}^{4}$ o polinômio $\mathcal{W}$ invariante $x^{2}$ (em SO(3) pelo feito nos exemplos anteriores) define, através do isomorfismo do teorema de Chevalley, a classe característica de $\kappa_{S O(3)}\left(x^{2}\right)$ em $H^{4}\left(\mathbb{S}^{4}, \mathbb{Z}\right)=$ $\mathbb{Z}$. Na verdade, aqui estamos afirmando que $0 \neq \kappa_{S O(3)}\left(x^{2}\right) \in \mathbb{Z}$, isto é, $x^{2}$, por meio do homomorfismo característico, gera $H^{4}\left(\mathbb{S}^{4}, \mathbb{Z}\right)$ (não iremos provar, mas uma maneira de verificar isso é primeiro usar que há uma bijeção entre $S O(3)$-fibrados principais sobre $\mathbb{S}^{4}$ e as classes de homotopias de mapas de $\mathbb{S}^{4}$ para o espaço de classificação $B(S O(3))$. Todavia, uma forma de definir as classes características é pelo pullback por um desses mapas de certas classes de homologia de $B(S O(3))$. Assim, definindo como c a classe característica, a idéia seria mostrar que o mapa classificante $f$ induz em cohomologia um mapa $f^{*}$ não nulo, isto é, $f^{*}\left(c\left(x^{2}\right)\right)$ é não nulo). Desta forma, os fibrados principais estão classificados pela classe característica em questão. Ou seja, consideremos

[clas. de isomor. de $S O(3)$-fibrados principais sobre $\left.\mathbb{S}^{4}\right]=P$,

temos o isomorfismo

$$
\begin{array}{lll}
P & \rightarrow & H^{4}\left(\mathbb{S}^{4}, \mathbb{Z}\right) \cong \mathbb{Z} \\
M & \mapsto & \kappa_{S O(3)}\left(x^{2}\right) .
\end{array}
$$

Assim, para qualquer fibrado $M$ como acima, temos que seu fibrado flag homogêneo $Y=M / T$ é

$$
H^{*}\left(\mathbb{S}^{4}\right)[x] /<x^{2}-\kappa_{S O(3)}\left(x^{2}\right)>
$$


Nomeando por $b$ o gerador $H^{4}\left(\mathbb{S}^{4}\right)$ (para uma orientação), temos $\kappa_{S O(3)}\left(x^{2}\right)=s b$ e, então, o anel $H^{*}(Y)$ vai ter geradores $b, x$, e o ideal do quociente é gerado por $b^{2}, s b-x^{2}$.

Após um longo trabalho para provar o teorema 3.1.3 e os exemplos de aplicação acima finalmente encerramos esta dissertação. 


\section{A}

\section{Prova do Teorema 2.2.9}

Como falamos no capítulo 2, a prova do teorema 2.2.9 se divide em duas partes, a primeira provar que $G / H$ tem base enumerável e é Hausdorff e a segunda mostrar que $G / H$ é localmente Euclidiano e que é possível cobrir $G / H$ por um sistema de coordenadas.

Para ver que $G / H$ tem base enumerável e é Hausdorff, temos que ver primeiro que o mapa $\pi$ é aberto, mas isso é consequência da topologia induzida por $G$. De fato, se $U \in G$ é aberto, temos que $a U=\{a b \in G \mid b \in U\}$ é aberto em G. Assim, como

$$
\pi^{-1}(\pi(U))=\cup_{a \in G} a U
$$

temos que $\pi(U)$ é aberto em $G / H$. Portanto, como $G$ tem base enumerável e $\pi$ é aberto, $G / H$ tem base enumerável. Além disso, novamente, como $\pi$ é aberto, mostrar que $G / H$ é Hausdorff se resume a mostrar que

$$
\Delta=\{(x, x) \in G / H \times G / H \mid x \in G / H\}
$$

é fechado em $G / H \times G / H$. Consideremos a projeção $t: G \times G \rightarrow G / H \times G / H$. Temos que $\Delta$ é fechado se $t^{-1}(\Delta)$ é fechado. Ora, mas $t(a, b) \in \Delta$ implica que $b^{-1} a \in H, \operatorname{logo} t^{-1}(\Delta)=\pi^{-1}(H)$. E como $H$ é fechado temos $\pi^{-1}(H)$ fechado.

Agora sim, o trabalho passa a ser provar que $G / H$ é localmente euclidiano e lhe dar um sistema de coordenadas. Mas fazer isso não é nada fácil, e para dar uma ideia da construção, faremos uso do teorema de Frobenius. Então façamos algumas recordações. Uma distribuição $D$ de dimensão $d$ em uma variedade $M$ de dimensão $m$ é uma escolha de um subespaço $D(p)$ de $T_{p} M$ com $p \in M$. Dizemos que $D$ é suave se para cada $p \in M$, existe vizinhança $U$ de $p$ tal que existem campos vetoriais de classe $C^{\infty}, \chi_{1}, \cdots, \chi_{d}$, em $U$ que geram (através de combinação linear) $D$ em cada ponto de $U$. Além disso, $D$ é dito involutiva se o colchete de Lie de $\chi$ e $\tilde{\chi}$ pertence a $D$ para todo $\chi, \tilde{\chi} \in D$. Repare que se $h$ é uma subálgebra de Lie de $G$, então o subconjunto

$$
D=\cup_{a \in G} D_{a} \subset T G
$$


onde

$$
D_{a}=\left\{\chi_{a} \mid \chi \in h\right\} \subset T_{a} G
$$

é uma distribuição involutiva em $G$, em particular, também é invariante a esquerda. A prova mais precisa deste pode ser vista em [Lee].

Além disso, uma subvariedade integral de $D$ é uma imersão $\phi: N \rightarrow M$ tal que para todo $c \in N$

$$
d \phi\left(T_{c} N\right)=D(\phi(c))
$$

Por [War], se $D$ é uma distribuição suave em $M$ tal que em cada ponto de $M$ passa uma variedade integral de $M$, então $D$ é involutiva.

Fica claro pelas observações anteriores que iremos aplicar isto diretamente no subgrupo de Lie $H$ (e, portanto, sua álgebra de Lie $h$ ) de $G$. Esta aplicação virá como resultado do teorema de Frobenius, que diz que dados $D$ uma distribuição $C^{\infty}$ de dimensão $d$ de $M$ com dimensão $m$ e $p \in M$, então existe uma variedade integral de $D$ passando por $p$. Ou ainda mais, existe um sistema de coordenadas $(U, \phi)$ que é centrado em $p$, com funções coordenadas $x_{1}, \cdots, x_{m}$ tal que as fatias

$$
x_{i}=\text { constante, para cada } i \in\{d+1, \cdots, m\}
$$

são variedades integráveis de $D$. E ainda, se $(N, \phi)$ é uma variedade integral conexa de $D$ tal que $\phi(n) \subset U$, então $\phi(N)$ está em uma destas fatias. Seguiremos então o livro [War] para provar o resultado.

Assim, considerando $D$ a distribuição de $G$ determinada pela álgebra de Lie sobre $H$. Temos então, pelo teorema anterior, um sistema de coordenadas cúbicas $(V, \phi)$ centrada em $e \in G$, com funções coordenadas $x_{1}, \cdots, x_{n}$ como acima. Podemos escolher $V$ pequeno o suficiente para que $V \cap H=S_{0}$ onde $S_{0}$ é a fatia sobre $e$, ou seja, onde está a componente conexa de $e$ em $G$. Apesar $(V, \phi)$ ser um sistema de coordenadas interessante, não é ele que estamos buscando, mas partimos dele. Queremos encontrar uma vizinhança que seja invariante em relação as classes de $G / H$. Consdere $W$ e $U$ vizinhanças de $e$, tal que $W W \subset V$ e $U^{-1} U \subset W$, onde $U^{-1} U=\left\{a^{-1} b \mid a, b \in U\right\}$.

Assim, se $\sigma \in \tau H$ e $\sigma, \tau \in U$, temos que

$$
\tau^{-1} \sigma \in W \cap H=W \cap S_{0}
$$

Logo, como $\tau\left(W \cap S_{0}\right)$ é conexo e $\tau\left(W \cap S_{0}\right)$ é uma variedade integral de $D$, 
temos que $\tau\left(W \cap S_{0}\right)$ está contido em uma única fatia de $V$. Como, pelo feito acima, $\sigma \in \tau\left(W \cap S_{0}\right)$, temos que $\sigma$ e $\tau$ estão na mesma fatia. Ou seja, o sistema de coordenadas que buscamos é $(U, \phi)$.

Precisamos agora ligar diretamente a construção que fizemos com a imagem do mapa $\pi$, para isso, considere $S \subset \mathbb{R}^{d}$ a fatia de $\phi(U)$ no qual as coordenadas $x_{d+1}, \cdots, x_{m}$ desaparecem. Pela própria escolha de $(U, \phi)$, temos que

$$
\bar{\phi}^{-1}=\left.\pi \circ \phi^{-1}\right|_{S}: S \rightarrow \pi(U)
$$

é bijetivo, além de ser contínuo e aberto, logo um homeomorfismo. Assim temos um novo mapa

$$
\bar{\phi}: \pi(U) \rightarrow S
$$

que iremos trabalhar. Na verdade, o que simplesmente fizemos foi pegar $(U, \phi)$ e levar em $(\phi(U), \bar{\phi})$ que é mais adequado para a situação, um sistema de coordenadas sobre a identidade de $G / H$. É claro que precisamos estender para os outros pontos de $G / H$ e faremos isso através da translação a esquerda em $G$, ou mais precisamente, o homeomorfismo de $G / H$ induzido pela translação a esquerda de $G$. Seja $a \in G$, definimos:

$$
\bar{L}_{a}: G / H \rightarrow G / H
$$

tal que $\bar{L}_{a}(b H)=a b H$.

Agora estamos preparados para construir um sistema de coordenadas sobre qualquer ponto $a H \in G / H$, basta considerar o mapa

$$
\bar{\phi}_{a H}=\left.\bar{\phi} \circ \bar{L}_{a^{-1}}\right|_{\bar{L}_{a}}(\pi(U))
$$

e assim temos que $\left(\bar{L}_{a}(\pi(U)), \bar{\phi}_{a H}\right)$ é um sistema de coodenadas sobre $a H$.

Repare que até aqui não trabalhamos com estruturas diferenciáveis, o que iremos fazer agora. Consideremos uma maximilização da coleção

$$
\left\{\bar{L}_{a}(\pi(U)), \bar{\phi}_{a H} \mid a \in G\right\}
$$

de sistemas de coordenadas. Verifiquemos a diferenciabilidade das interseções e assim teremos construído uma estrutura diferenciável em $G / H$. Sejam $\left(\bar{L}_{a}(\pi(U)), \bar{\phi}_{a H}\right)$ e $\left(\bar{L}_{b}(\pi(U)), \bar{\phi}_{b H}\right)$ dois desses sistemas de coordenadas e $Z=\bar{\phi}_{a H}\left(\bar{L}_{a}(\pi(U)) \cap \bar{L}_{b}(\pi(U))\right)$. Provemos que $\left.\bar{\phi}_{b H} \circ \bar{\phi}_{a H}^{-1}\right|_{Z}$ é $C^{\infty}$. 
Fixe $z \in Z$. Temos que $\bar{L}_{b^{-1} H} \circ \bar{L}_{a H} \circ \bar{\phi}^{-1}(z) \in \pi(U)$. Assim, por definição dos mapas, existe $k \in H$ tal que $b^{-1} a \phi^{-1}(z) k \in U$. E como $U$ é aberto, existe vizinhança $W$ de $z$ em $Z$ tal que $b^{-1} a \phi^{-1}(W) k \subset U$. Como a diferenciabilidade é local, em vez de $Z$, mostremos que o mapa $\bar{\phi}_{b H} \circ \bar{\phi}_{a H}^{-1}$ é $C^{\infty}$ quando restrito a $W$.

Ora, mas por contrução, restrito a $W$ temos a igualdade

$$
\bar{\phi}_{b H} \circ \bar{\phi}_{a H}^{-1}=q_{0} \circ \phi \circ R_{k} \circ L_{b^{-1} a} \circ \phi^{-1}
$$

onde $q_{0}$ é a projeção canônica de $\phi(U)$ em $S$ (uma projeção do tipo ortogonal). De fato, como estamos nos restringindo a $W$ temos

$$
\begin{gathered}
\bar{\phi}_{b H} \circ \bar{\phi}_{a H}^{-1}=\bar{\phi} \circ \bar{L}_{b^{-1}} \circ\left(\bar{\phi} \circ \bar{L}_{a^{-1}}\right)^{-1}=\bar{\phi} \circ \bar{L}_{b^{-1}} \circ \bar{L}_{a} \circ \bar{\phi}^{-1}= \\
=\bar{\phi} \circ \pi \circ L_{b^{-1} a} \circ R_{k} \circ \phi^{-1}=q_{0} \circ \phi \circ R_{k} \circ L_{b^{-1} a} \circ \phi^{-1} .
\end{gathered}
$$

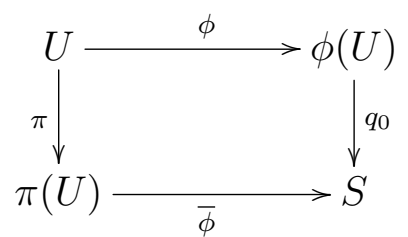

Assim, como todos os mapas do lado direito são $C^{\infty}$, temos que $\bar{\phi}_{b H} \circ \bar{\phi}_{a H}^{-1}$ restrito a $W$ é $C^{\infty}$ e, portanto, temos uma estrutura diferenciável para $G / H$.

E ainda, com esta estrutura, $\pi: G \rightarrow G / H$ é $C^{\infty}$, pois restrito a um aberto da forma $L_{a}(U)$ é igual a composição $\left.\bar{\phi}_{a H}^{-1} \circ q_{0} \circ \phi \circ L_{a^{-1}}\right|_{L_{a}(U)}$, como podemos ver no diagrama acima, que são mapas $C^{\infty}$. Então, com a estrutura que criamos, $\pi: G \rightarrow G / H$ é uma submersão.

E assim, provamos o seguinte:

Se $G$ é um grupo de Lie e $H<G$ é um subgrupo fechado, então existe uma estrutura de variedade em $G / H$ compatível com a topologia quociente na qual a projeção canônica $\pi: G \rightarrow G / H$ é uma submersão. 
B

\section{Teorema Equivariante de de Rham}

O teorema diz o seguinte:

Teorema B.0.1 Seja G um grupo de Lie compacto agindo sobre uma variedade compacta suave M. Então

$$
H_{G}^{*}(M)=H_{G}(\Omega(M)) .
$$

Qualquer grupo de Lie compacto pode ser visto como um subgrupo fechado de $U(n)$. Desta forma, é suficiente usar $U(n)$ para facilitar nos cálculos.

Defina como sendo $C^{\infty}$ o espaço de todas as sequências $\left(z_{1}, \cdots, z_{n}, \cdots\right)$ com $z_{i}=0$ para $i$ suficientemente grande. Portanto $C^{\infty}=\cup C^{k}$ onde $C^{k}$ consiste de todas as sequências com $z_{i}=0$ se $i>k$.

Agora, considere $\mathcal{E}=\mathcal{E}^{n}$ o conjunto de todas as $n$-tuplas $\nu=$ $\left(v_{1}, \cdots, v_{n}\right)$ com $v_{i} \in C^{\infty}$. Agora, para cada $k>n$ seja $\mathcal{E}_{k}$ o conjunto de todas as $n$-tuplas ortornormais (pelo produto Hermitiano clássico) com $v_{i} \in C^{k}$. $\mathrm{E}$, é claro, como temos a inclusão natural de $C^{k}$ em $C^{k+1}$ dado pelo mapa $\left(z_{1}, \cdots z_{k}\right) \mapsto\left(z_{1}, \cdots, z_{k}, 0\right)$, temos a inclusão

$$
i_{k}: \mathcal{E}_{k} \rightarrow \mathcal{E}_{k+1}
$$

Repare ainda que podemos compô-las para gerar os mapas

$$
j_{k, m}: \mathcal{E}_{k} \rightarrow \mathcal{E}_{m}
$$

onde $m>k$. Desta forma, também temos a inclusão

$$
j_{k}: \mathcal{E}_{k} \rightarrow \mathcal{E}
$$

Façamos agora algumas considerações a respeito do espaço construído. As inclusões induzem uma topologia em $\mathcal{E}$, a topologia final, neste sentido dizemos que $U \in \mathcal{E}$ é aberto se, e somente se, cada uma dos subconjuntos $j_{k}^{-1}(U)$ é aberto. Desta forma, uma série de pontos converge se, e somente se, todos os pontos estejam em algum $\mathcal{E}_{k}$. Com isto, se $f$ é um mapa contínuo 
de um espaço topológico compacto $X$ em $\mathcal{E}$, então $f(X) \subset \mathcal{E}_{k}$ para algum $k$ já que $f(X)$ é compacto e, portanto, toda sequência admite subsequência convergente, o que não seria possivel caso $f(X)$ não estivesse contido em nenhum $\mathcal{E}_{k}$ pela topologia final.

Outro ponto que temos que ressaltar é que, assim como fizemos e verificamos no capítulo 3 , se $A=\left[a_{i j}\right] \in U(n)$ e $\nu=\left(v_{1}, \cdots, v_{n}\right) \in \mathcal{E}$, a ação de $U(n)$ em $\mathcal{E}$ dada por

$$
A \nu=\eta=\left(w_{1}, \cdots, w_{n}\right)
$$

onde $w_{i}=\sum_{j} a_{i j} v_{j}$, é uma ação livre.

Além disso, um ponto interessante é que, apesar de $\mathcal{E}$ não ser uma variedade diferenciável, $\mathcal{E}$ é limite de uma sequência de variedades diferenciáveis, isto é, $\mathcal{E}_{k}$, para todo $k>n$, é uma variedade diferenciável. De fato, $\mathcal{E}_{K}$ tem uma estrutura Grassmanniana em que podemos ver $\mathcal{E}$ mergulhado em $\operatorname{Gr}(n, K)$, isto é,

$$
\mathcal{E}_{k}=(U(K) / \sim) \subset G r(n, k)
$$

onde $\sim=(I d \times U(k-n))$. Assim, como uma Grassmanniana é uma variedade compacta, $\mathcal{E}_{k}$ é também uma variedade compacta.

Esta estrutura também tem uma propridade natural, a fibração dada por

$$
\gamma: \mathcal{E}_{k}^{(n)} \rightarrow \mathcal{E}_{k}^{(n+1)},\left(v_{1}, \cdots, v_{n}\right) \mapsto\left(v_{1}, \cdots, v_{n-1}\right)
$$

gera um fibrado esférico, isto é, cada fibra é uma esfera, sendo mais preciso a esfera $\mathbb{S}^{2(k-(n-1))-1}$. O fato de termos a multiplicação por 2 no índice da esfera se deve a estarmos trabalhando com entradas em $\mathbb{C}$, não em $\mathbb{R}$. Desta forma, usando a representação canônica de $\mathbb{C}$ em $\mathbb{R}^{2}$ temos a passagem. Além disso, o fato de ser uma esfera é natural já que as tuplas são ortornomais.

Teorema B.0.2 Seja X uma variedade compacta de dimensção m. Todo mapa contínuo $f: X \rightarrow \mathcal{E}$ é contrátil a um ponto.

Prova: Repare primeiro que como $f(X) \subset \mathcal{E}_{k}$ para algum $k$, basta provar então que o mapa $f: X \rightarrow \mathcal{E}_{k}=\mathcal{E}_{k}^{(n)}$ é contrátil a um ponto se $k \geq m+n$. Considere então a fibração

$$
\gamma: \mathcal{E}_{k}^{(n)} \rightarrow \mathcal{E}_{k}^{n-1}
$$


como já vimos anteriormente.

Note que $\gamma \circ f$ é homotópico a $\gamma \circ \gamma \circ f$. Assim, indutivamente, $\gamma \circ f$ é homotópico ao mapa $\alpha: X \rightarrow \mathcal{E}_{k}^{(1)}$, tal que $\alpha\left(v_{1}, \cdots, v_{n}\right)=\left(v_{1}\right)$, que é homotópico ao mapa constante pelo espaço ser contrátil. Logo $\gamma \circ f$ é contrátil.

Assim, pelo levantamento como na propriedade de homotopia de cobertura de $[\mathrm{BT}]$,
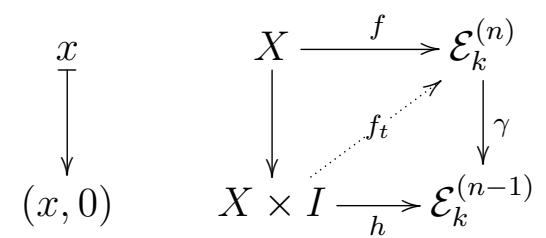

temos que $f$ é homotópico a um mapa $h: X \rightarrow \mathcal{E}_{k}^{(n)}$ que tem imagem na fibra sobre um ponto, fibra esta, dada por $\gamma$. Como já vimos, a fibra é a esfera $\mathbb{S}^{2(k-(n-1))-1}$, logo a imagem por $h$ está em $\mathbb{S}^{2(k-(n-1))-1}$. Ora, mas $k \geq n+m$ por hipótese, portanto

$k-(n-1)>m \geq 0 \Longrightarrow 2(k-(n-1))>m+1 \Longrightarrow 2(k-(n-1))-1>m$.

Por hipótese $X$ é compacto e, além disso, $\mathbb{S}^{2(k-(n-1))-1}$ também é compacto, assim como podemos ver em [Hatc], $X$ e $\mathbb{S}^{2(k-(n-1))-1}$ tem estrutura de $C W$ complexo. Segue pelo teorema de aproximação celular, também em [Hatc], que $h$ é homotópico a um mapa celular $\lambda$. Ou seja, o esqueleto de $X$ está com a imagem, por $\lambda$, contida no esqueleto da mesma dimensão em $\mathbb{S}^{2(k-(n-1))-1}$ pois, como vimos, $\mathbb{S}^{2(k-(n-1))-1}$ tem dimensão superior a dimensão de $X$. Portanto, existe pelo menos um ponto em $\mathbb{S}^{2(k-(n-1))-1}$ que não está na imagem de $\lambda$. Logo a imagem de $\lambda$ é contrátil e como consequência, $\lambda$ e, portanto, $f$ é contrátil a um ponto.

Como já vimos, cada $\mathcal{E}_{k}$ admite uma estrutura de variedade, desta forma iremos definir o conjunto $\Omega(\mathcal{E})$ como sendo o limite inverso da sequência de projeções abaixo:

$$
\cdots \longleftarrow \Omega\left(\mathcal{E}_{k}\right) \longleftarrow \Omega\left(\mathcal{E}_{k+1}\right) \longleftarrow \Omega\left(\mathcal{E}_{k+2}\right) \longleftarrow \cdots
$$

Agora, para o que queremos sobre $\Omega(\mathcal{E})$, temos que mostrar que $\Omega(\mathcal{E})$ satisfaz a condição $(\mathbf{C})$ e que é acíclico. 
Teorema B.0.3 $\Omega(\mathcal{E})$ é acíclico.

Antes de atacar diretamente o problema, lembremos o teorema de de Rham:

Teorema B.0.4 (Teorema de de Rham) Se $M$ é uma variedade, então a cohomologia de de Rham de $M$ é isomorfa a cohomologia singular de $M$ calculada sobre $\mathbb{R}$, isto é,

$$
H_{d r}^{n}(M)=H_{\text {sing }}^{n}(M, \mathbb{R}),
$$

onde $H_{\text {sing }}^{*}$ representa a cohomologia singular.

Este resultado é interessante no nosso caso pois, como vimos, $\mathcal{E}_{k}$ para cada $k$ é uma variedade. Vale ainda ressaltar que, como $\mathcal{E}_{k}$ é compacto, e, portanto, admite estrutura de $C W$ complexo, podemos ver a cohomologia singular como sendo simplesmente a cohomologia simplicial.

Vamos então a prova do Teorema B.0.3.

Prova: Lembremos primeiro, como podemos ver em [Hatc], que para construir o esqueleto de $\mathcal{E}$, consideramos mapas continuos $f: \triangle^{m} \rightarrow \mathcal{E}_{k}^{n}$. Mas repare que $\triangle^{m}$ nada mais é que uma variedade compacta de dimensão $m$. Desta forma, pelo teorema B.0.2, o mapa $f$ é contrátil a um ponto para $k>m$ suficientemente grande, ou seja,

$$
H_{\text {sing }}^{m}\left(\mathcal{E}_{k}\right)=0
$$

Agora, pelo teorema B.0.4, o resultado também vale para a cohomologia de de Rham, $H^{m}\left(\mathcal{E}_{k}\right)=H_{d R}^{m}\left(\mathcal{E}_{k}\right)=0$ para $k>m$ suficientemente grande. Neste caso, considere $\mu \in \Omega^{m}(\mathcal{E})$ uma forma fechada. Portanto, para $k$ suficientemente grande,

$$
\mu_{k}:=j_{k}^{*} \mu=d \nu_{k}
$$

Afirmamos, agora, que é possível escolher $\nu_{k}$ consistente na medida que $\nu_{k}=i_{k}^{*} \nu_{k+1}$, onde $\nu_{k+1}$ é tal que $\mu_{k+1}=j_{k+1}^{*} \mu=d \nu_{k+1}$.

De fato, pois para qualquer escolha de $\nu_{k+1}, i_{k}^{*} \nu_{k+1}$ e $\nu_{k}$ diferem por uma forma exata, digamos $d \vartheta$. Assim, podemos escolher uma $(m-2)$-forma em $\mathcal{E}_{k+1}$, $\beta$, tal que $i_{k}^{*} \beta=\vartheta$. Ou seja, trocando nosso $\nu_{k+1}$ inicial por $\nu_{k+1}+d \beta$ nos dá a escolha consistente que queríamos, já que, $i_{k}^{*}\left(\nu_{k+1}-d \beta\right)=\nu_{k}+d \lambda-d i_{k}^{*} \beta=\nu_{k}$. Assim, indutivamente podemos considerar uma escolha consistente para $k$ 
suficientemente grande.

Desta forma, podemos sempre considerar um $\nu \in \Omega(\mathcal{E})$ tal que $j_{k}^{*} \nu=\nu_{k}$ e $d \nu=\mu$.

Logo, como para todo $m>0$ considerando um $k$ suficientemente grande $H^{m}\left(\mathcal{E}_{k}\right)=0, \Omega(\mathcal{E})$ sendo o limite inferior nos permite garantir que toda forma fechada é exata. Portanto $\Omega(\mathcal{E})$ é acíclico.

Assim, como $\mathcal{E}$ é acíclico e a ação de $U(n)$ em $\mathcal{E}$ é livre, temos na cohomologia equivariante topológica que:

$$
H_{G}^{*}(M)=H^{*}((M \times \mathcal{E}) / G)
$$

Queremos agora mostrar que $\Omega(\mathcal{E})$ satisfaz a condição $(\mathbf{C})$ para definir a cohomologia equivariante de $G^{*}$-módulo.

Teorema B.0.5 $\Omega(\mathcal{E})$ satisfaz a propriedade $(\boldsymbol{C})$.

Prova: Sejam $z_{i j}$ as funções definidas em $\mathcal{E}$, estabelecendo $z_{i j}(\nu)$ como a i-ésima coordenada do vetor $\nu=\left(v_{1}, \cdots, v_{n}\right)$ e $Z$ a matriz com entradas $z_{i j}$. Então, $Z$ tem um número finito de entradas diferentes de zero quando avaliadas em cada $\mathcal{E}_{k}$. Isto é,

$$
\begin{gathered}
j_{k}: \mathcal{E}_{k} \rightarrow \mathcal{E} \\
z \mapsto z,
\end{gathered}
$$

considerando $Z_{k}$ uma avaliação de $Z$ em $\mathcal{E}_{k}$, temos que

$$
Z_{k}=\left(\begin{array}{ccc}
z_{11} & \cdots & z_{n 1} \\
\vdots & \ddots & \vdots \\
z_{1 k} & \cdots & z_{n k} \\
0 & \cdots & 0 \\
\vdots & \ddots & \vdots
\end{array}\right)
$$

Como apenas um número finito de linhas é diferente de 0 , a matriz

$$
\Theta_{k}:=Z^{t} d \bar{Z}=
$$




$$
\left(\begin{array}{ccccc}
z_{11} & \ldots & z_{1 k} & 0 & \ldots \\
\vdots & & \vdots & \ldots & \ddots \\
z_{n 1} & \ldots & z_{n k} & 0 & \ldots
\end{array}\right) \cdot\left(\begin{array}{ccc}
d \bar{z}_{11} & \ldots & d \bar{z}_{n 1} \\
\vdots & \ddots & \vdots \\
d \bar{z}_{1 k} & \ldots & d \bar{z}_{n k} \\
0 & \ldots & 0 \\
\vdots & \ddots & \vdots
\end{array}\right)
$$

está bem definida. Mas repare que $\left(Z_{k}^{t} d \bar{Z}_{k}\right)=i_{k}^{*}\left(Z_{k+1}^{t} d \bar{Z}_{k+1}\right)$. Desta forma, $\Theta_{k} \in M(n \times n)\left(\Omega^{1}(\mathbb{C})\right)$, a matriz

$$
\Theta=\lim _{k \rightarrow \infty} \Theta_{k}
$$

está bem definida e cada componente $\Theta_{i j}=\lim _{\longleftarrow}\left(\Theta_{k}\right)_{i j}$ é uma 1-forma em $\mathcal{E}$.

Agora, como estamos considerando, em hipótese, a ação de $U(n)$ em $\mathcal{E}$, temos que mostrar que $\Theta_{i j}$ representa a ação de $u(n)$, a álgebra de Lie de $U(n)$. Ora, mas $u(n)$ pode ser definida pelas matrizes que somadas com a suas transpostas conjugadas é igual a 0 . Ora, mas pela construção de $Z_{k}$ temos que

$$
Z_{k}^{t} \bar{Z}_{k}=I d
$$

portanto

$$
d Z_{k}^{t} \bar{Z}_{k}+Z_{k}^{t} d \bar{Z}_{k}=0
$$

e, então,

$$
\Theta_{i j}+\bar{\Theta}_{j i}=0
$$

Segue então que $\Theta_{i j}$ representa uma ação de $u(n)$. E, é claro, pela definição de $z_{i j}$ e, portanto $Z$, temos que

$$
\iota_{a b}\left(\Theta_{i j}\right)=\Theta_{i j}\left(\xi_{a b}\right)=\delta_{a b}^{i j}
$$

Logo, $\Theta_{i j}$ são os elementos que fazem $\Omega(\mathcal{E})$ satisfazer a condição $(\mathbf{C})$ e, portanto, $\Omega(\mathcal{E})$ é acíclico.

Provemos agora o seguinte:

\section{Teorema B.0.6}

$$
H^{*}((M \times \mathcal{E}) / G)=H_{\text {bas }}(\Omega(M \times \mathcal{E})) .
$$

Prova: 
Ora, mas repare que, como $\mathcal{E}_{k}$ é uma variedade, então, pelo desenvolvimento de formas básicas em variedades na seção 5.4, temos que

$$
H^{i}\left(\left(M \times \mathcal{E}_{k}\right) / G\right)=H^{i}\left(\Omega\left(M \times \mathcal{E}_{k}\right)_{b a s}\right) .
$$

Logo, como as sequências

$$
\cdots \longleftarrow H^{i}\left(\left(M \times \mathcal{E}_{k}\right) / G\right) \longleftarrow H^{i}\left(\left(M \times \mathcal{E}_{k+1}\right) / G\right) \longleftarrow \cdots
$$

e

$$
\cdots \longleftarrow H^{i}\left(( \Omega ( M \times \mathcal { E } _ { k } ) _ { b a s } ) \longleftarrow H ^ { i } \left(\left(\Omega\left(M \times \mathcal{E}_{k+1}\right)_{b a s}\right) \longleftarrow \cdots\right.\right.
$$

são iguais termo a termo, temos o isomorfismo no limite inferior.

Apesar do teorema anterior ser interessante, ele nos dá a igualdade entre $H^{*}((M \times \mathcal{E}) / G)$ e $H_{\text {bas }}(\Omega(M \times \mathcal{E}))$ e não a de $H^{*}((M \times \mathcal{E}) / G)$ e $H_{\text {bas }}((\Omega(M) \times \Omega(\mathcal{E})))$ que precisamos para provar a igualdade entre as cohomologias equivariantes. Então provemos o seguinte:

Teorema B.0.7 A inclusão

$$
\Omega(M) \otimes \Omega(\mathcal{E}) \rightarrow \Omega(M \times \mathcal{E})
$$

induz um isomorfismo na cohomologia:

$$
H\left((\Omega(M) \otimes \Omega(\mathcal{E}))_{\text {bas }}\right) \rightarrow H\left(\left(\Omega(M \times \mathcal{E})_{\text {bas }}\right) .\right.
$$

Prova: Por um argumento de sequência espectral, como podemos ver no capítulo 8, é suficiente mostrar o resultado para a cohomologia usual, isto é, basta mostrar que a inclusão induz o isomorfismo

$$
H(\Omega(M) \otimes \Omega(\mathcal{E})) \rightarrow H(\Omega(M \times \mathcal{E})) .
$$

Ora, mas a cohomologia de $\Omega(\mathcal{E})$ é trivial pela acíclicidade e pela contratibilidade de $\mathcal{E}$, a cohomologia de $\Omega(M \times \mathcal{E})$ é a cohomologia de $\Omega(M)$, logo a igualdade é imediata.

E assim, por fim temos a igualdade:

$$
H_{G}(\Omega(M))=H_{G}^{*}(M) .
$$




\section{C}

\section{Fórmulas do Capítulo 7}

Provemos agora as equações:

$$
\begin{gathered}
a d \gamma\left(\iota_{b} \otimes 1\right)=-1 \otimes \iota_{b} \\
a d \gamma\left(\nu \otimes \iota_{b}\right)=0 \forall \nu \in A \\
(a d \gamma)^{2}\left(\iota_{b} \otimes 1\right)=0 \\
a d \gamma(d)=-d \theta^{a} \otimes \iota_{a}+\theta^{a} \otimes \mathcal{L}_{a} \\
(a d \gamma)^{2}(d)=-c_{a b}^{k} \theta^{a} \theta^{b} \\
(a d \gamma)^{3} d=0
\end{gathered}
$$

referentes ao capítulo 7 .

Lembremos que pela fórmula de produto, em um produto tensorial temos que

$$
\left(x_{1} \otimes y_{1}\right)\left(x_{2} \otimes y_{2}\right)=(-1)^{p q} x_{1} x_{2} \otimes y_{1} y_{2},
$$

onde $\operatorname{deg} y_{1}=p$ e $\operatorname{deg} x_{2}=q$. Assim, como $\gamma=\theta^{a} \otimes \iota_{a}, \operatorname{deg} \theta^{a}=1$ e $\operatorname{deg} \iota_{a}=-1$, provemos as equações.

Prova: [7.1.4]

$$
\gamma\left(\iota_{b} \otimes 1\right)=\left(\theta^{a} \otimes \iota_{a}\right)\left(\iota_{b} \otimes 1\right)=(-1) \theta^{a} \iota_{b} \otimes \iota_{a}
$$

e

$$
\left(\iota_{b} \otimes 1\right)\left(\theta^{a} \otimes \iota_{a}\right)=(-1)^{0} \iota_{b} \theta^{a} \otimes \iota_{a} .
$$

Logo,

$$
a d \gamma\left(\iota_{b} \otimes 1\right)=\left[\gamma, \iota_{b} \otimes 1\right]=-\left[\theta^{a}, \iota_{b}\right] \otimes \iota_{a}=-\delta_{b}^{a} \otimes \iota_{a}=-1 \otimes \iota_{b} .
$$


Prova: [7.1.5]

Suponha que $\nu \in A_{m}$, então

$$
\left(\theta^{a} \otimes \iota_{a}\right)\left(\nu \otimes \iota_{b}\right)=(-1)^{m} \theta^{a} \nu \otimes \iota_{a} \iota_{b}
$$

$\mathrm{e}$

$$
\left(\nu \otimes \iota_{b}\right)\left(\theta^{a} \otimes \iota_{a}\right)=(-1) \nu \theta^{a} \otimes \iota_{b} \iota_{a} .
$$

Ora, mas

$$
\left[\nu, \theta^{a}\right]=\nu \theta^{a}-(-1)^{m} \theta^{a} \nu
$$

e como, em particular, $A$ como um $W^{*}$-módulo é uma super álgebra comutativa, $\left[\nu, \theta^{a}\right]=0$, e, portanto,

$$
\nu \theta^{a}=(-1)^{m} \theta^{a} \nu
$$

Assim,

$$
-\nu \theta^{a} \otimes \iota_{b} \iota_{a}=(-1)^{m+1} \theta^{a} \nu \times \iota_{b} \iota_{a} .
$$

Logo,

$$
a d \gamma\left(\nu \times \iota_{b}\right)=\left[\gamma, \nu \times \iota_{b}\right]=(-1)^{m} \theta^{a} \nu \times\left[\iota_{a}, \iota_{b}\right]=0
$$

e assim provamos a equação 7.1.5.

Prova: [7.1.6]

$$
(a d \gamma)^{2}\left(\iota_{b} \otimes 1\right)=\left[\gamma,\left[\gamma, \iota_{b} \otimes 1\right]\right]=\left[\gamma,-1 \otimes \iota_{b}\right]
$$

por 7.1.4. Assim, por 7.1.5, temos que

$$
(a d \gamma)^{2}\left(\iota_{b} \otimes 1\right)=0
$$

provando assim a equação 7.1.6.

Prova: [7.1.7]

$$
\begin{gathered}
a d \gamma(d)=a d \gamma\left(d_{A} \otimes 1+1 \otimes d_{b}\right)=\left[\theta^{a} \otimes \iota_{a}, d_{A} \otimes 1+1 \otimes d_{b}\right]= \\
=\left[\theta^{a} \otimes \iota_{a}, d_{A} \otimes 1\right]+\left[\theta^{a} \otimes \iota_{a}, 1 \otimes d_{b}\right]=-(1)^{-1 \cdot 1}\left[\theta^{a}, d\right] \otimes \iota_{a}+(-1)^{0} \theta^{a} \otimes\left[\iota_{a}, d\right]= \\
=-d \theta^{a} \otimes \iota_{a}+\theta^{a} \otimes \mathcal{L}_{a} .
\end{gathered}
$$


Logo, a equação 7.1 .7 é verdadeira.

Prova: [7.1.8]

Por 7.1.7,

$$
(a d \gamma)^{2}(d)=a d \gamma\left(-d \theta^{a} \otimes \iota_{a}+\theta^{a} \otimes \mathcal{L}_{a}\right) .
$$

Assim,

$$
(a d \gamma)^{2}(d)=a d \gamma\left(-d \theta^{a} \otimes \iota_{a}\right)+a d \gamma\left(\theta^{a} \otimes \mathcal{L}_{a}\right)
$$

e, por 7.1.5,

$$
a d \gamma\left(d \theta^{a} \otimes \iota_{a}\right)=0 .
$$

Logo,

$$
\begin{gathered}
(a d \gamma)^{2}(d)=\left[\theta^{b} \otimes \iota_{b}, \theta^{a} \otimes \mathcal{L}_{a}\right]=(-1)^{1} \theta^{b} \theta^{a} \times \iota_{b} \mathcal{L}_{a}-(-1)^{0} \theta^{a} \theta^{b} \otimes \mathcal{L}_{a} \iota_{b}= \\
=-\theta^{a} \theta^{b} \otimes\left[\mathcal{L}_{a}, \iota_{b}\right]=-c_{a b}^{k} \theta^{a} \theta^{b} \otimes \iota_{k} .
\end{gathered}
$$

Assim, concluímos a equação 7.1.8.

Prova: [7.1.9]

Por 7.1.8,

$$
(a d \gamma)^{3}(d)=a d \gamma\left(-c_{a b}^{k} \theta^{a} \theta^{b} \otimes \iota_{k}\right)
$$

e por 7.1.5, temos que

$$
\operatorname{ad\gamma }\left(\theta^{a} \theta^{b} \otimes \iota_{k}\right)=0
$$

Logo,

$$
(a d \gamma)^{3}(d)=0
$$

e a equação 7.1 .9 é satisfeita.

Com isto concluímos a prova das equações 7.1.4, 7.1.5, 7.1.6, 7.1.7, 7.1.8 e 7.1.9. 
D

\section{Sequência Espectral de Complexo Duplo}

No capítulo 7 falamos um pouco sobre filtração, iremos rever o uso desse conceito logo à frente. Neste momento, chamaremos a atenção para o fato de que a graduação dada pela filtração também pode agir de forma funtorial. Isto é, consideremos $A$ e $A^{\prime}$ álgebras e $\mathcal{F}=\left\{F_{n}\right\}_{n \in \mathbb{Z}} \mathcal{F}^{\prime}=\left\{F_{n}^{\prime}\right\}_{n \in \mathbb{Z}}$ suas filtrações respectivamente, então, se $f$ é uma função que preserva a filtração, segue que o diagrama

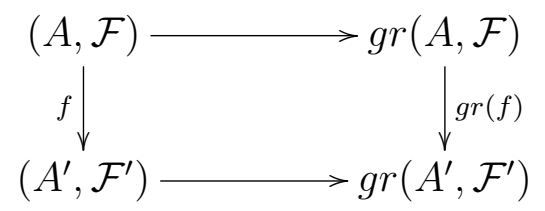

é comutativo. Na segunda seção deste apêndice, veremos o funtor graduação na perspectiva de sequências espectrais.

\section{D.1}

\section{Construção da Sequência Espectral de Complexos Duplos}

Antes de começar a falar propriamente sobre filtração em complexo duplos precisamos definir o que seria complexo duplo. Um complexo duplo é um espaço vetorial bigraduado

$$
C=\underset{p, q \in \mathbb{Z}}{\bigoplus} C^{p, q}
$$

com operadores fronteira

$$
d: C^{p, q} \longrightarrow C^{p, q+1}, \quad \delta: C^{p, q} \longrightarrow C^{p+1, q}
$$

que satisfazem:

$-d^{2}=0$

$-\delta^{2}=0$

$-d \delta+\delta d=0$.

Pelas duas primeiras propriedades, ao fixar $p$ ou $q, C^{p, q}$ se torna um complexo de cadeia em relação a $d$ ou a $\delta$ respectivamente. Além disso, graças às três 
propriedades, temos que $(d+\delta)^{2}=0$, isto nos permite estudar o complexo total associado definido por

$$
C^{n}:=\underset{p+q=n}{\oplus} C^{p, q}
$$

com operador fronteira

$$
d+\delta: C^{n} \longrightarrow C^{n+1}
$$

e a cohomologia

$$
H^{*}(C, d+\delta)
$$

Como estaremos trabalhando com os inteiros, podemos ver um elemento $C^{n}$ através do reticulado, isto é, por exemplo, o reticulado de $C^{4}$ é

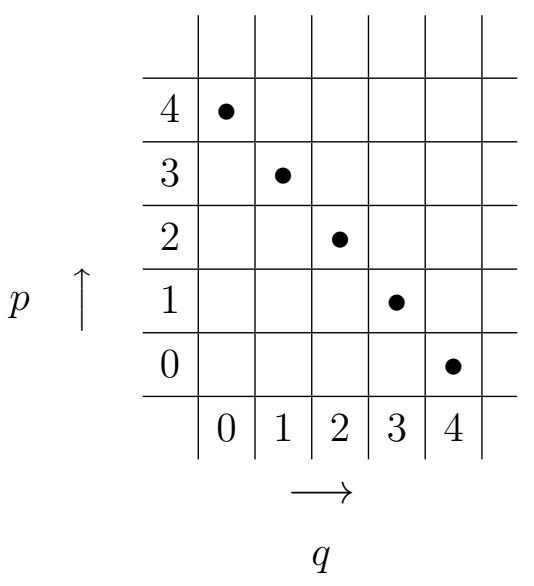

Agora, definindo

$$
C_{k}^{n}:=\bigoplus_{p+q=n, p \geq k} C^{p, q},
$$

temos que $C_{k}^{n}$ consiste dos elementos de $C^{n}$ que estão mais a direita da linha "vertical"que representa $k$. Observe o exemplo de $C_{2}^{4}$ :

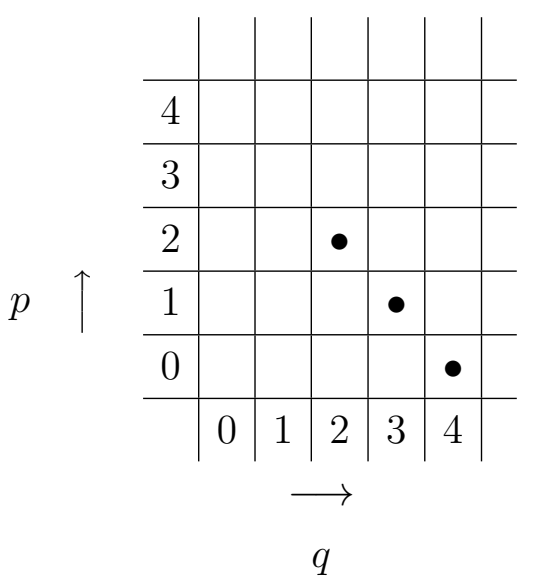


Considere agora os conjuntos

$$
Z_{k}^{n}:=\left\{z \in C_{k}^{n} \mid(d+\delta) z=0\right\}, B_{k}^{n}:=(d+\delta) C^{n-1} .
$$

A projeção

$$
Z_{k}^{n} \rightarrow Z_{k}^{n} /\left(B^{n} \cap Z_{k}^{n}\right)=H_{k}^{n}
$$

gera uma filtração decrescente

$$
\cdots \supset H_{k-1}^{n} \supset H_{k}^{n} \supset H_{k+1}^{n} \supset \cdots
$$

do grupo de cohomologia $H^{n}(C, d+\delta)$, isto é, denotando

$$
H^{k, n=k}:=H_{k}^{n} / H_{k+1}^{n},
$$

temos

$$
\operatorname{gr} H^{n}:=\underset{k}{\oplus} H^{k, n-k} .
$$

A sequência espectral que descreveremos é um esquema para calcular os quocientes a partir dos complexos "verticais" $\left(C^{r \cdot *}, d\right)$. E, para fazer isto, a ideia é construir uma sequência de complexos $\left(E_{r}, \delta_{r}\right)$ tal que $E_{r+1}$ é a cohomolodia do grau anterior, isto é a cohomologia de $\left(E_{r}, \delta_{r}\right)$.

Todo termo em $C^{n}$ se encontra na antidiagonal $\underset{i+j=n}{\oplus} C^{i, j}$. Chamaremos um elemento de termo líder se ele está na posição $(p, q)$ onde $p$ é o menor $i$ onde o componente é não vazio. Denotando por $Z^{p, q}$ os componentes do cociclo na posição $(p, q)$, temos que, dado $a \in C^{p, q}, a \in Z^{p, q}$ se o sistema de equações

$$
\begin{gathered}
d a=0 \\
\delta a=-d a_{1} \\
\delta a_{1}=-d a_{2} \\
\delta a_{2}=-d a_{3}
\end{gathered}
$$

admite solução

$$
\left(a_{1}, a_{2}, \cdots\right) \text { onde } a_{i} \in C^{p+i, q-i} \text {. }
$$

Ou seja, podemos "aumentar" $a$ por uma sequência de zig-zags através da $\mathbf{l}:=(i, j) \mid i+j=p+q\}$. Nos exemplos desta dissertação,

$$
C^{i, j}=0, \text { quando }|i-j|>m_{\mathbf{l}}
$$

para algum $m_{\mathbf{l}}$, lembrando que $i+j=p+q$. Desta forma, o sistema de equações D.1.3 terá solução para todo $i$ se ele tiver solução para um intervalo limitado de $i$. 
Um elemento

$$
z:=a \oplus a_{1} \oplus a_{2} \oplus \cdots,
$$

onde $a, a_{1}, \cdots$ são os elementos que satisfazem o sistema D.1.3, é dito pertencer a $Z_{p}^{n}$ e têm como coeficiente lider $a$.

Definimos agora $B^{p, q}$ como os termos $b$ que tem a propriedade que o sistema de equações

$$
\begin{gathered}
d c_{0}+\delta c_{-1}=b \\
d c_{-1}+\delta c_{-2}=0 \\
d c_{-2}+\delta c_{-3}=0
\end{gathered}
$$

admite uma solução

$$
\left(c_{0}, c_{-1}, c_{-2}, \cdots\right) \text { com } c_{-i} \in C^{p-i \cdot q+i-1} .
$$

Repare que se $b \in B^{p, q}$ então $b \in Z^{p, q}$. E, novamente, caso $C^{i, j}=0$ se $|i-j|>m_{\mathbf{l}}$, é suficiente mostrar o resultado para um intervalo limitado de $i$. Além disso, é fácil ver que o quociente $H^{p, q}$ em D.1.1 também pode ser escrito como

$$
H^{p, q}=Z^{p, q} / B^{p, q} .
$$

Calculemos então este quociente resolvendo o sistema D.1.3 indutivamente.

Defina $Z_{r}^{p, q} \subset C^{p, q}$ tal que $a \in Z_{r}^{p, q}$ se as primeiras $r-1$ equações de D.1.3 podem ser satisfeitas. Isto é, $a \in Z_{r}^{p, q}$ se e somente se, ele pode ser unido por uma sequência de zig-zags a um elemento $a_{r-1} \in C^{p_{r}, q_{r}}$ onde

$$
\left(p_{r}, q_{r}\right)=(p+r-1, q-r+1)
$$

é um ponto de $r-1$ unidades abaixo na diagonal $\mathbf{l}$ de $(p, q)$.

Vale observar que $Z^{p, q} \subset Z_{r}^{p, q}$ e $\lim _{r \rightarrow \infty} Z_{r}^{p, q}=Z^{p, q}$.

A questão agora é saber quando $a \in Z_{r}^{p, q}$ pode ser unido por uma sequência de zig-zags a um elemento em $C^{p_{r}+1, q_{r}-1}$. Estender a sequência pode não ser imediata, já que talvez precisamos alterar a solução parcial $\left(a_{1}, \cdots, a_{r-1}\right)$ por uma outra $\left(a_{1}^{\prime}, \cdots, a_{r-1}^{\prime}\right)$ de forma que a diferença entre as duas soluções estejam na fronteira, isto é, dado $a_{i}^{\star}=a_{i}^{\prime}-a_{i}$, temos que 


$$
\begin{gathered}
d a_{1}^{\star}=0 \\
\delta a_{1}^{\star}+d a_{2}^{\star}=0 \\
\vdots \\
\delta a_{r-2}^{\star}+d a_{r-1}^{\star}=0
\end{gathered}
$$

e, para darmos mais um passo no zig-zag, precisamos de um $a_{r}^{\star} \in C^{p_{r}+1, q_{r}-1}$ tal que

$$
\delta a_{r-1}=-\delta a_{r-1}^{\star}-d a_{r}^{\star}
$$

pois $\delta a_{r-1}^{\star}=\delta a_{r-1}^{\prime}-a_{r-1}$ e, portanto,

$$
\delta a_{r-1}=-d a_{r}^{\star}
$$

Defina então

$$
b:=\delta a_{r-1}, c_{0}:=-a_{r}^{\star}, c_{-i}:=-a_{r-1}^{\star}, i=1, \cdots, r-1
$$

e $c_{-i}=0$ para $i \geq r$. Assim, defina o conjunto

$$
B_{r}^{p, q} \subset B^{p, q}
$$

de forma que $b \in B_{r}^{p, q}$ se existe uma solução para D.1.5 $\operatorname{com} c_{-i}=0$ para $i \geq r$. Vale a observação de que

$$
\lim _{r \rightarrow \infty} B_{r}^{p, q}=B^{p, q}
$$

e que

$$
B_{1}^{p, q} \subset \cdots \subset B_{r}^{p, q} \subset \cdots \subset B^{p, q} \subset Z^{p, q} \subset \cdots Z_{r}^{p, q} \subset \cdots \subset Z_{1}^{p, q} .
$$

Assim, segue por construção que:

Teorema D.1.1 Seja $a \in Z_{r+1}^{p, q}$. Então

$$
a \in Z_{r+1}^{p, q} \Longleftrightarrow \delta a_{r-1} \in B^{p_{r}+1, q_{r}}
$$

para cada solução $\left(a_{1}, \cdots, a_{r-1}\right)$ das primeiras $r-1$ equações de D.1.3.

Dizemos que segue da definição pois se $a \in Z_{r+1}^{p, q}$ então temos, pela construção feita, $\delta a_{r-1}=-\delta a_{r-1}^{\star}-d a_{r}^{\star}$ e, portanto, $\left(a_{r-1}^{\star}, a_{r-2}^{\star}, \cdots, a_{1}^{\star}, 0, \cdots\right)$ satisfaz a condição de $\delta a_{r-1} \in B^{p_{r}+1, q_{r}}$. A volta da implicação é literalmente a volta passo a passo da construção.

Repare agora que $\delta a_{r-1}$ satisfaz o sistema de equações 


$$
\begin{gathered}
\delta a_{r-1}=: b \\
\delta a_{r-2}+d a_{r-1}=0 \\
\vdots \\
d a=0
\end{gathered}
$$

e, portanto, $\delta a_{r-1} \in B^{p_{r}+1, q_{r}}$. Na verdade, qualquer elemento em $B^{p_{r}+1, q_{r}}$ pode ser escrito como uma soma da forma

$$
\delta a_{r-1}+d c
$$

onde $c \in C^{p_{r}+1, q_{r}-1}$ e $\left(a, a_{1}, \cdots, a_{r-1}\right)$ é solução das $r-1$ primeiras equações de D.1.3. Podemos concluir que:

1. Defina

$$
E_{r}^{p, q}:=Z_{r}^{p, q} / B_{r}^{p, q}
$$

Como

$$
\delta a_{r-1} \in B_{r+1}^{p_{r}+1, q_{r}} \subset Z^{p_{r}+1, q_{r}} \subset Z_{r}^{p_{r}+1, q_{r}},
$$

podemos ver que $\delta a_{r-1}$ projeta-se sobre um elemento

$$
\delta_{r} a \in E_{r}^{p_{r}+1, q_{r}},
$$

isto é, como $\delta a_{r-1} \in B_{r+1}^{p_{r}+1, q_{r}}$, temos que existe solução parcial para as $r$ primeiras equações de D.1.5 e $c_{-i}=0$ para $i \geq r+1$, portanto, solução $\left(c_{0}, c_{-1}, \cdots, c_{-r+1}, 0, \cdots\right)$. Assim, podemos ver $\delta_{r} a \in E_{r}^{p_{r}+1, q_{r}}$ tendo como imagem a classe de $c_{-r+1}$ em $E^{p_{r}+1, q_{r}}=Z_{r}^{p, q} / B_{r}^{p, q}$ módulo $B^{p, q}$ pela escolha da solução parcial.

Com isto, podemos modificar o teorema D.1.1 e dizer que $a \in Z_{r}^{p, q}$ está em $Z_{r+1}^{p, q}$ se e somente se $\delta_{r} a=0$.

2. O elemento $\delta_{r} a$ depende apenas da classe de a módulo $B^{p, q}$. Assim, como $B_{r}^{p, q} \subset B^{p, q}$, podemos considerar $\delta_{r}$ como um mapa entre $E_{r}^{p, q}$ e $E^{p+r, q-r+1}$, isto é,

$$
\delta_{r}: E_{r}^{p, q} \rightarrow E_{r}^{p+r, q-r+1} .
$$

Observe o reticulado e os mapas abaixo: 


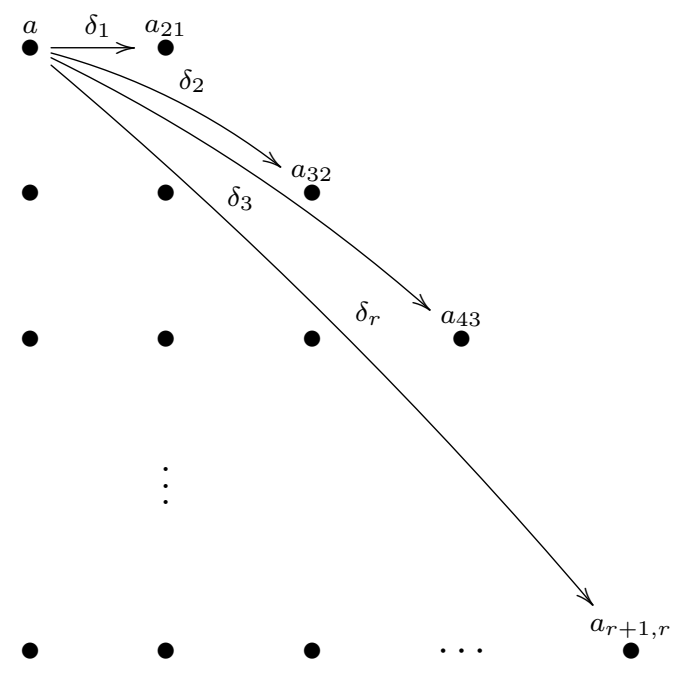

Sem considerar as classes precisamente, os mapas no reticulado acima, nos dá uma ideia geométrica de como se comporta o mapa $\delta_{r}$ a menos de $B^{p, q}$.

3. Pela equação D.1.10, a imagem do mapa $\delta_{r}$ é a projeção de $B_{r+1}^{p+r, q-r+1} \mathrm{em}$ $E_{r}^{p+r, q-r+1}$, assim, pelo teorema D.1.1, o núcleo deste mapa é a projeção de $Z_{r+1}^{p, q}$ em $E_{r}^{p, q}$. Portanto a sequência

$$
\cdots \stackrel{\delta_{r}}{\rightarrow} E_{r}^{p, q} \stackrel{\delta_{r}}{\rightarrow} \cdots
$$

é um complexo de cadeias, isto é, $\operatorname{Im} \delta_{r} \subset \operatorname{ker} \delta_{r}$. Portanto

$$
\left(\operatorname{ker} \delta_{r}\right) /\left(\operatorname{Im} \delta_{r}\right)=E_{r+1}^{p, q}
$$

na posição $(p, q)$.

Logo, conseguimos a propriedade que queríamos,

$$
H\left(E_{r}, \delta_{r}\right)=E_{r+1}
$$

para $r=1,2, \cdots$.

Repare que, por construção, $\delta_{r}$ tem bigrau $(r,-(r-1))$. Assim, se

$$
C^{i, j}=0 \text { se }|i-j|<m_{1}
$$

para algum $m_{\mathbf{l}}$ para cada diagonal $\mathbf{l}=\{(i, j) \mid i+j=p+q\}$, a sequência espectral se estabiliza

$$
E_{r}^{p, q}=E_{r+1}^{p, q}=\cdots
$$

para $r$ suficientemente grande (dependendo de $p$ e $q$ ). Assim, por D.1.6, temos finalmente o cálculo da graduação, isto é, 


$$
E_{\infty}^{p, q}=\lim _{r \rightarrow \infty} E_{r}^{p, q}=H^{p, q} .
$$

No caso $r=1, \delta_{1}$ está apenas transladando de coluna no reticulado, desta forma, podemos ver que

$$
E_{1}^{p, q}=H^{q}\left(C^{p, *}, d\right)
$$

é a cohomologia vertical de cada coluna. Assim, como $d$ e $\delta$ comutam, $\delta$ induz um mapa em cohomologia

$$
H^{q}\left(C^{p, *}, d\right) \rightarrow H^{q}\left(C^{p+1, *}, d\right)
$$

que é precisamente o mapa $\delta_{1}$. Desta forma, descrevemos $\left(E_{1}, \delta_{1}\right)$ e, portanto, $E_{2}$. Não entraremos muito no estudo, mas $\delta_{r}$ para $r \geq 2$ pode ser bem mais complicado.

\section{D.2}

\section{O Funtor Graduação}

Seja $(C, d, \delta)$ e $\left(C^{\prime}, d^{\prime}, \delta^{\prime}\right)$. Quando trabalhavamos com morfismos na categoria de $G^{*}$-módulos, exigiamos que que ele comutasse com as derivações para preservar certas propriedades, o mesmo acontecerá aqui. Consideremos primeiro

$$
\rho: C \rightarrow C^{\prime}
$$

morfismo quanto espaço vetoriais. Suponha então que $\rho$ seja um morfismo de complexo duplo de grau $(m, n)$. Isto quer dizer que se $a \in C^{i, j}$, então $\rho(a) \in C^{i+m, j+n}$. Suponha, por fim, que

$$
\rho d=d^{\prime} \rho
$$

e

$$
\rho \delta=\delta^{\prime} \rho
$$

Estas hipóteses garantem que o mapa

$$
\rho:(C, d+\delta) \rightarrow\left(C^{\prime}, d^{\prime}+\delta^{\prime}\right)
$$

é um mapa cocadeia de grau $m+n$ pois comuta com o operador fronteira. Desta forma, ele induz um mapa $\rho^{\star}$ na cohomologia,

$$
\rho^{\star}: H(C, d+\delta) \rightarrow H\left(C^{\prime}, d^{\prime}+\delta^{\prime}\right)
$$

de grau $m+n$. 
De forma análoga, fixando a linha, o mapa

$$
\rho:\left(C^{p, *}, d\right) \rightarrow\left(\left(C^{\prime}\right)^{p+m, *}, d^{\prime}\right)
$$

também é um mapa cocadeia e, portanto, $\rho$ define mapas pelo complexo de cocadeias. Logo, ele induz um mapa $\rho_{1}$ na cohomologia, isto é,

$$
\rho_{1}: E_{1} \rightarrow\left(E^{\prime}\right)_{1}
$$

pois $E_{1}^{p, q}=H^{q}\left(C^{p, *}, d\right)$. Em particula, $\rho_{1}$ tem grau $(m, n)$ e, como

$$
\rho \delta=\delta^{\prime} \rho
$$

temos que

$$
\rho_{1} \delta_{1}=\left(\delta^{\prime}\right)_{1} \rho
$$

Assim, indutivamente, obtemos o mapa

$$
\rho_{r}:\left(E_{r}, \delta_{r}\right) \rightarrow\left(\left(E^{\prime}\right)_{r},\left(\delta^{\prime}\right)_{r}\right)
$$

em que o mapa $\rho_{r}$ é um mapa induzido em cohomologia por $\rho_{r-1}$, já que, $E_{r}=H\left(E_{r}, \delta_{r}\right)$

Logo, como $H^{p, q}=Z^{p, q} / B^{p, q}$ e $E^{p, q}=Z_{r}^{p, q} / B_{r}^{p, q}$, temos que:

Teorema D.2.1 Se as duas sequências espectrais convergem, então

$$
\lim _{r \rightarrow \infty} \rho_{r}=g r \rho^{\star}
$$

E, como consequência,

Teorema D.2.2 Se $\rho_{r}$ é um isomorfismo para algum $r_{0}$, por construção, ele é um isomorfismo $r>r_{0}$. Logo, se as sequências espectrais convergem, $\rho^{\star}$ é um isomorfismo.

Uma aplicação imediata deste resultado é o seguinte corolário:

Corolário D.2.3 Se $E_{r}^{p, q}=0$ quando $p+q$ é ímpar, então a sequência espectral colapsa no estágio $E_{r}$, isto é, $E_{r}=E_{r+1}=\cdots$

Prova: De fato, o mapa $\delta_{r}: E_{r}^{p, q} \rightarrow E_{r}^{p+r, q-r+1}$ claramente muda a paridade de $p+q$. Logo, o domínio ou a imagem são 0 . Segue então que $\delta_{r} \equiv 0$ como queríamos. 


\section{Referências bibliográficas}

[BT] BOTT, R.; TU, L. W.. Differential forms in algebraic topology, volumen 82. Springer Science \& Business Media, 2013.

[DK] DUISTERMAAT, J. J.; KOLK, J. A.. Lie groups. Springer Science \& Business Media, 2012.

[GS] GUILLEMIN, V. W.; STERNBERG, S.. Supersymmetry and equivariant de Rham theory. Springer Science \& Business Media, 2013.

[Tu] TU, L. W.. An introduction to manifolds. second, 2011.

[Car] CARTAN, H.. Notion d'algebre différentielle; application aux groupes de lie et aux variétés ou opere un groupe de lie and la trangression dans un groupe de lie et dans un espace fibré principal. In: COLLOQUE DE TOPOLOGIE (BRUXELLES 1950), PARIS, MASSON, 1951.

[Chev] CHEVALLEY, C.. Invariants of finite groups generated by reflections. American Journal of Mathematics, 77(4):778-782, 1955.

[GS2] GUILLEMIN, V.; STERNBERG, S.. Symplectic techniques in physics. Cambridge university press, 1984.

[Hatc] HATCHER, A.. Algebraic topology. 2001. url: http://www. math. cornell. edu/ hatcher/at/at.pdf. AT. pdf, 2001.

[Hel] HELGASON, S.. Differential geometry and symmetric spaces, volumen 12. Academic Press, 1962.

[Hus] HUSEMOLLER, D.. Fiber bundles - 3rd ed. Springer-Verlag, 1993.

[Lee] LEE, J. M.. Smooth manifolds. In: INTRODUCTION TO SMOOTH MANIFOLDS, p. 1-31. Springer, 2013.

[Mar] MARTIN, L. A.. Grupos de Lie. Editora da Unicamp, 2016.

[War] WARNER, F. W.. Foundations of differentiable manifolds and Lie groups, volumen 94. Springer Science \& Business Media, 2013. 
[Zhe] ZHELOBENKO, D. P.. Compact Lie groups and their representations, volumen 40. American Mathematical Soc., 1973. 